\title{
Almost all short intervals containing prime numbers
}

\author{
by
}

ChaOhua Jia (Beijing)

1. Introduction. In 1937, Cramér [1] conjectured that every interval $\left(n, n+f(n) \log ^{2} n\right)$ contains a prime for some $f(n) \rightarrow 1$ as $n \rightarrow \infty$.

In 1943, assuming the Riemann Hypothesis, Selberg [19] showed that, for almost all $n$, the interval $\left(n, n+f(n) \log ^{2} n\right)$ contains a prime providing $f(n) \rightarrow \infty$ as $n \rightarrow \infty$. In the same paper, he also showed that, for almost all $n$, the interval $\left(n, n+n^{\frac{19}{77}+\varepsilon}\right)$ contains a prime.

In 1971, Montgomery [16] improved the exponent $\frac{19}{77}$ to $\frac{1}{5}$. The zero density estimate of Huxley [7] gives the exponent $\frac{1}{6}$.

In 1982, Harman [3] used the sieve method to prove that, for almost all $n$, the interval $\left(n, n+n^{\frac{1}{10}+\varepsilon}\right)$ contains a prime. Heath-Brown [5] and Harman [4] mentioned that the exponent $\frac{1}{12}$ can be achieved.

In [11], Jia Chaohua investigated the problem of the exceptional set of Goldbach numbers in the short interval. As a by-product, he proved that, for almost all $n$, the interval $\left(n, n+n^{\frac{1}{13}+\varepsilon}\right)$ contains prime numbers. Li Hongze [14] improved the exponent $\frac{1}{13}$ to $\frac{2}{27}$.

Recently, Jia Chaohua [12] showed that, for almost all $n$, the interval $\left(n, n+n^{\frac{1}{14}+\varepsilon}\right)$ contains prime numbers. Watt [20] also obtained the same result. Their methods are different. In [12] only classical methods are used and in [20] a new mean value estimate of Watt [21] is used in addition. $\mathrm{Li}$ Hongze [15] combined these methods to improve the exponent $\frac{1}{14}$ to $\frac{1}{15}$.

In this paper, we prove the following:

TheOREM. Suppose that $B$ is a sufficiently large positive constant, $\varepsilon$ is a sufficiently small positive constant and $X$ is sufficiently large. Then for positive integers $n \in(X, 2 X)$, except for $O\left(X \log ^{-B} X\right)$ values, the interval $\left(n, n+n^{\frac{1}{20}+\varepsilon}\right)$ contains at least $0.005 n^{\frac{1}{20}+\varepsilon} \log ^{-1} n$ prime numbers.

Project supported by the Tian Yuan Item in the National Natural Science Foundation of China. 
We apply a mean value estimate of Deshouillers and Iwaniec [2] (see Lemma 2). Using the classical mean value estimate instead of that of Deshouillers and Iwaniec, we can get the exponent $\frac{1}{18}$. We refer to [13] and the explanation in [11].

Throughout this paper, we always suppose that $B$ is a sufficiently large positive constant, $\varepsilon$ is a sufficiently small positive constant and $\varepsilon_{1}=\varepsilon^{2}$, $\delta=\varepsilon^{\frac{1}{3}}$. We also suppose that $X$ is sufficiently large and that $x \in(X, 2 X)$, $\eta=\frac{1}{2} X^{-\frac{19}{20}+\varepsilon}$. Let $c, c_{1}$ and $c_{2}$ denote positive constants which have different values at different places. $m \sim M$ means that there are positive constants $c_{1}$ and $c_{2}$ such that $c_{1} M<m \leq c_{2} M$. We often use $M(s)$ ( $M$ may be another capital letter) to denote a Dirichlet polynomial of the form

$$
M(s)=\sum_{m \sim M} \frac{a(m)}{m^{s}}
$$

where $a(m)$ is a complex number with $a(m)=O(1)$.

The author would like to thank Profs. Wang Yuan and Pan Chengbiao for their encouragement.

\section{Mean value estimate (I)}

Lemma 1. Suppose that $X^{\delta} \ll H \ll X^{\frac{9}{85}}, M H=X, M(s)$ is a Dirichlet polynomial and

$$
H(s)=\sum_{h \sim H} \frac{\Lambda(h)}{h^{s}} .
$$

Let $b=1+1 / \log X, T_{0}=\log ^{\frac{B}{\varepsilon}} X$. Then for $T_{0} \leq T \leq X$, we have

$$
\min ^{2}\left(\eta, \frac{1}{T}\right) \int_{T}^{2 T}|M(b+i t) H(b+i t)|^{2} d t \ll \eta^{2} \log ^{-10 B} x
$$

Proof. Let $s=b+i t$. By the zero-free region of the $\zeta$ function, for $|t| \leq 2 X$ we have

$$
\sum_{c_{1} H<h \leq c_{2} H} \frac{\Lambda(h)}{h^{s}}=\frac{\left(c_{2} H\right)^{1-s}-\left(c_{1} H\right)^{1-s}}{1-s}+O\left(\log ^{-\frac{2 B}{\varepsilon}} x\right) .
$$

So, for $T_{0} \leq|t| \leq 2 X$,

$$
H(s) \ll \log ^{-\frac{B}{\varepsilon}} x .
$$

According to the discussion in [6], there are $O\left(\log ^{2} X\right)$ sets $S(V, W)$, where $S(V, W)$ is the set of $t_{k}(k=1, \ldots, K)$ with the property $\left|t_{r}-t_{s}\right| \geq 1$ $(r \neq s)$. Moreover,

$$
V \leq M^{\frac{1}{2}}\left|M\left(b+i t_{k}\right)\right|<2 V, \quad W \leq H^{\frac{1}{2}}\left|H\left(b+i t_{k}\right)\right|<2 W,
$$


where $X^{-1} \leq M^{-\frac{1}{2}} V, X^{-1} \leq H^{-\frac{1}{2}} W$ and $V \ll M^{\frac{1}{2}}, W \ll H^{\frac{1}{2}} \log ^{-\frac{B}{\varepsilon}} x$. Then

(3) $\int_{T}^{2 T}|M(b+i t) H(b+i t)|^{2} d t \ll V^{2} W^{2} x^{-1} \log ^{2} x|S(V, W)|+O\left(x^{-2 \varepsilon_{1}}\right)$,

where $S(V, W)$ is one of sets with the above properties.

Assume $X^{\frac{1}{k+1}} \leq H<X^{\frac{1}{k}}$, where $k$ is a positive integer, $k \geq 9$ and $k \delta \ll 1$. Applying the mean value estimate (see Section 3 of [9] or Lemma 7 of [11]) to $M(s)$ and $H^{k}(s)$, we have

$$
\begin{aligned}
& |S(V, W)| \ll V^{-2}(M+T) \log ^{d} x, \\
& |S(V, W)| \ll W^{-2 k}\left(H^{k}+T\right) \log ^{d} x,
\end{aligned}
$$

where $d=c / \delta^{2}$. Applying the Halász method (see Section 3 of [9] or Lemma 7 of [11]) to $M(s)$ and $H^{k}(s)$, we have

$$
\begin{aligned}
& |S(V, W)| \ll\left(V^{-2} M+V^{-6} M T\right) \log ^{d} x, \\
& |S(V, W)| \ll\left(W^{-2 k} H^{k}+W^{-6 k} H^{k} T\right) \log ^{d} x .
\end{aligned}
$$

Thus,

where

$$
V^{2} W^{2}|S(V, W)| \ll V^{2} W^{2} F \log ^{d} x
$$

$$
\begin{array}{r}
F=\min \left\{V^{-2}(M+T), V^{-2} M+V^{-6} M T, W^{-2 k}\left(H^{k}+T\right),\right. \\
\left.W^{-2 k} H^{k}+W^{-6 k} H^{k} T\right\} .
\end{array}
$$

It will be proved that

$$
\min ^{2}\left(\eta, \frac{1}{T}\right) V^{2} W^{2} F \ll \eta^{2} x \log ^{-\frac{B}{\varepsilon}} x .
$$

We consider four cases.

(a) $F \leq 2 V^{-2} M, 2 W^{-2 k} H^{k}$. Then

$$
\begin{aligned}
V^{2} W^{2} F & \ll V^{2} W^{2} \min \left\{V^{-2} M, W^{-2 k} H^{k}\right\} \\
& \leq V^{2} W^{2}\left(V^{-2} M\right)^{1-\frac{1}{2 k}}\left(W^{-2 k} H^{k}\right)^{\frac{1}{2 k}} \\
& =V^{\frac{1}{k}} W M^{1-\frac{1}{2 k}} H^{\frac{1}{2}} \ll x \log ^{-\frac{B}{\varepsilon}} x
\end{aligned}
$$

and so

$$
\min ^{2}\left(\eta, \frac{1}{T}\right) V^{2} W^{2} F \ll \eta^{2} x \log ^{-\frac{B}{\varepsilon}} x .
$$

(b) $F>2 V^{-2} M, 2 W^{-2 k} H^{k}$. Then

$$
\begin{aligned}
V^{2} W^{2} F & \ll V^{2} W^{2} \min \left\{V^{-2} T, V^{-6} M T, W^{-2 k} T, W^{-6 k} H^{k} T\right\} \\
& \leq V^{2} W^{2}\left(V^{-2}\right)^{1-\frac{3}{2 k}}\left(V^{-6} M\right)^{\frac{1}{2 k}}\left(W^{-2 k}\right)^{\frac{1}{k}} T=M^{\frac{1}{2 k}} T .
\end{aligned}
$$


Since $k \geq 9$, we have $H \geq X^{\frac{1}{k+1}} \geq X^{1-\frac{k}{10}}, M^{\frac{1}{2 k}} \ll X^{\frac{1}{20}}$, and so

$$
\min ^{2}\left(\eta, \frac{1}{T}\right) V^{2} W^{2} F \ll \frac{\eta}{T} x^{\frac{1}{20}} T \ll \eta^{2} x^{1-\varepsilon_{1}} .
$$

(c) $F \leq 2 V^{-2} M, F>2 W^{-2 k} H^{k}$. Then

$$
\begin{aligned}
V^{2} W^{2} F & \ll V^{2} W^{2} \min \left\{V^{-2} M, W^{-2 k} T, W^{-6 k} H^{k} T\right\} \\
& \leq V^{2} W^{2}\left(V^{-2} M\right)^{1-\frac{1}{3 k}}\left(W^{-6 k} H^{k} T\right)^{\frac{1}{3 k}} \\
& \ll M H^{\frac{1}{3}} T^{\frac{1}{3 k}},
\end{aligned}
$$

since $V \ll M^{\frac{1}{2}}$. As $H \geq X^{\frac{1}{k+1}} \geq X^{\frac{19}{20} \cdot \frac{1}{2 k}+\varepsilon}$, we have

$$
\min ^{2}\left(\eta, \frac{1}{T}\right) V^{2} W^{2} F \ll \eta^{2-\frac{1}{3 k}} T^{-\frac{1}{3 k}} x^{1-\frac{19}{20} \cdot \frac{1}{3 k}} T^{\frac{1}{3 k}} x^{-\varepsilon_{1}} \ll \eta^{2} x^{1-\varepsilon_{1}} .
$$

(d) $F>2 V^{-2} M, F \leq 2 W^{-2 k} H^{k}$. Then

$$
\begin{aligned}
V^{2} W^{2} F & \ll V^{2} W^{2} \min \left\{V^{-2} T, V^{-6} M T, W^{-2 k} H^{k}\right\} \\
& \leq V^{2} W^{2}\left(V^{-2} T\right)^{1-\frac{3}{2 k}}\left(V^{-6} M T\right)^{\frac{1}{2 k}}\left(W^{-2 k} H^{k}\right)^{\frac{1}{k}} \\
& =M^{\frac{1}{2 k}} H T^{1-\frac{1}{k}} .
\end{aligned}
$$

If $k \geq 10$, then $H \leq X^{\frac{1}{k}} \leq X^{1-\frac{19(k-1)}{10(2 k-1)}}, M \gg X^{\frac{19(k-1)}{10(2 k-1)}}$, and so

$$
\min ^{2}\left(\eta, \frac{1}{T}\right) V^{2} W^{2} F \ll \eta^{1+\frac{1}{k}} x^{1-\frac{19}{20}\left(1-\frac{1}{k}\right)} \ll \eta^{2} x^{1-\varepsilon_{1}} .
$$

If $k=9$, then $X^{\frac{1}{10}} \leq H \ll X^{\frac{9}{85}}, M \gg X^{\frac{76}{85}}$, and so

$$
\min ^{2}\left(\eta, \frac{1}{T}\right) V^{2} W^{2} F \ll \eta^{2}\left(x^{-\frac{19}{20}+\varepsilon}\right)^{-\frac{8}{9}} x^{1-\frac{38}{45}} \ll \eta^{2} x^{1-\varepsilon_{1}} .
$$

Combining the above, we obtain (4). Hence, Lemma 1 follows.

Lemma 2. Suppose that $N(s)$ is a Dirichlet polynomial and

$$
L(s)=\sum_{c_{1} L<l \leq c_{2} L} \frac{1}{l^{s}} .
$$

Let $T \geq 1$. Then

$$
\begin{aligned}
I & =\int_{T}^{2 T}\left|L\left(\frac{1}{2}+i t\right)\right|^{4}\left|N\left(\frac{1}{2}+i t\right)\right|^{2} d t \\
& \ll\left(T+N^{2} T^{\frac{1}{2}}+N^{\frac{5}{4}}\left(T \min \left(L, \frac{T}{L}\right)\right)^{\frac{1}{2}}+N L^{2} T^{-2}\right) T^{\varepsilon_{1}} .
\end{aligned}
$$


Proof. First we assume $c_{1} L \leq T^{\frac{1}{2}}$. If $N \leq T$, then by the discussion in Section 2 of [2], we have

$$
I \ll\left(T+N^{2} T^{\frac{1}{2}}+N^{\frac{5}{4}}(T L)^{\frac{1}{2}}\right) T^{\varepsilon_{1}} .
$$

If $N>T$, the mean value estimate yields

$$
I \ll\left(L^{2} N+T\right)(L N)^{\varepsilon_{1}} \ll\left(T+N^{2} T^{\frac{1}{2}}\right) T^{\varepsilon_{1}} .
$$

Now we assume $T^{\frac{1}{2}}<c_{1} L \leq 2 T$. By the discussion in Section 2 of [2], we get

$$
I \ll\left(T+N^{2} T^{\frac{1}{2}}+N^{\frac{5}{4}}\left(T \cdot \frac{T}{L}\right)^{\frac{1}{2}}\right) T^{\varepsilon_{1}} .
$$

Lastly we assume $2 T<c_{1} L$. It follows from Theorem 1 on page 442 of [18] that

$$
\sum_{c_{1} L<l \leq c_{2} L} \frac{1}{l^{\frac{1}{2}+i t}}=\frac{\left(c_{2} L\right)^{\frac{1}{2}-i t}-\left(c_{1} L\right)^{\frac{1}{2}-i t}}{\frac{1}{2}-i t}+O\left(\frac{1}{L^{\frac{1}{2}}}\right) .
$$

Hence,

$$
L\left(\frac{1}{2}+i t\right) \ll \frac{L^{\frac{1}{2}}}{|t|}+\frac{1}{L^{\frac{1}{2}}} \ll \frac{L^{\frac{1}{2}}}{|t|}
$$

and

$$
I \ll \frac{L^{2}}{T^{4}} \int_{T}^{2 T}\left|N\left(\frac{1}{2}+i t\right)\right|^{2} d t \ll \frac{L^{2}}{T^{4}}(N+T) \ll \frac{N L^{2}}{T^{2}} .
$$

Combining the above, we get Lemma 2 .

Lemma 3. Suppose that $M N L=X, M(s), N(s)$ are Dirichlet polynomials, and

$$
L(s)=\sum_{l \sim L} \frac{1}{l^{s}} .
$$

Let $b=1+1 / \log X, T_{1}=\sqrt{L}$. Assume further that $M$ and $N$ lie in one of the following regions:

$$
M \ll X^{\frac{9}{32}},
$$

$$
\begin{aligned}
& N \ll M^{\frac{2}{3}} X^{\frac{1}{10}} \\
& X^{\frac{9}{32}} \ll M \ll X^{\frac{53}{160}}, \quad N \ll X^{\frac{23}{80}} ; \\
& X^{\frac{53}{160}} \ll M \ll X^{\frac{13}{32}}, \quad N \ll M^{-\frac{2}{3}} X^{\frac{61}{120}} ; \\
& X^{\frac{13}{32}} \ll M \ll X^{\frac{321}{680}}, \quad N \ll M^{-2} X^{\frac{21}{20}} \text {. }
\end{aligned}
$$

Then for $T_{1} \leq T \leq X$, we have

$$
\min ^{2}\left(\eta, \frac{1}{T}\right) \int_{T}^{2 T}|M(b+i t) N(b+i t) L(b+i t)|^{2} d t \ll \eta^{2} x^{-2 \varepsilon_{1}} .
$$


Proof. It is sufficient to show that

$$
I=\min ^{2}\left(\eta, \frac{1}{T}\right) \int_{T}^{2 T}\left|M\left(\frac{1}{2}+i t\right) N\left(\frac{1}{2}+i t\right) L\left(\frac{1}{2}+i t\right)\right|^{2} d t \ll \eta^{2} x^{1-2 \varepsilon_{1}}
$$

We shall show that the above inequality holds, providing $M$ and $N$ satisfy the following conditions:

$$
\begin{gathered}
M^{2} N \ll X^{\frac{21}{20}}, \quad M^{2} N^{3} \ll X^{\frac{61}{40}}, \quad M^{6} N^{7} \ll X^{\frac{41}{10}}, \\
N \ll X^{\frac{23}{80}}, \quad N^{3} \ll M^{2} X^{\frac{3}{10}} .
\end{gathered}
$$

Using the mean value estimate and Lemma 2, we have

$$
\begin{aligned}
\int_{T}^{2 T} \mid M\left(\frac{1}{2}+i t\right) & \left.N\left(\frac{1}{2}+i t\right) L\left(\frac{1}{2}+i t\right)\right|^{2} d t \\
\ll & \left(\int_{T}^{2 T}\left|M\left(\frac{1}{2}+i t\right)\right|^{4}\left|N\left(\frac{1}{2}+i t\right)\right|^{2} d t\right)^{\frac{1}{2}} \\
& \times\left(\int_{T}^{2 T}\left|L\left(\frac{1}{2}+i t\right)\right|^{4}\left|N\left(\frac{1}{2}+i t\right)\right|^{2} d t\right)^{\frac{1}{2}} \\
\ll & \left(M^{2} N+T\right)^{\frac{1}{2}}\left(T+N^{2} T^{\frac{1}{2}}+N^{\frac{5}{4}}(T L)^{\frac{1}{2}}+N L^{2} T^{-2}\right)^{\frac{1}{2}} T^{\varepsilon_{1}} .
\end{aligned}
$$

Hence,

$$
\begin{aligned}
I & \ll \min ^{2}\left(\eta, \frac{1}{T}\right)\left(M^{2} N+T\right)^{\frac{1}{2}}\left(T+N^{2} T^{\frac{1}{2}}+N^{\frac{5}{4}}(T L)^{\frac{1}{2}}\right)^{\frac{1}{2}} T^{\varepsilon_{1}}+\eta^{2} T_{1}^{-\frac{1}{2}} x^{1+\varepsilon_{1}} \\
& \ll \eta^{2}\left(M^{2} N+\eta^{-1}\right)^{\frac{1}{2}}\left(\eta^{-1}+N^{2} \eta^{-\frac{1}{2}}+N^{\frac{5}{4}}\left(\eta^{-1} L\right)^{\frac{1}{2}}\right)^{\frac{1}{2}} x^{\varepsilon_{1}}+\eta^{2} x^{1-2 \varepsilon_{1}} \\
& \ll \eta^{2} x^{1-2 \varepsilon_{1}} .
\end{aligned}
$$

In every region of (5), our conditions are satisfied. So Lemma 3 follows.

Lemma 4. Under the assumptions of Lemma 3, (5) being replaced by the region

$$
M \ll X^{\frac{21}{40}}, \quad N \ll X^{\frac{19}{160}},
$$

for $T_{1} \leq T \leq X$, we have

$$
\min ^{2}\left(\eta, \frac{1}{T}\right) \int_{T}^{2 T}|M(b+i t) N(b+i t) L(b+i t)|^{2} d t \ll \eta^{2} x^{-2 \varepsilon_{1}} .
$$

Proof. It is sufficient to show that

$$
I=\min ^{2}\left(\eta, \frac{1}{T}\right) \int_{T}^{2 T}\left|M\left(\frac{1}{2}+i t\right) N\left(\frac{1}{2}+i t\right) L\left(\frac{1}{2}+i t\right)\right|^{2} d t \ll \eta^{2} x^{1-2 \varepsilon_{1}}
$$


Using the mean value estimate and Lemma 2, we have

$$
\begin{aligned}
I \ll & \min ^{2}\left(\eta, \frac{1}{T}\right)\left(\int_{T}^{2 T}\left|M\left(\frac{1}{2}+i t\right)\right|^{4} d t\right)^{\frac{1}{2}} \\
& \times\left(\int_{T}^{2 T}\left|L\left(\frac{1}{2}+i t\right)\right|^{4}\left|N\left(\frac{1}{2}+i t\right)\right|^{4} d t\right)^{\frac{1}{2}} \\
\ll & \min ^{2}\left(\eta, \frac{1}{T}\right)\left(M^{2}+T\right)^{\frac{1}{2}}\left(T+N^{4} T^{\frac{1}{2}}\right. \\
& \left.+N^{\frac{5}{2}}\left(T \min \left(L, \frac{T}{L}\right)\right)^{\frac{1}{2}}+N^{2} L^{2} T^{-2}\right)^{\frac{1}{2}} T^{\varepsilon_{1}} \\
\ll & \eta^{2}\left(M^{2}+\eta^{-1}\right)^{\frac{1}{2}}\left(\eta^{-1}+N^{4} \eta^{-\frac{1}{2}}\right)^{\frac{1}{2}} x^{\varepsilon_{1}}+\eta^{2} M N^{\frac{5}{4}}\left(\eta^{-1} L\right)^{\frac{1}{4}} x^{\varepsilon_{1}} \\
& +N^{\frac{5}{4}} \eta^{2-\frac{7}{8}} x^{\varepsilon_{1}}+\eta^{2} T_{1}^{-\frac{1}{2}} x^{1+\varepsilon_{1}} \\
\ll & \eta^{2} x^{1-2 \varepsilon_{1}},
\end{aligned}
$$

since $\min (L, T / L) \leq T^{\frac{1}{2}}$. Thus Lemma 4 follows.

\section{Mean value estimate (II)}

Lemma 5. Suppose that $M H K=X$ and $M(s), H(s)$ and $K(s)$ are Dirichlet polynomials, and $G(s)=M(s) H(s) K(s)$. Let $b=1+1 / \log X$, $T_{0}=\log ^{\frac{B}{\varepsilon}} X$. Assume further that for $T_{0} \leq|t| \leq 2 X, M(b+i t) \ll \log ^{-\frac{B}{\varepsilon}} x$ and $H(b+i t) \ll \log ^{-\frac{B}{\varepsilon}} x$. Moreover, suppose that $M$ and $H$ satisfy one of the following three conditions:

1) $M H \ll X^{\frac{157}{290}}, X^{\frac{19}{110}} \ll H, M^{29} / H \ll X^{10}, X^{\frac{3}{10}} \ll M, H^{29} / M \ll$ $X^{\frac{31}{5}}, X^{\frac{57}{10}} \ll M^{12} H^{11}$

2) $M H \ll X^{\frac{26}{45}}, M^{29} H^{19} \ll X^{\frac{59}{4}}, X^{\frac{38}{45}} \ll M^{2} H, M^{2} H^{11} \ll X^{\frac{29}{10}}$, $X^{\frac{57}{2}} \ll M^{58} H^{49}$

3) $M H \ll X^{\frac{25}{44}}, X^{\frac{19}{100}} \ll H, M^{6} H \ll X^{\frac{11}{5}}, X^{\frac{19}{70}} \ll M, M H^{8} \ll X^{\frac{23}{10}}$, $X^{\frac{57}{20}} \ll M^{6} H^{5}$.

Then for $T_{0} \leq T \leq X$, we have

$$
\min ^{2}\left(\eta, \frac{1}{T}\right) \int_{T}^{2 T}|G(b+i t)|^{2} d t \ll \eta^{2} \log ^{-10 B} x .
$$

Pr o of. Using the method of Lemma 1 , we only show that for $T=1 / \eta=$ $2 X^{\frac{19}{20}-\varepsilon}$,

$$
I=\int_{T}^{2 T}|G(b+i t)|^{2} d t \ll \log ^{-10 B} x .
$$


I. First, we assume condition 1). On applying the mean value estimate and Halász method to $M^{3}(s), H^{5}(s)$ and $K^{2}(s)$, we get

$$
I \ll U^{2} V^{2} W^{2} x^{-1} F \log ^{c} x,
$$

where

$$
\begin{gathered}
F=\min \left\{V^{-6}\left(M^{3}+T\right), V^{-6} M^{3}+V^{-18} M^{3} T, W^{-10}\left(H^{5}+T\right),\right. \\
\left.W^{-10} H^{5}+W^{-30} H^{5} T, U^{-4}\left(K^{2}+T\right), U^{-4} K^{2}+U^{-12} K^{2} T\right\} .
\end{gathered}
$$

We discuss the following cases:

(a) $F \leq 2 V^{-6} M^{3}, 2 W^{-10} H^{5}, 2 U^{-4} K^{2}$. Then

$$
\begin{aligned}
U^{2} V^{2} W^{2} F & \ll U^{2} V^{2} W^{2} \min \left\{V^{-6} M^{3}, W^{-10} H^{5}, U^{-4} K^{2}\right\} \\
& \leq U^{2} V^{2} W^{2}\left(V^{-6} M^{3}\right)^{\frac{3}{10}}\left(W^{-10} H^{5}\right)^{\frac{1}{5}}\left(U^{-4} K^{2}\right)^{\frac{1}{2}} \\
& =V^{\frac{1}{5}} M^{\frac{9}{10}} H K \ll x \log ^{-11 B} x .
\end{aligned}
$$

(b) $F \leq 2 V^{-6} M^{3}, 2 W^{-10} H^{5}, F>2 U^{-4} K^{2}$. Then

$$
\begin{aligned}
U^{2} V^{2} W^{2} F & \ll U^{2} V^{2} W^{2} \min \left\{V^{-6} M^{3}, W^{-10} H^{5}, U^{-4} T, U^{-12} K^{2} T\right\} \\
& \leq U^{2} V^{2} W^{2}\left(V^{-6} M^{3}\right)^{\frac{1}{3}}\left(W^{-10} H^{5}\right)^{\frac{1}{5}}\left(U^{-4} T\right)^{\frac{9}{20}}\left(U^{-12} K^{2} T\right)^{\frac{1}{60}} \\
& =T^{\frac{7}{15}} M H K^{\frac{1}{30}} \ll x^{1-\varepsilon_{1}} .
\end{aligned}
$$

(c) $F \leq 2 V^{-6} M^{3}, F>2 W^{-10} H^{5}, F \leq 2 U^{-4} K^{2}$. Then

$$
\begin{aligned}
U^{2} V^{2} W^{2} F & \ll U^{2} V^{2} W^{2} \min \left\{V^{-6} M^{3}, W^{-10} T, W^{-30} H^{5} T, U^{-4} K^{2}\right\} \\
& \leq U^{2} V^{2} W^{2}\left(V^{-6} M^{3}\right)^{\frac{1}{3}}\left(W^{-10} T\right)^{\frac{3}{20}}\left(W^{-30} H^{5} T\right)^{\frac{1}{60}}\left(U^{-4} K^{2}\right)^{\frac{1}{2}} \\
& =T^{\frac{1}{6}} M K H^{\frac{1}{12}} \ll x^{1-\varepsilon_{1}} .
\end{aligned}
$$

(d) $F \leq 2 V^{-6} M^{3}, F>2 W^{-10} H^{5}, 2 U^{-4} K^{2}$. Then

$$
\begin{aligned}
U^{2} & V^{2} W^{2} F \\
& \ll U^{2} V^{2} W^{2} \min \left\{V^{-6} M^{3}, W^{-10} T, W^{-30} H^{5} T, U^{-4} T, U^{-12} K^{2} T\right\} \\
& \leq U^{2} V^{2} W^{2}\left(V^{-6} M^{3}\right)^{\frac{1}{3}}\left(W^{-10} T\right)^{\frac{1}{5}}\left(U^{-4} T\right)^{\frac{9}{20}}\left(U^{-12} K^{2} T\right)^{\frac{1}{60}} \\
& =T^{\frac{2}{3}} M K^{\frac{1}{30}} \ll x^{1-\varepsilon_{1}} .
\end{aligned}
$$

(e) $F>2 V^{-6} M^{3}, F \leq 2 W^{-10} H^{5}, 2 U^{-4} K^{2}$. Then

$$
\begin{aligned}
U^{2} V^{2} W^{2} F & \ll U^{2} V^{2} W^{2} \min \left\{V^{-6} T, V^{-18} M^{3} T, W^{-10} H^{5}, U^{-4} K^{2}\right\} \\
& \leq U^{2} V^{2} W^{2}\left(V^{-6} T\right)^{\frac{17}{60}}\left(V^{-18} M^{3} T\right)^{\frac{1}{60}}\left(W^{-10} H^{5}\right)^{\frac{1}{5}}\left(U^{-4} K^{2}\right)^{\frac{1}{2}} \\
& =T^{\frac{3}{10}} M^{\frac{1}{20}} H K \ll x^{1-\varepsilon_{1}} .
\end{aligned}
$$


(f) $F>2 V^{-6} M^{3}, F \leq 2 W^{-10} H^{5}, F>2 U^{-4} K^{2}$. Then

$$
\begin{aligned}
U^{2} V^{2} & W^{2} F \\
& \ll U^{2} V^{2} W^{2} \min \left\{V^{-6} T, V^{-18} M^{3} T, W^{-10} H^{5}, U^{-4} T, U^{-12} K^{2} T\right\} \\
& \leq U^{2} V^{2} W^{2}\left(V^{-6} T\right)^{\frac{1}{3}}\left(W^{-10} H^{5}\right)^{\frac{1}{5}}\left(U^{-4} T\right)^{\frac{9}{20}}\left(U^{-12} K^{2} T\right)^{\frac{1}{60}} \\
& =T^{\frac{4}{5}} H K^{\frac{1}{30}} \ll x^{1-\varepsilon_{1}} .
\end{aligned}
$$

(g) $F>2 V^{-6} M^{3}, 2 W^{-10} H^{5}, F \leq 2 U^{-4} K^{2}$. Then

$$
\begin{aligned}
U^{2} V^{2} & W^{2} F \\
& \ll U^{2} V^{2} W^{2} \min \left\{V^{-6} T, V^{-18} M^{3} T, W^{-10} T, W^{-30} H^{5} T, U^{-4} K^{2}\right\} \\
& \leq U^{2} V^{2} W^{2}\left(V^{-6} T\right)^{\frac{1}{3}}\left(W^{-10} T\right)^{\frac{3}{20}}\left(W^{-30} H^{5} T\right)^{\frac{1}{60}}\left(U^{-4} K^{2}\right)^{\frac{1}{2}} \\
& =T^{\frac{1}{2}} H^{\frac{1}{12}} K \ll x^{1-\varepsilon_{1}} .
\end{aligned}
$$

(h) $F>2 V^{-6} M^{3}, 2 W^{-10} H^{5}, 2 U^{-4} K^{2}$. Then

$$
\begin{aligned}
& U^{2} V^{2} W^{2} F \\
& \quad \ll U^{2} V^{2} W^{2} \min \left\{V^{-6}, V^{-18} M^{3}, W^{-10}, W^{-30} H^{5}, U^{-4}, U^{-12} K^{2}\right\} T \\
& \quad \leq U^{2} V^{2} W^{2}\left(V^{-6}\right)^{\frac{17}{60}}\left(V^{-18} M^{3}\right)^{\frac{1}{60}}\left(W^{-10}\right)^{\frac{1}{5}}\left(U^{-4}\right)^{\frac{1}{2}} T \\
& \quad=T M^{\frac{1}{20}} \ll x^{1-\varepsilon_{1}},
\end{aligned}
$$

since $M \ll X$.

II. Next, we assume condition 2). On applying the mean value estimate and Halász method to $M^{2}(s) H(s), H^{5}(s)$ and $K^{2}(s)$, we get

$$
I \ll U^{2} V^{2} W^{2} x^{-1} F \log ^{c} x,
$$

where

$$
\begin{aligned}
F=\min \{ & V^{-4} W^{-2}\left(M^{2} H+T\right), V^{-4} W^{-2} M^{2} H+V^{-12} W^{-6} M^{2} H T, \\
& W^{-10}\left(H^{5}+T\right), W^{-10} H^{5}+W^{-30} H^{5} T, U^{-4}\left(K^{2}+T\right), \\
& \left.U^{-4} K^{2}+U^{-12} K^{2} T\right\} .
\end{aligned}
$$

We consider several cases:

(a) $F \leq 2 V^{-4} W^{-2} M^{2} H, 2 W^{-10} H^{5}, 2 U^{-4} K^{2}$. Then

$$
\begin{aligned}
U^{2} V^{2} W^{2} F & \ll U^{2} V^{2} W^{2} \min \left\{V^{-4} W^{-2} M^{2} H, W^{-10} H^{5}, U^{-4} K^{2}\right\} \\
& \leq U^{2} V^{2} W^{2}\left(V^{-4} W^{-2} M^{2} H\right)^{\frac{3}{8}}\left(W^{-10} H^{5}\right)^{\frac{1}{8}}\left(U^{-4} K^{2}\right)^{\frac{1}{2}} \\
& =V^{\frac{1}{2}} M^{\frac{3}{4}} H K \ll x \log ^{-11 B} x .
\end{aligned}
$$


(b) $F \leq 2 V^{-4} W^{-2} M^{2} H, 2 W^{-10} H^{5}, F>2 U^{-4} K^{2}$. Then

$$
\begin{aligned}
U^{2} V^{2} W^{2} F & \\
& \ll U^{2} V^{2} W^{2} \min \left\{V^{-4} W^{-2} M^{2} H, W^{-10} H^{5}, U^{-4} T, U^{-12} K^{2} T\right\} \\
& \leq U^{2} V^{2} W^{2}\left(V^{-4} W^{-2} M^{2} H\right)^{\frac{1}{2}}\left(W^{-10} H^{5}\right)^{\frac{1}{10}}\left(U^{-4} T\right)^{\frac{7}{20}}\left(U^{-12} K^{2} T\right)^{\frac{1}{20}} \\
& =T^{\frac{2}{5}} M H K^{\frac{1}{10}} \ll x^{1-\varepsilon_{1}} .
\end{aligned}
$$

(c) $F \leq 2 V^{-4} W^{-2} M^{2} H, F>2 W^{-10} H^{5}, F \leq 2 U^{-4} K^{2}$. Then

$$
\begin{aligned}
U^{2} V^{2} W^{2} F & \ll U^{2} V^{2} W^{2} \min \left\{V^{-4} W^{-2} M^{2} H, W^{-10} T, W^{-30} H^{5} T, U^{-4} K^{2}\right\} \\
& \leq U^{2} V^{2} W^{2}\left(V^{-4} W^{-2} M^{2} H\right)^{\frac{1}{2}}\left(U^{-4} K^{2}\right)^{\frac{1}{2}} \\
& =W H^{\frac{1}{2}} M K \ll x \log ^{-11 B} x .
\end{aligned}
$$

(d) $F \leq 2 V^{-4} W^{-2} M^{2} H, F>2 W^{-10} H^{5}, 2 U^{-4} K^{2}$. Then

$$
\begin{aligned}
U^{2} & V^{2} W^{2} F \\
& \ll U^{2} V^{2} W^{2} \min \left\{V^{-4} W^{-2} M^{2} H, W^{-10} T, W^{-30} H^{5} T, U^{-4} T, U^{-12} K^{2} T\right\} \\
& \leq U^{2} V^{2} W^{2}\left(V^{-4} W^{-2} M^{2} H\right)^{\frac{1}{2}}\left(W^{-30} H^{5} T\right)^{\frac{1}{30}}\left(U^{-4} T\right)^{\frac{9}{20}}\left(U^{-12} K^{2} T\right)^{\frac{1}{60}} \\
& =T^{\frac{1}{2}} M H^{\frac{2}{3}} K^{\frac{1}{30}} \ll x^{1-\varepsilon_{1}} .
\end{aligned}
$$

(e) $F>2 V^{-4} W^{-2} M^{2} H, F \leq 2 W^{-10} H^{5}, 2 U^{-4} K^{2}$. Then

$$
\begin{aligned}
& U^{2} V^{2} W^{2} F \\
& \quad \ll U^{2} V^{2} W^{2} \min \left\{V^{-4} W^{-2} T, V^{-12} W^{-6} M^{2} H T, W^{-10} H^{5}, U^{-4} K^{2}\right\} \\
& \quad \leq U^{2} V^{2} W^{2}\left(V^{-4} W^{-2} T\right)^{\frac{7}{20}}\left(V^{-12} W^{-6} M^{2} H T\right)^{\frac{1}{20}}\left(W^{-10} H^{5}\right)^{\frac{1}{10}}\left(U^{-4} K^{2}\right)^{\frac{1}{2}} \\
& \quad=T^{\frac{2}{5}} M^{\frac{1}{10}} H^{\frac{11}{20}} K \ll x^{1-\varepsilon_{1}} . \\
& \quad \text { (f) } F>2 V^{-4} W^{-2} M^{2} H, F \leq 2 W^{-10} H^{5}, F>2 U^{-4} K^{2} \text {. Then }
\end{aligned}
$$

$$
\begin{aligned}
& U^{2} V^{2} W^{2} F \\
& \ll U^{2} V^{2} W^{2} \min \left\{V^{-4} W^{-2} T, V^{-12} W^{-6} M^{2} H T, W^{-10} H^{5},\right. \\
& \left.\quad U^{-4} T, U^{-12} K^{2} T\right\} \\
& \leq U^{2} V^{2} W^{2}\left(V^{-4} W^{-2} T\right)^{\frac{7}{20}}\left(V^{-12} W^{-6} M^{2} H T\right)^{\frac{1}{20}}\left(W^{-10} H^{5}\right)^{\frac{1}{10}}\left(U^{-4} T\right)^{\frac{1}{2}} \\
& =T^{\frac{9}{10}} M^{\frac{1}{10}} H^{\frac{11}{20}} \ll x^{1-\varepsilon_{1}} .
\end{aligned}
$$


(g) $F>2 V^{-4} W^{-2} M^{2} H, 2 W^{-10} H^{5}, F \leq 2 U^{-4} K^{2}$. Then

$$
\begin{aligned}
& U^{2} V^{2} W^{2} F \\
& \ll U^{2} V^{2} W^{2} \min \left\{V^{-4} W^{-2} T, V^{-12} W^{-6} M^{2} H T, W^{-10} T,\right. \\
& \left.\quad W^{-30} H^{5} T, U^{-4} K^{2}\right\} \\
& \leq U^{2} V^{2} W^{2}\left(V^{-4} W^{-2} T\right)^{\frac{9}{20}}\left(V^{-12} W^{-6} M^{2} H T\right)^{\frac{1}{60}}\left(W^{-30} H^{5} T\right)^{\frac{1}{30}}\left(U^{-4} K^{2}\right)^{\frac{1}{2}} \\
& =T^{\frac{1}{2}} M^{\frac{1}{30}} H^{\frac{11}{60}} K \ll x^{1-\varepsilon_{1}} . \\
& \quad(\mathrm{h}) F>2 V^{-4} W^{-2} M^{2} H, 2 W^{-10} H^{5}, 2 U^{-4} K^{2} . \text { Then } \\
& U^{2} V^{2} W^{2} F \\
& \ll U^{2} V^{2} W^{2} \min \left\{V^{-4} W^{-2}, V^{-12} W^{-6} M^{2} H, W^{-10}, W^{-30} H^{5},\right. \\
& \left.\quad U^{-4}, U^{-12} K^{2}\right\} T \\
& \leq U^{2} V^{2} W^{2}\left(V^{-4} W^{-2}\right)^{\frac{9}{20}}\left(V^{-12} W^{-6} M^{2} H\right)^{\frac{1}{60}}\left(W^{-30} H^{5}\right)^{\frac{1}{30}}\left(U^{-4}\right)^{\frac{1}{2}} T \\
& =T M^{\frac{1}{30}} H^{\frac{11}{60}} \ll x^{1-\varepsilon_{1}} .
\end{aligned}
$$

III. Lastly, we assume condition 3). On applying the mean value estimate and Halász method to $M^{3}(s), H^{4}(s)$ and $K^{2}(s)$, we get

$$
I \ll U^{2} V^{2} W^{2} x^{-1} F \log ^{c} x,
$$

where

$$
\begin{gathered}
F=\min \left\{V^{-6}\left(M^{3}+T\right), V^{-6} M^{3}+V^{-18} M^{3} T, W^{-8}\left(H^{4}+T\right), W^{-8} H^{4}\right. \\
\left.+W^{-24} H^{4} T, U^{-4}\left(K^{2}+T\right), U^{-4} K^{2}+U^{-12} K^{2} T\right\} .
\end{gathered}
$$

Consider the following cases:

(a) $F \leq 2 V^{-6} M^{3}, 2 W^{-8} H^{4}, 2 U^{-4} K^{2}$. Then

$$
\begin{aligned}
U^{2} V^{2} W^{2} F & \ll U^{2} V^{2} W^{2} \min \left\{V^{-6} M^{3}, W^{-8} H^{4}, U^{-4} K^{2}\right\} \\
& \leq U^{2} V^{2} W^{2}\left(V^{-6} M^{3}\right)^{\frac{1}{4}}\left(W^{-8} H^{4}\right)^{\frac{1}{4}}\left(U^{-4} K^{2}\right)^{\frac{1}{2}} \\
& =V^{\frac{1}{2}} M^{\frac{3}{4}} H K \ll x \log ^{-11 B} x .
\end{aligned}
$$

(b) $F \leq 2 V^{-6} M^{3}, 2 W^{-8} H^{4}, F>2 U^{-4} K^{2}$. Then

$$
\begin{aligned}
U^{2} V^{2} W^{2} F & \ll U^{2} V^{2} W^{2} \min \left\{V^{-6} M^{3}, W^{-8} H^{4}, U^{-4} T, U^{-12} K^{2} T\right\} \\
& \leq U^{2} V^{2} W^{2}\left(V^{-6} M^{3}\right)^{\frac{1}{3}}\left(W^{-8} H^{4}\right)^{\frac{1}{4}}\left(U^{-4} T\right)^{\frac{3}{8}}\left(U^{-12} K^{2} T\right)^{\frac{1}{24}} \\
& =T^{\frac{5}{12}} M H K^{\frac{1}{12}} \ll x^{1-\varepsilon_{1}} .
\end{aligned}
$$


(c) $F \leq 2 V^{-6} M^{3}, F>2 W^{-8} H^{4}, F \leq 2 U^{-4} K^{2}$. Then

$$
\begin{aligned}
& U^{2} V^{2} W^{2} F \ll U^{2} V^{2} W^{2} \min \left\{V^{-6} M^{3}, W^{-8} T, W^{-24} H^{4} T, U^{-4} K^{2}\right\} \\
& \quad \leq U^{2} V^{2} W^{2}\left(V^{-6} M^{3}\right)^{\frac{1}{3}}\left(W^{-8} T\right)^{\frac{1}{8}}\left(W^{-24} H^{4} T\right)^{\frac{1}{24}}\left(U^{-4} K^{2}\right)^{\frac{1}{2}} \\
& \quad=T^{\frac{1}{6}} M K H^{\frac{1}{6}} \ll x^{1-\varepsilon_{1}} . \\
& \text { (d) } F \leq 2 V^{-6} M^{3}, F>2 W^{-8} H^{4}, 2 U^{-4} K^{2} . \text { Then } \\
& U^{2} V^{2} W^{2} F \\
& \ll U^{2} V^{2} W^{2} \min \left\{V^{-6} M^{3}, W^{-8} T, W^{-24} H^{4} T, U^{-4} T, U^{-12} K^{2} T\right\} \\
& \leq U^{2} V^{2} W^{2}\left(V^{-6} M^{3}\right)^{\frac{1}{3}}\left(W^{-8} T\right)^{\frac{1}{8}}\left(W^{-24} H^{4} T\right)^{\frac{1}{24}}\left(U^{-4} T\right)^{\frac{1}{2}} \\
& =T^{\frac{2}{3}} M H^{\frac{1}{6}} \ll x^{1-\varepsilon_{1}} .
\end{aligned}
$$

(e) $F>2 V^{-6} M^{3}, F \leq 2 W^{-8} H^{4}, 2 U^{-4} K^{2}$. Then

$$
\begin{aligned}
U^{2} V^{2} W^{2} F & \ll U^{2} V^{2} W^{2} \min \left\{V^{-6} T, V^{-18} M^{3} T, W^{-8} H^{4}, U^{-4} K^{2}\right\} \\
& \leq U^{2} V^{2} W^{2}\left(V^{-6} T\right)^{\frac{5}{24}}\left(V^{-18} M^{3} T\right)^{\frac{1}{24}}\left(W^{-8} H^{4}\right)^{\frac{1}{4}}\left(U^{-4} K^{2}\right)^{\frac{1}{2}} \\
& =T^{\frac{1}{4}} M^{\frac{1}{8}} H K \ll x^{1-\varepsilon_{1}} .
\end{aligned}
$$

(f) $F>2 V^{-6} M^{3}, F \leq 2 W^{-8} H^{4}, F>2 U^{-4} K^{2}$. Then

$$
\begin{aligned}
U^{2} V^{2} W^{2} F & \ll U^{2} V^{2} W^{2} \min \left\{V^{-6} T, V^{-18} M^{3} T, W^{-8} H^{4}, U^{-4} T, U^{-12} K^{2} T\right\} \\
& \leq U^{2} V^{2} W^{2}\left(V^{-6} T\right)^{\frac{5}{24}}\left(V^{-18} M^{3} T\right)^{\frac{1}{24}}\left(W^{-8} H^{4}\right)^{\frac{1}{4}}\left(U^{-4} T\right)^{\frac{1}{2}} \\
& =T^{\frac{3}{4}} M^{\frac{1}{8}} H \ll x^{1-\varepsilon_{1}} .
\end{aligned}
$$

(g) $F>2 V^{-6} M^{3}, 2 W^{-8} H^{4}, F \leq 2 U^{-4} K^{2}$. Then

$$
\begin{aligned}
U^{2} V^{2} & W^{2} F \\
& \ll U^{2} V^{2} W^{2} \min \left\{V^{-6} T, V^{-18} M^{3} T, W^{-8} T, W^{-24} H^{4} T, U^{-4} K^{2}\right\} \\
& \leq U^{2} V^{2} W^{2}\left(V^{-6} T\right)^{\frac{1}{3}}\left(W^{-8} T\right)^{\frac{1}{8}}\left(W^{-24} H^{4} T\right)^{\frac{1}{24}}\left(U^{-4} K^{2}\right)^{\frac{1}{2}} \\
& =T^{\frac{1}{2}} H^{\frac{1}{6}} K \ll x^{1-\varepsilon_{1}} .
\end{aligned}
$$

(h) $F>2 V^{-6} M^{3}, 2 W^{-8} H^{4}, 2 U^{-4} K^{2}$. Then

$$
\begin{aligned}
U^{2} & V^{2} W^{2} F \\
& \ll U^{2} V^{2} W^{2} \min \left\{V^{-6}, V^{-18} M^{3}, W^{-8}, W^{-24} H^{4}, U^{-4}, U^{-12} K^{2}\right\} T \\
& \leq U^{2} V^{2} W^{2}\left(V^{-6}\right)^{\frac{5}{24}}\left(V^{-18} M^{3}\right)^{\frac{1}{24}}\left(W^{-8}\right)^{\frac{1}{4}}\left(U^{-4}\right)^{\frac{1}{2}} T \\
& =T M^{\frac{1}{8}} \ll x^{1-\varepsilon_{1}},
\end{aligned}
$$

since $M \ll X^{\frac{2}{5}}$ (the latter follows from $M H \ll X^{\frac{25}{44}}$ and $\left.X^{\frac{19}{100}} \ll H\right)$.

Combining the above, we obtain (8). Hence, Lemma 5 follows. 
Lemma 6. Under the assumption of Lemma 5, $M$ and $H$ lie in one of the following regions:

(i) $\quad X^{\frac{19}{70}} \ll M \ll X^{\frac{3}{10}}, \quad M^{-\frac{6}{5}} X^{\frac{57}{100}} \ll H \ll M^{-\frac{1}{8}} X^{\frac{23}{80}}$;

(ii) $\quad X^{\frac{3}{10}} \ll M \ll X^{\frac{19}{60}}, \quad M^{-\frac{12}{11}} X^{\frac{57}{110}} \ll H \ll M^{-\frac{1}{8}} X^{\frac{23}{80}}$;

(iii) $\quad X^{\frac{19}{60}} \ll M \ll X^{\frac{247}{770}}, \quad X^{\frac{19}{110}} \ll H \ll M^{-\frac{1}{8}} X^{\frac{23}{80}}$;

(iv) $\quad X^{\frac{247}{770}} \ll M \ll X^{\frac{359}{1100}}, \quad X^{\frac{19}{110}} \ll H \ll M^{-1} X^{\frac{25}{44}}$;

(v) $\quad X^{\frac{359}{1100}} \ll M \ll X^{\frac{481}{1450}}, \quad X^{\frac{19}{110}} \ll H \ll M^{-6} X^{\frac{11}{5}}$;

(vi) $\quad X^{\frac{481}{1450}} \ll M \ll X^{\frac{443}{1305}}, \quad X^{\frac{19}{110}} \ll H \ll M^{-1} X^{\frac{157}{290}}$;

(vii) $\quad X^{\frac{443}{1305}} \ll M \ll X^{\frac{19}{55}}, \quad X^{\frac{19}{110}} \ll H \ll M^{-\frac{2}{11}} X^{\frac{29}{110}}$;

(viii) $\quad X^{\frac{19}{55}} \ll M \ll X^{\frac{2143}{5620}}, \quad M^{-\frac{58}{49}} X^{\frac{57}{98}} \ll H \ll M^{-\frac{2}{11}} X^{\frac{29}{110}}$;

(ix) $\quad X^{\frac{2143}{5620}} \ll M \ll X^{\frac{3963}{9860}}, \quad M^{-\frac{58}{49}} X^{\frac{57}{98}} \ll H \ll M^{-\frac{29}{19}} X^{\frac{59}{76}}$;

(x) $\quad X^{\frac{3963}{9860}} \ll M \ll X^{\frac{4331}{9860}}, \quad X^{\frac{9}{85}} \ll H \ll M^{-\frac{29}{19}} X^{\frac{59}{76}}$.

Then (7) holds for $T_{0} \leq T \leq X$.

Pro of. In the regions:

$$
\begin{array}{ll}
X^{\frac{3}{10}} \ll M \ll X^{\frac{19}{60}}, & M^{-\frac{12}{11}} X^{\frac{57}{110}} \ll H \ll M^{\frac{1}{29}} X^{\frac{31}{145}} ; \\
X^{\frac{19}{60}} \ll M \ll X^{\frac{19}{55}}, & X^{\frac{19}{110}} \ll H \ll M^{-1} X^{\frac{157}{290}},
\end{array}
$$

we apply Lemma 5 with condition 1 ).

In the regions:

$$
\begin{array}{ll}
X^{\frac{443}{1305}} \ll M \ll X^{\frac{19}{55}}, & M^{-1} X^{\frac{157}{290}} \ll H \ll M^{-\frac{2}{11}} X^{\frac{29}{110}} \\
X^{\frac{19}{55}} \ll M \ll X^{\frac{2143}{5620}}, & M^{-\frac{58}{49}} X^{\frac{57}{98}} \ll H \ll M^{-\frac{2}{11}} X^{\frac{29}{110}} \\
X^{\frac{2143}{5620}} \ll M \ll X^{\frac{3963}{9860}}, & M^{-\frac{58}{49}} X^{\frac{57}{98}} \ll H \ll M^{-\frac{29}{19}} X^{\frac{59}{76}} \\
X^{\frac{3963}{9860}} \ll M \ll X^{\frac{4331}{9860}}, & X^{\frac{9}{85}} \ll H \ll M^{-\frac{29}{19}} X^{\frac{59}{76}},
\end{array}
$$

we apply Lemma 5 with condition 2).

In the regions:

$$
\begin{array}{ll}
X^{\frac{19}{70}} \ll M \ll X^{\frac{3}{10}}, & M^{-\frac{6}{5}} X^{\frac{57}{100}} \ll H \ll M^{-\frac{1}{8}} X^{\frac{23}{80}} ; \\
X^{\frac{3}{10}} \ll M \ll X^{\frac{19}{60}}, & M^{\frac{1}{29}} X^{\frac{31}{145}} \ll H \ll M^{-\frac{1}{8}} X^{\frac{23}{80}} ; \\
X^{\frac{19}{60}} \ll M \ll X^{\frac{247}{770}}, & M^{-1} X^{\frac{157}{290}} \ll H \ll M^{-\frac{1}{8}} X^{\frac{23}{80}} ; \\
X^{\frac{247}{770}} \ll M \ll X^{\frac{359}{1100}}, & M^{-1} X^{\frac{157}{290}} \ll H \ll M^{-1} X^{\frac{25}{44}} ; \\
X^{\frac{359}{1100}} \ll M \ll X^{\frac{481}{1450}}, & M^{-1} X^{\frac{157}{290}} \ll H \ll M^{-6} X^{\frac{11}{5}},
\end{array}
$$

we apply Lemma 5 with condition 3 ).

Putting together the above regions, we get Lemma 6 . 
Lemma 7. Under the assumption of Lemma 5, suppose that $M$ and $H$ also satisfy one of the following three conditions:

1) $M H \ll X^{\frac{7}{10}}, X^{\frac{19}{110}} \ll H, M^{12} H \ll X^{\frac{63}{10}}, X^{\frac{133}{290}} \ll M, H^{19} / M \ll$ $X^{\frac{19}{5}}, X^{\frac{38}{5}} \ll M^{12} H^{11}$

2) $M^{2} H \ll X^{\frac{52}{45}}, M^{58} H^{9} \ll X^{\frac{59}{2}}, X^{\frac{19}{45}} \ll M, M H^{5} \ll X^{\frac{29}{20}}, X^{\frac{57}{4}} \ll$ $M^{29} H^{10}$

3) $M H \ll X^{\frac{51}{70}}, X^{\frac{19}{100}} \ll H, M^{6} H \ll X^{\frac{63}{20}}, X^{\frac{19}{44}} \ll M, H^{7} / M \ll X^{\frac{13}{10}}$, $X^{\frac{19}{5}} \ll M^{6} H^{5}$.

Then (7) holds for $T_{0} \leq T \leq X$.

Proof. We only show that (8) holds for $T=1 / \eta=2 X^{\frac{19}{20}-\varepsilon}$.

I. First, we assume condition 1$)$. We apply the mean value estimate and Halász method to $M^{2}(s), H^{5}(s)$ and $K^{3}(s)$ to get

$$
I \ll U^{2} V^{2} W^{2} x^{-1} F \log ^{c} x,
$$

where

$$
\begin{gathered}
F=\min \left\{V^{-4}\left(M^{2}+T\right), V^{-4} M^{2}+V^{-12} M^{2} T, W^{-10}\left(H^{5}+T\right), W^{-10} H^{5}\right. \\
\left.+W^{-30} H^{5} T, U^{-6}\left(K^{3}+T\right), U^{-6} K^{3}+U^{-18} K^{3} T\right\} .
\end{gathered}
$$

We consider several cases:

(a) $F \leq 2 V^{-4} M^{2}, 2 W^{-10} H^{5}, 2 U^{-6} K^{3}$. Then

$$
\begin{aligned}
U^{2} V^{2} W^{2} F & \ll U^{2} V^{2} W^{2} \min \left\{V^{-4} M^{2}, W^{-10} H^{5}, U^{-6} K^{3}\right\} \\
& \leq U^{2} V^{2} W^{2}\left(V^{-4} M^{2}\right)^{\frac{1}{2}}\left(W^{-10} H^{5}\right)^{\frac{1}{6}}\left(U^{-6} K^{3}\right)^{\frac{1}{3}} \\
& =W^{\frac{1}{3}} H^{\frac{5}{6}} M K \ll x \log ^{-11 B} x .
\end{aligned}
$$

(b) $F \leq 2 V^{-4} M^{2}, 2 W^{-10} H^{5}, F>2 U^{-6} K^{3}$. Then

$$
\begin{aligned}
U^{2} V^{2} W^{2} F & \ll U^{2} V^{2} W^{2} \min \left\{V^{-4} M^{2}, W^{-10} H^{5}, U^{-6} T, U^{-18} K^{3} T\right\} \\
& \leq U^{2} V^{2} W^{2}\left(V^{-4} M^{2}\right)^{\frac{1}{2}}\left(W^{-10} H^{5}\right)^{\frac{1}{5}}\left(U^{-6} T\right)^{\frac{17}{60}}\left(U^{-18} K^{3} T\right)^{\frac{1}{60}} \\
& =T^{\frac{3}{10}} M H K^{\frac{1}{20}} \ll x^{1-\varepsilon_{1}} . \\
(\text { c) } F \leq 2 & V^{-4} M^{2}, F>2 W^{-10} H^{5}, F \leq 2 U^{-6} K^{3} . \text { Then } \\
U^{2} V^{2} W^{2} F & \ll U^{2} V^{2} W^{2} \min \left\{V^{-4} M^{2}, W^{-10} T, W^{-30} H^{5} T, U^{-6} K^{3}\right\} \\
& \leq U^{2} V^{2} W^{2}\left(V^{-4} M^{2}\right)^{\frac{1}{2}}\left(W^{-10} T\right)^{\frac{3}{20}}\left(W^{-30} H^{5} T\right)^{\frac{1}{60}}\left(U^{-6} K^{3}\right)^{\frac{1}{3}} \\
& =T^{\frac{1}{6}} M K H^{\frac{1}{12}} \ll x^{1-\varepsilon_{1}} .
\end{aligned}
$$


(d) $F \leq 2 V^{-4} M^{2}, F>2 W^{-10} H^{5}, 2 U^{-6} K^{3}$. Then

$$
\begin{aligned}
U^{2} V^{2} & W^{2} F \\
& \ll U^{2} V^{2} W^{2} \min \left\{V^{-4} M^{2}, W^{-10} T, W^{-30} H^{5} T, U^{-6} T, U^{-18} K^{3} T\right\} \\
& \leq U^{2} V^{2} W^{2}\left(V^{-4} M^{2}\right)^{\frac{1}{2}}\left(W^{-10} T\right)^{\frac{3}{20}}\left(W^{-30} H^{5} T\right)^{\frac{1}{60}}\left(U^{-6} T\right)^{\frac{1}{3}} \\
& =T^{\frac{1}{2}} M H^{\frac{1}{12}} \ll x^{1-\varepsilon_{1}} .
\end{aligned}
$$

(e) $F>2 V^{-4} M^{2}, F \leq 2 W^{-10} H^{5}, 2 U^{-6} K^{3}$. Then

$$
\begin{aligned}
U^{2} V^{2} W^{2} F & \ll U^{2} V^{2} W^{2} \min \left\{V^{-4} T, V^{-12} M^{2} T, W^{-10} H^{5}, U^{-6} K^{3}\right\} \\
& \leq U^{2} V^{2} W^{2}\left(V^{-4} T\right)^{\frac{9}{20}}\left(V^{-12} M^{2} T\right)^{\frac{1}{60}}\left(W^{-10} H^{5}\right)^{\frac{1}{5}}\left(U^{-6} K^{3}\right)^{\frac{1}{3}} \\
& =T^{\frac{7}{15}} M^{\frac{1}{30}} H K \ll x^{1-\varepsilon_{1}} .
\end{aligned}
$$

(f) $F>2 V^{-4} M^{2}, F \leq 2 W^{-10} H^{5}, F>2 U^{-6} K^{3}$. Then

$$
\begin{aligned}
U^{2} V^{2} & W^{2} F \\
& \ll U^{2} V^{2} W^{2} \min \left\{V^{-4} T, V^{-12} M^{2} T, W^{-10} H^{5}, U^{-6} T, U^{-18} K^{3} T\right\} \\
& \leq U^{2} V^{2} W^{2}\left(V^{-4} T\right)^{\frac{1}{2}}\left(W^{-10} H^{5}\right)^{\frac{1}{5}}\left(U^{-6} T\right)^{\frac{17}{60}}\left(U^{-18} K^{3} T\right)^{\frac{1}{60}} \\
& =T^{\frac{4}{5}} H K^{\frac{1}{20}} \ll x^{1-\varepsilon_{1}} . \\
\text { (g) } F & >2 V^{-4} M^{2}, 2 W^{-10} H^{5}, F \leq 2 U^{-6} K^{3} \text {. Then } \\
U^{2} V^{2} & W^{2} F \\
& \ll U^{2} V^{2} W^{2} \min \left\{V^{-4} T, V^{-12} M^{2} T, W^{-10} T, W^{-30} H^{5} T, U^{-6} K^{3}\right\} \\
& \leq U^{2} V^{2} W^{2}\left(V^{-4} T\right)^{\frac{1}{2}}\left(W^{-10} T\right)^{\frac{3}{20}}\left(W^{-30} H^{5} T\right)^{\frac{1}{60}}\left(U^{-6} K^{3}\right)^{\frac{1}{3}} \\
& =T^{\frac{2}{3}} H^{\frac{1}{12}} K \ll x^{1-\varepsilon_{1}} .
\end{aligned}
$$

(h) $F>2 V^{-4} M^{2}, 2 W^{-10} H^{5}, 2 U^{-6} K^{3}$. Then

$$
\begin{aligned}
& U^{2} V^{2} W^{2} F \\
& \quad \ll U^{2} V^{2} W^{2} \min \left\{V^{-4}, V^{-12} M^{2}, W^{-10}, W^{-30} H^{5}, U^{-6}, U^{-18} K^{3}\right\} T \\
& \quad \leq U^{2} V^{2} W^{2}\left(V^{-4}\right)^{\frac{1}{2}}\left(W^{-10}\right)^{\frac{1}{5}}\left(U^{-6}\right)^{\frac{17}{60}}\left(U^{-18} K^{3}\right)^{\frac{1}{60}} T \\
& \quad=T K^{\frac{1}{20}} \ll x^{1-\varepsilon_{1}},
\end{aligned}
$$

since $K \ll X$.

II. Next, assume condition 2). We apply the mean value estimate and Halász method to $M^{2}(s), H^{5}(s)$ and $K^{2}(s) H(s)$ to get

$$
I \ll U^{2} V^{2} W^{2} x^{-1} F \log ^{c} x,
$$


where

$$
\begin{aligned}
F=\min \{ & V^{-4}\left(M^{2}+T\right), V^{-4} M^{2}+V^{-12} M^{2} T, W^{-10}\left(H^{5}+T\right), W^{-10} H^{5} \\
& +W^{-30} H^{5} T, U^{-4} W^{-2}\left(K^{2} H+T\right), U^{-4} W^{-2} K^{2} H \\
& \left.+U^{-12} W^{-6} K^{2} H T\right\} .
\end{aligned}
$$

Consider the following cases:

(a) $F \leq 2 V^{-4} M^{2}, 2 W^{-10} H^{5}, 2 U^{-4} W^{-2} K^{2} H$. Then

$$
\begin{aligned}
U^{2} V^{2} W^{2} F & \ll U^{2} V^{2} W^{2} \min \left\{V^{-4} M^{2}, W^{-10} H^{5}, U^{-4} W^{-2} K^{2} H\right\} \\
& \leq U^{2} V^{2} W^{2}\left(V^{-4} M^{2}\right)^{\frac{1}{2}}\left(U^{-4} W^{-2} K^{2} H\right)^{\frac{1}{2}} \\
& =W H^{\frac{1}{2}} M K \ll x \log ^{-11 B} x .
\end{aligned}
$$

(b) $F \leq 2 V^{-4} M^{2}, 2 W^{-10} H^{5}, F>2 U^{-4} W^{-2} K^{2} H$. Then

$$
U^{2} V^{2} W^{2} F
$$

$\ll U^{2} V^{2} W^{2} \min \left\{V^{-4} M^{2}, W^{-10} H^{5}, U^{-4} W^{-2} T, U^{-12} W^{-6} K^{2} H T\right\}$

$\leq U^{2} V^{2} W^{2}\left(V^{-4} M^{2}\right)^{\frac{1}{2}}\left(W^{-10} H^{5}\right)^{\frac{1}{10}}\left(U^{-4} W^{-2} T\right)^{\frac{7}{20}}\left(U^{-12} W^{-6} K^{2} H T\right)^{\frac{1}{20}}$

$=T^{\frac{2}{5}} M H^{\frac{11}{20}} K^{\frac{1}{10}} \ll x^{1-\varepsilon_{1}}$.

(c) $F \leq 2 V^{-4} M^{2}, F>2 W^{-10} H^{5}, F \leq 2 U^{-4} W^{-2} K^{2} H$. Then

$$
\begin{aligned}
U^{2} V^{2} W^{2} F & \ll U^{2} V^{2} W^{2} \min \left\{V^{-4} M^{2}, W^{-10} T, W^{-30} H^{5} T, U^{-4} W^{-2} K^{2} H\right\} \\
& \leq U^{2} V^{2} W^{2}\left(V^{-4} M^{2}\right)^{\frac{1}{2}}\left(U^{-4} W^{-2} K^{2} H\right)^{\frac{1}{2}} \\
& =W H^{\frac{1}{2}} M K \ll x \log ^{-11 B} x .
\end{aligned}
$$

(d) $F \leq 2 V^{-4} M^{2}, F>2 W^{-10} H^{5}, 2 U^{-4} W^{-2} K^{2} H$. Then $U^{2} V^{2} W^{2} F$ $\ll U^{2} V^{2} W^{2} \min \left\{V^{-4} M^{2}, W^{-10} T, W^{-30} H^{5} T, U^{-4} W^{-2} T\right.$,

$$
\left.U^{-12} W^{-6} K^{2} H T\right\}
$$$$
\leq U^{2} V^{2} W^{2}\left(V^{-4} M^{2}\right)^{\frac{1}{2}}\left(W^{-30} H^{5} T\right)^{\frac{1}{30}}\left(U^{-4} W^{-2} T\right)^{\frac{9}{20}}\left(U^{-12} W^{-6} K^{2} H T\right)^{\frac{1}{60}}
$$$$
=T^{\frac{1}{2}} M H^{\frac{11}{60}} K^{\frac{1}{30}} \ll x^{1-\varepsilon_{1}} \text {. }
$$

(e) $F>2 V^{-4} M^{2}, F \leq 2 W^{-10} H^{5}, 2 U^{-4} W^{-2} K^{2} H$. Then

$$
\begin{aligned}
& U^{2} V^{2} W^{2} F \\
& \quad \ll U^{2} V^{2} W^{2} \min \left\{V^{-4} T, V^{-12} M^{2} T, W^{-10} H^{5}, U^{-4} W^{-2} K^{2} H\right\} \\
& \quad \leq U^{2} V^{2} W^{2}\left(V^{-4} T\right)^{\frac{7}{20}}\left(V^{-12} M^{2} T\right)^{\frac{1}{20}}\left(W^{-10} H^{5}\right)^{\frac{1}{10}}\left(U^{-4} W^{-2} K^{2} H\right)^{\frac{1}{2}} \\
& =T^{\frac{2}{5}} M^{\frac{1}{10}} H K \ll x^{1-\varepsilon_{1}} .
\end{aligned}
$$


(f) $F>2 V^{-4} M^{2}, F \leq 2 W^{-10} H^{5}, F>2 U^{-4} W^{-2} K^{2} H$. Then

$$
\begin{aligned}
& U^{2} V^{2} W^{2} F \\
& \ll U^{2} V^{2} W^{2} \min \left\{V^{-4} T, V^{-12} M^{2} T, W^{-10} H^{5}, U^{-4} W^{-2} T,\right. \\
& \left.\quad U^{-12} W^{-6} K^{2} H T\right\} \\
& \leq U^{2} V^{2} W^{2}\left(V^{-4} T\right)^{\frac{7}{20}}\left(V^{-12} M^{2} T\right)^{\frac{1}{20}}\left(W^{-10} H^{5}\right)^{\frac{1}{10}}\left(U^{-4} W^{-2} T\right)^{\frac{1}{2}} \\
& =T^{\frac{9}{10}} M^{\frac{1}{10}} H^{\frac{1}{2}} \ll x^{1-\varepsilon_{1}} .
\end{aligned}
$$

(g) $F>2 V^{-4} M^{2}, 2 W^{-10} H^{5}, F \leq 2 U^{-4} W^{-2} K^{2} H$. Then

$$
\begin{aligned}
& U^{2} V^{2} W^{2} F \\
& \ll U^{2} V^{2} W^{2} \min \left\{V^{-4} T, V^{-12} M^{2} T, W^{-10} T, W^{-30} H^{5} T, U^{-4} W^{-2} K^{2} H\right\} \\
& \leq U^{2} V^{2} W^{2}\left(V^{-4} T\right)^{\frac{9}{20}}\left(V^{-12} M^{2} T\right)^{\frac{1}{60}}\left(W^{-30} H^{5} T\right)^{\frac{1}{30}}\left(U^{-4} W^{-2} K^{2} H\right)^{\frac{1}{2}} \\
& =T^{\frac{1}{2}} M^{\frac{1}{30}} H^{\frac{2}{3}} K \ll x^{1-\varepsilon_{1}} .
\end{aligned}
$$

(h) $F>2 V^{-4} M^{2}, 2 W^{-10} H^{5}, 2 U^{-4} W^{-2} K^{2} H$. Then

$$
\begin{aligned}
U^{2} V^{2} W^{2} F & \ll U^{2} V^{2} W^{2} \min \left\{V^{-4}, V^{-12} M^{2}, W^{-10}, W^{-30} H^{5}, U^{-4} W^{-2},\right. \\
& \left.U^{-12} W^{-6} K^{2} H\right\} T \\
\leq & U^{2} V^{2} W^{2}\left(V^{-4}\right)^{\frac{9}{20}}\left(V^{-12} M^{2}\right)^{\frac{1}{60}}\left(W^{-30} H^{5}\right)^{\frac{1}{30}}\left(U^{-4} W^{-2}\right)^{\frac{1}{2}} T \\
& =T M^{\frac{1}{30}} H^{\frac{1}{6}} \ll x^{1-\varepsilon_{1}} .
\end{aligned}
$$

III. Lastly, we assume condition 3). We apply the mean value estimate and Halász method to $M^{2}(s), H^{4}(s)$ and $K^{3}(s)$ to get

$$
I \ll U^{2} V^{2} W^{2} x^{-1} F \log ^{c} x,
$$

where

$$
\begin{gathered}
F=\min \left\{V^{-4}\left(M^{2}+T\right), V^{-4} M^{2}+V^{-12} M^{2} T, W^{-8}\left(H^{4}+T\right), W^{-8} H^{4}\right. \\
\left.+W^{-24} H^{4} T, U^{-6}\left(K^{3}+T\right), U^{-6} K^{3}+U^{-18} K^{3} T\right\} .
\end{gathered}
$$

Consider the following cases:

(a) $F \leq 2 V^{-4} M^{2}, 2 W^{-8} H^{4}, 2 U^{-6} K^{3}$. Then

$$
\begin{aligned}
U^{2} V^{2} W^{2} F & \ll U^{2} V^{2} W^{2} \min \left\{V^{-4} M^{2}, W^{-8} H^{4}, U^{-6} K^{3}\right\} \\
& \leq U^{2} V^{2} W^{2}\left(V^{-4} M^{2}\right)^{\frac{1}{2}}\left(W^{-8} H^{4}\right)^{\frac{1}{6}}\left(U^{-6} K^{3}\right)^{\frac{1}{3}} \\
& =W^{\frac{2}{3}} H^{\frac{2}{3}} M K \ll x \log ^{-11 B} x .
\end{aligned}
$$


(b) $F \leq 2 V^{-4} M^{2}, 2 W^{-8} H^{4}, F>2 U^{-6} K^{3}$. Then

$$
\begin{aligned}
U^{2} V^{2} W^{2} F & \ll U^{2} V^{2} W^{2} \min \left\{V^{-4} M^{2}, W^{-8} H^{4}, U^{-6} T, U^{-18} K^{3} T\right\} \\
& \leq U^{2} V^{2} W^{2}\left(V^{-4} M^{2}\right)^{\frac{1}{2}}\left(W^{-8} H^{4}\right)^{\frac{1}{4}}\left(U^{-6} T\right)^{\frac{5}{24}}\left(U^{-18} K^{3} T\right)^{\frac{1}{24}} \\
& =T^{\frac{1}{4}} M H K^{\frac{1}{8}} \ll x^{1-\varepsilon_{1}} .
\end{aligned}
$$

(c) $F \leq 2 V^{-4} M^{2}, F>2 W^{-8} H^{4}, F \leq 2 U^{-6} K^{3}$. Then

$$
\begin{aligned}
U^{2} V^{2} W^{2} F & \ll U^{2} V^{2} W^{2} \min \left\{V^{-4} M^{2}, W^{-8} T, W^{-24} H^{4} T, U^{-6} K^{3}\right\} \\
& \leq U^{2} V^{2} W^{2}\left(V^{-4} M^{2}\right)^{\frac{1}{2}}\left(W^{-8} T\right)^{\frac{1}{8}}\left(W^{-24} H^{4} T\right)^{\frac{1}{2^{24}}}\left(U^{-6} K^{3}\right)^{\frac{1}{3}} \\
& =T^{\frac{1}{6}} M K H^{\frac{1}{6}} \ll x^{1-\varepsilon_{1}} .
\end{aligned}
$$

(d) $F \leq 2 V^{-4} M^{2}, F>2 W^{-8} H^{4}, 2 U^{-6} K^{3}$. Then

$$
\begin{aligned}
U^{2} V^{2} & W^{2} F \\
& \ll U^{2} V^{2} W^{2} \min \left\{V^{-4} M^{2}, W^{-8} T, W^{-24} H^{4} T, U^{-6} T, U^{-18} K^{3} T\right\} \\
& \leq U^{2} V^{2} W^{2}\left(V^{-4} M^{2}\right)^{\frac{1}{2}}\left(W^{-8} T\right)^{\frac{1}{8}}\left(W^{-24} H^{4} T\right)^{\frac{1}{24}}\left(U^{-6} T\right)^{\frac{1}{3}} \\
& =T^{\frac{1}{2}} M H^{\frac{1}{6}} \ll x^{1-\varepsilon_{1}} .
\end{aligned}
$$

(e) $F>2 V^{-4} M^{2}, F \leq 2 W^{-8} H^{4}, 2 U^{-6} K^{3}$. Then

$$
\begin{aligned}
U^{2} V^{2} W^{2} F & \ll U^{2} V^{2} W^{2} \min \left\{V^{-4} T, V^{-12} M^{2} T, W^{-8} H^{4}, U^{-6} K^{3}\right\} \\
& \leq U^{2} V^{2} W^{2}\left(V^{-4} T\right)^{\frac{3}{8}}\left(V^{-12} M^{2} T\right)^{\frac{1}{24}}\left(W^{-8} H^{4}\right)^{\frac{1}{4}}\left(U^{-6} K^{3}\right)^{\frac{1}{3}} \\
& =T^{\frac{5}{12}} M^{\frac{1}{12}} H K \ll x^{1-\varepsilon_{1}} .
\end{aligned}
$$

(f) $F>2 V^{-4} M^{2}, F \leq 2 W^{-8} H^{4}, F>2 U^{-6} K^{3}$. Then

$$
\begin{aligned}
U^{2} V^{2} W^{2} F & \ll U^{2} V^{2} W^{2} \min \left\{V^{-4} T, V^{-12} M^{2} T, W^{-8} H^{4}, U^{-6} T, U^{-18} K^{3} T\right\} \\
& \leq U^{2} V^{2} W^{2}\left(V^{-4} T\right)^{\frac{1}{2}}\left(W^{-8} H^{4}\right)^{\frac{1}{4}}\left(U^{-6} T\right)^{\frac{5}{24}}\left(U^{-18} K^{3} T\right)^{\frac{1}{24}} \\
& =T^{\frac{3}{4}} H K^{\frac{1}{8}} \ll x^{1-\varepsilon_{1}} .
\end{aligned}
$$

(g) $F>2 V^{-4} M^{2}, 2 W^{-8} H^{4}, F \leq 2 U^{-6} K^{3}$. Then

$$
\begin{aligned}
U^{2} V^{2} & W^{2} F \\
& \ll U^{2} V^{2} W^{2} \min \left\{V^{-4} T, V^{-12} M^{2} T, W^{-8} T, W^{-24} H^{4} T, U^{-6} K^{3}\right\} \\
& \leq U^{2} V^{2} W^{2}\left(V^{-4} T\right)^{\frac{1}{2}}\left(W^{-8} T\right)^{\frac{1}{8}}\left(W^{-24} H^{4} T\right)^{\frac{1}{24}}\left(U^{-6} K^{3}\right)^{\frac{1}{3}} \\
& =T^{\frac{2}{3}} H^{\frac{1}{6}} K \ll x^{1-\varepsilon_{1}} .
\end{aligned}
$$


(h) $F>2 V^{-4} M^{2}, 2 W^{-8} H^{4}, 2 U^{-6} K^{3}$. Then

$$
\begin{aligned}
& U^{2} V^{2} W^{2} F \\
& \quad \ll U^{2} V^{2} W^{2} \min \left\{V^{-4}, V^{-12} M^{2}, W^{-8}, W^{-24} H^{4}, U^{-6}, U^{-18} K^{3}\right\} T \\
& \quad \leq U^{2} V^{2} W^{2}\left(V^{-4}\right)^{\frac{1}{2}}\left(W^{-8}\right)^{\frac{1}{4}}\left(U^{-6}\right)^{\frac{5}{24}}\left(U^{-18} K^{3}\right)^{\frac{1}{24}} T \\
& \quad=T K^{\frac{1}{8}} \ll x^{1-\varepsilon_{1}},
\end{aligned}
$$

since $X^{\frac{3}{5}} \ll M H$ (the latter follows from $X^{\frac{19}{44}} \ll M$ and $\left.X^{\frac{19}{100}} \ll H\right)$.

Combining the above, we obtain (8). Hence, Lemma 7 follows.

LEMMA 8. Under the assumption of Lemma 5, suppose that $M$ and $H$ lie in one of the following regions:

(i) $\quad X^{\frac{19}{45}} \ll M \ll X^{\frac{19}{44}}, \quad M^{-\frac{29}{10}} X^{\frac{57}{40}} \ll H \ll M^{-\frac{1}{5}} X^{\frac{29}{100}}$;

(ii) $\quad X^{\frac{19}{44}} \ll M \ll X^{\frac{897}{1972}}, \quad M^{-\frac{29}{10}} X^{\frac{57}{40}} \ll H \ll M^{-\frac{1}{5}} X^{\frac{29}{100}}$;

(iii) $\quad X^{\frac{19}{44}} \ll M \ll X^{\frac{897}{1972}}, \quad M^{-\frac{6}{5}} X^{\frac{19}{25}} \ll H \ll M^{\frac{1}{7}} X^{\frac{13}{70}}$;

(iv) $\quad X^{\frac{897}{1972}} \ll M \ll X^{\frac{133}{290}}, \quad X^{\frac{9}{85}} \ll H \ll M^{-\frac{1}{5}} X^{\frac{29}{100}}$;

(v) $\quad X^{\frac{897}{1972}} \ll M \ll X^{\frac{133}{290}}, \quad M^{-\frac{6}{5}} X^{\frac{19}{25}} \ll H \ll M^{\frac{1}{7}} X^{\frac{13}{70}}$;

(vi) $\quad X^{\frac{133}{290}} \ll M \ll X^{\frac{19}{40}}, \quad X^{\frac{9}{85}} \ll H \ll M^{\frac{1}{7}} X^{\frac{13}{70}}$;

(vii) $\quad X^{\frac{19}{40}} \ll M \ll X^{\frac{53}{110}}, \quad X^{\frac{9}{85}} \ll H \ll M^{-1} X^{\frac{51}{70}}$;

(viii) $\quad X^{\frac{53}{110}} \ll M \ll X^{\frac{339}{700}}, \quad X^{\frac{9}{85}} \ll H \ll M^{-\frac{58}{9}} X^{\frac{59}{18}}$;

(ix) $\quad X^{\frac{53}{110}} \ll M \ll X^{\frac{339}{700}}, \quad X^{\frac{19}{110}} \ll H \ll M^{-1} X^{\frac{51}{70}}$;

(x) $\quad X^{\frac{339}{700}} \ll M \ll X^{\frac{49}{100}}, \quad X^{\frac{9}{85}} \ll H \ll M^{-\frac{58}{9}} X^{\frac{59}{18}} ;$

(xi) $\quad X^{\frac{339}{700}} \ll M \ll X^{\frac{49}{100}}, \quad X^{\frac{19}{110}} \ll H \ll M^{-6} X^{\frac{63}{20}} ;$

(xii) $\quad X^{\frac{49}{100}} \ll M \ll X^{\frac{1}{2}}, \quad X^{\frac{19}{110}} \ll H \ll M^{-1} X^{\frac{7}{10}}$.

Then (7) holds for $T_{0} \leq T \leq X$.

Pr o of. In the regions:

$$
\begin{array}{ll}
X^{\frac{133}{290}} \ll M \ll X^{\frac{19}{40}}, & M^{-\frac{12}{11}} X^{\frac{38}{55}} \ll H \ll M^{\frac{1}{19}} X^{\frac{1}{5}} ; \\
X^{\frac{19}{40}} \ll M \ll X^{\frac{1}{2}}, & X^{\frac{19}{110}} \ll H \ll M^{-1} X^{\frac{7}{10}},
\end{array}
$$

we apply Lemma 7 with condition 1 ).

In the regions:

$$
\begin{array}{ll}
X^{\frac{19}{45}} \ll M \ll X^{\frac{897}{1972}}, & M^{-\frac{29}{10}} X^{\frac{57}{40}} \ll H \ll M^{-\frac{1}{5}} X^{\frac{29}{100}} ; \\
X^{\frac{897}{1972}} \ll M \ll X^{\frac{133}{290}}, & X^{\frac{9}{85}} \ll H \ll M^{-\frac{1}{5}} X^{\frac{29}{100}} ; \\
X^{\frac{133}{290}} \ll M \ll X^{\frac{19}{40}}, & X^{\frac{9}{85}} \ll H \ll M^{-\frac{12}{11}} X^{\frac{38}{55}} ;
\end{array}
$$




$$
\begin{array}{ll}
X^{\frac{19}{40}} \ll M \ll X^{\frac{53}{110}}, & X^{\frac{9}{85}} \ll H \ll X^{\frac{19}{110}} ; \\
X^{\frac{53}{110}} \ll M \ll X^{\frac{49}{100}}, & X^{\frac{9}{85}} \ll H \ll M^{-\frac{58}{9}} X^{\frac{59}{18}},
\end{array}
$$

we apply Lemma 7 with condition 2).

In the regions:

$$
\begin{array}{ll}
X^{\frac{19}{44}} \ll M \ll X^{\frac{133}{290}}, & M^{-\frac{6}{5}} X^{\frac{19}{25}} \ll H \ll M^{\frac{1}{7}} X^{\frac{13}{70}} \\
X^{\frac{133}{290}} \ll M \ll X^{\frac{19}{40}}, & M^{\frac{1}{19}} X^{\frac{1}{5}} \ll H \ll M^{\frac{1}{7}} X^{\frac{13}{70}} ; \\
X^{\frac{19}{40}} \ll M \ll X^{\frac{339}{700}}, & M^{-1} X^{\frac{7}{10}} \ll H \ll M^{-1} X^{\frac{51}{70}} ; \\
X^{\frac{339}{700}} \ll M \ll X^{\frac{49}{100}}, & M^{-1} X^{\frac{7}{10}} \ll H \ll M^{-6} X^{\frac{63}{20}},
\end{array}
$$

we apply Lemma 7 with condition 3 ).

Putting together the above regions, we get Lemma 8.

Lemma 9. Under the assumption of Lemma 5, suppose that $M$ and $H$ lie in one of the following regions:

(i) $\quad X^{\frac{1843}{5280}} \ll M \ll X^{\frac{1188}{2975}}, \quad M^{-\frac{70}{59}} X^{\frac{171}{295}} \ll H \ll M^{-\frac{58}{49}} X^{\frac{57}{98}}$;

(ii) $\quad X^{\frac{1188}{2975}} \ll M \ll X^{\frac{3963}{9860}}, \quad X^{\frac{9}{85}} \ll H \ll M^{-\frac{58}{49}} X^{\frac{57}{98}}$;

(iii) $\quad X^{\frac{1063}{2640}} \ll M \ll X^{\frac{4331}{9860}}, \quad M^{-\frac{29}{19}} X^{\frac{59}{76}} \ll H \ll M^{-\frac{35}{23}} X^{\frac{179}{230}}$.

Then (7) holds for $T_{0} \leq T \leq X$.

Proof. First we show that (8) holds for $T=1 / \eta=2 X^{\frac{19}{20}-\varepsilon}$, providing $M$ and $H$ satisfy the following conditions:

$$
\begin{gathered}
M H \ll X^{\frac{25}{44}}, \quad M^{35} H^{23} \ll X^{\frac{179}{10}}, \quad X^{\frac{19}{22}} \ll M^{2} H, \\
M^{2} H^{13} \ll X^{\frac{31}{10}}, \quad X^{\frac{171}{5}} \ll M^{70} H^{59} .
\end{gathered}
$$

We apply the mean value estimate and Halász method to $M^{2}(s) H(s)$, $H^{6}(s)$ and $K^{2}(s)$ to get

$$
I \ll U^{2} V^{2} W^{2} x^{-1} F \log ^{c} x
$$

where

$$
\begin{aligned}
F=\min \{ & V^{-4} W^{-2}\left(M^{2} H+T\right), V^{-4} W^{-2} M^{2} H+V^{-12} W^{-6} M^{2} H T, \\
& W^{-12}\left(H^{6}+T\right), W^{-12} H^{6}+W^{-36} H^{6} T, U^{-4}\left(K^{2}+T\right), \\
& \left.U^{-4} K^{2}+U^{-12} K^{2} T\right\} .
\end{aligned}
$$

Consider the following cases:

(a) $F \leq 2 V^{-4} W^{-2} M^{2} H, 2 W^{-12} H^{6}, 2 U^{-4} K^{2}$. Then

$$
\begin{aligned}
U^{2} V^{2} W^{2} F & \ll U^{2} V^{2} W^{2} \min \left\{V^{-4} W^{-2} M^{2} H, W^{-12} H^{6}, U^{-4} K^{2}\right\} \\
& \leq U^{2} V^{2} W^{2}\left(V^{-4} W^{-2} M^{2} H\right)^{\frac{1}{2}}\left(U^{-4} K^{2}\right)^{\frac{1}{2}} \\
& =W H^{\frac{1}{2}} M K \ll x \log ^{-11 B} x .
\end{aligned}
$$


(b) $F \leq 2 V^{-4} W^{-2} M^{2} H, 2 W^{-12} H^{6}, F>2 U^{-4} K^{2}$. Then

$$
\begin{aligned}
& U^{2} V^{2} W^{2} F \\
& \quad \ll U^{2} V^{2} W^{2} \min \left\{V^{-4} W^{-2} M^{2} H, W^{-12} H^{6}, U^{-4} T, U^{-12} K^{2} T\right\} \\
& \quad \leq U^{2} V^{2} W^{2}\left(V^{-4} W^{-2} M^{2} H\right)^{\frac{1}{2}}\left(W^{-12} H^{6}\right)^{\frac{1}{12}}\left(U^{-4} T\right)^{\frac{3}{8}}\left(U^{-12} K^{2} T\right)^{\frac{1}{24}} \\
& \quad=T^{\frac{5}{12}} M H K^{\frac{1}{12}} \ll x^{1-\varepsilon_{1}} .
\end{aligned}
$$

(c) $F \leq 2 V^{-4} W^{-2} M^{2} H, F>2 W^{-12} H^{6}, F \leq 2 U^{-4} K^{2}$. Then

$$
\begin{aligned}
U^{2} V^{2} W^{2} F & \ll U^{2} V^{2} W^{2} \min \left\{V^{-4} W^{-2} M^{2} H, W^{-12} T, W^{-36} H^{6} T, U^{-4} K^{2}\right\} \\
& \leq U^{2} V^{2} W^{2}\left(V^{-4} W^{-2} M^{2} H\right)^{\frac{1}{2}}\left(U^{-4} K^{2}\right)^{\frac{1}{2}} \\
& =W H^{\frac{1}{2}} M K \ll x \log ^{-11 B} x .
\end{aligned}
$$

(d) $F \leq 2 V^{-4} W^{-2} M^{2} H, F>2 W^{-12} H^{6}, 2 U^{-4} K^{2}$. Then

$$
\begin{aligned}
& U^{2} V^{2} W^{2} F \\
& \ll U^{2} V^{2} W^{2} \min \left\{V^{-4} W^{-2} M^{2} H, W^{-12} T, W^{-36} H^{6} T, U^{-4} T, U^{-12} K^{2} T\right\} \\
& \leq U^{2} V^{2} W^{2}\left(V^{-4} W^{-2} M^{2} H\right)^{\frac{1}{2}}\left(W^{-36} H^{6} T\right)^{\frac{1}{36}}\left(U^{-4} T\right)^{\frac{11}{24}}\left(U^{-12} K^{2} T\right)^{\frac{1}{72}} \\
& =T^{\frac{1}{2}} M H^{\frac{2}{3}} K^{\frac{1}{36}} \ll x^{1-\varepsilon_{1}} .
\end{aligned}
$$

(e) $F>2 V^{-4} W^{-2} M^{2} H, F \leq 2 W^{-12} H^{6}, 2 U^{-4} K^{2}$. Then

$$
\begin{aligned}
& U^{2} V^{2} W^{2} F \\
& \ll U^{2} V^{2} W^{2} \min \left\{V^{-4} W^{-2} T, V^{-12} W^{-6} M^{2} H T, W^{-12} H^{6}, U^{-4} K^{2}\right\} \\
& \leq U^{2} V^{2} W^{2}\left(V^{-4} W^{-2} T\right)^{\frac{3}{8}}\left(V^{-12} W^{-6} M^{2} H T\right)^{\frac{1}{24}}\left(W^{-12} H^{6}\right)^{\frac{1}{12}}\left(U^{-4} K^{2}\right)^{\frac{1}{2}} \\
& =T^{\frac{5}{12}} M^{\frac{1}{12}} H^{\frac{13}{24}} K \ll x^{1-\varepsilon_{1}} .
\end{aligned}
$$

(f) $F>2 V^{-4} W^{-2} M^{2} H, F \leq 2 W^{-12} H^{6}, F>2 U^{-4} K^{2}$. Then

$$
\begin{aligned}
& U^{2} V^{2} W^{2} F \\
& \ll U^{2} V^{2} W^{2} \min \left\{V^{-4} W^{-2} T, V^{-12} W^{-6} M^{2} H T, W^{-12} H^{6},\right. \\
& \left.\quad U^{-4} T, U^{-12} K^{2} T\right\} \\
& \leq U^{2} V^{2} W^{2}\left(V^{-4} W^{-2} T\right)^{\frac{3}{8}}\left(V^{-12} W^{-6} M^{2} H T\right)^{\frac{1}{24}}\left(W^{-12} H^{6}\right)^{\frac{1}{12}}\left(U^{-4} T\right)^{\frac{1}{2}} \\
& =T^{\frac{11}{12}} M^{\frac{1}{12}} H^{\frac{13}{24}} \ll x^{1-\varepsilon_{1}} .
\end{aligned}
$$


(g) $F>2 V^{-4} W^{-2} M^{2} H, 2 W^{-12} H^{6}, F \leq 2 U^{-4} K^{2}$. Then

$$
\begin{aligned}
& U^{2} V^{2} W^{2} F \\
& \ll U^{2} V^{2} W^{2} \min \left\{V^{-4} W^{-2} T, V^{-12} W^{-6} M^{2} H T, W^{-12} T,\right. \\
& \left.\quad W^{-36} H^{6} T, U^{-4} K^{2}\right\} \\
& \leq U^{2} V^{2} W^{2}\left(V^{-4} W^{-2} T\right)^{\frac{11}{24}}\left(V^{-12} W^{-6} M^{2} H T\right)^{\frac{1}{72}}\left(W^{-36} H^{6} T\right)^{\frac{1}{36}}\left(U^{-4} K^{2}\right)^{\frac{1}{2}} \\
& =T^{\frac{1}{2}} M^{\frac{1}{36}} H^{\frac{13}{72}} K \ll x^{1-\varepsilon_{1}} .
\end{aligned}
$$

(h) $F>2 V^{-4} W^{-2} M^{2} H, 2 W^{-12} H^{6}, 2 U^{-4} K^{2}$. Then

$$
\begin{aligned}
U^{2} V^{2} W^{2} F \ll & U^{2} V^{2} W^{2} \min \left\{V^{-4} W^{-2}, V^{-12} W^{-6} M^{2} H, W^{-12}, W^{-36} H^{6},\right. \\
& \left.U^{-4}, U^{-12} K^{2}\right\} T \\
\leq & U^{2} V^{2} W^{2}\left(V^{-4} W^{-2}\right)^{\frac{1}{2}}\left(W^{-12}\right)^{\frac{1}{12}}\left(U^{-4}\right)^{\frac{3}{8}}\left(U^{-12} K^{2}\right)^{\frac{1}{24}} T \\
& =T K^{\frac{1}{12}} \ll x^{1-\varepsilon_{1}},
\end{aligned}
$$

since $X^{\frac{2}{5}} \ll M H$ (the latter follows from $X^{\frac{171}{5}} \ll M^{70} H^{59}$ ).

In every region, our conditions are satisfied. So the proof of Lemma 9 is complete.

Lemma 10. Under the assumption of Lemma 5, suppose that $M$ and $H$ lie in one of the following regions:

(i) $\quad X^{\frac{37}{100}} \ll M \ll X^{\frac{5541}{13940}}, \quad M^{-\frac{82}{69}} X^{\frac{133}{230}} \ll H \ll M^{-\frac{70}{59}} X^{\frac{171}{295}}$;

(ii) $\quad X^{\frac{5541}{13940}} \ll M \ll X^{\frac{1188}{2975}}, \quad X^{\frac{9}{85}} \ll H \ll M^{-\frac{70}{59}} X^{\frac{171}{295}}$;

(iii) $\quad X^{\frac{19}{45}} \ll M \ll X^{\frac{4331}{9860}}, \quad M^{-\frac{35}{23}} X^{\frac{179}{230}} \ll H \ll M^{-\frac{41}{27}} X^{\frac{421}{540}}$.

Then (7) holds for $T_{0} \leq T \leq X$.

Proof. First we show that (8) holds for $T=1 / \eta=2 X^{\frac{19}{20}-\varepsilon}$, providing $M$ and $H$ satisfy the following conditions:

$$
\begin{gathered}
M H \ll X^{\frac{73}{130}}, \quad M^{41} H^{27} \ll X^{\frac{421}{20}}, \quad X^{\frac{57}{65}} \ll M^{2} H, \\
M^{2} H^{15} \ll X^{\frac{33}{10}}, \quad X^{\frac{399}{10}} \ll M^{82} H^{69} .
\end{gathered}
$$

We apply the mean value estimate and Halász method to $M^{2}(s) H(s)$, $H^{7}(s)$ and $K^{2}(s)$ to get

$$
I \ll U^{2} V^{2} W^{2} x^{-1} F \log ^{c} x,
$$

where

$$
\begin{aligned}
F=\min \{ & V^{-4} W^{-2}\left(M^{2} H+T\right), V^{-4} W^{-2} M^{2} H+V^{-12} W^{-6} M^{2} H T, \\
& W^{-14}\left(H^{7}+T\right), W^{-14} H^{7}+W^{-42} H^{7} T, U^{-4}\left(K^{2}+T\right), \\
& \left.U^{-4} K^{2}+U^{-12} K^{2} T\right\} .
\end{aligned}
$$

Consider the following cases: 
(a) $F \leq 2 V^{-4} W^{-2} M^{2} H, 2 W^{-14} H^{7}, 2 U^{-4} K^{2}$. Then

$$
\begin{aligned}
U^{2} V^{2} W^{2} F & \ll U^{2} V^{2} W^{2} \min \left\{V^{-4} W^{-2} M^{2} H, W^{-14} H^{7}, U^{-4} K^{2}\right\} \\
& \leq U^{2} V^{2} W^{2}\left(V^{-4} W^{-2} M^{2} H\right)^{\frac{1}{2}}\left(U^{-4} K^{2}\right)^{\frac{1}{2}} \\
& =W H^{\frac{1}{2}} M K \ll x \log ^{-11 B} x .
\end{aligned}
$$

(b) $F \leq 2 V^{-4} W^{-2} M^{2} H, 2 W^{-14} H^{7}, F>2 U^{-4} K^{2}$. Then

$$
\begin{aligned}
U^{2} V^{2} & W^{2} F \\
& \ll U^{2} V^{2} W^{2} \min \left\{V^{-4} W^{-2} M^{2} H, W^{-14} H^{7}, U^{-4} T, U^{-12} K^{2} T\right\} \\
& \leq U^{2} V^{2} W^{2}\left(V^{-4} W^{-2} M^{2} H\right)^{\frac{1}{2}}\left(W^{-14} H^{7}\right)^{\frac{1}{14}}\left(U^{-4} T\right)^{\frac{11}{28}}\left(U^{-12} K^{2} T\right)^{\frac{1}{28}} \\
& =T^{\frac{3}{7}} M H K^{\frac{1}{14}} \ll x^{1-\varepsilon_{1}} .
\end{aligned}
$$

(c) $F \leq 2 V^{-4} W^{-2} M^{2} H, F>2 W^{-14} H^{7}, F \leq 2 U^{-4} K^{2}$. Then

$$
\begin{aligned}
& U^{2} V^{2} W^{2} F \ll U^{2} V^{2} W^{2} \min \left\{V^{-4} W^{-2} M^{2} H, W^{-14} T, W^{-42} H^{7} T, U^{-4} K^{2}\right\} \\
& \quad \leq U^{2} V^{2} W^{2}\left(V^{-4} W^{-2} M^{2} H\right)^{\frac{1}{2}}\left(U^{-4} K^{2}\right)^{\frac{1}{2}} \\
& \quad=W H^{\frac{1}{2}} M K \ll x \log { }^{-11 B} x . \\
& \text { (d) } F \leq 2 V^{-4} W^{-2} M^{2} H, F>2 W^{-14} H^{7}, 2 U^{-4} K^{2} . \text { Then } \\
& U^{2} V^{2} W^{2} F \\
& \ll U^{2} V^{2} W^{2} \min \left\{V^{-4} W^{-2} M^{2} H, W^{-14} T, W^{-42} H^{7} T, U^{-4} T, U^{-12} K^{2} T\right\} \\
& \leq U^{2} V^{2} W^{2}\left(V^{-4} W^{-2} M^{2} H\right)^{\frac{1}{2}}\left(W^{-42} H^{7} T\right)^{\frac{1}{42}}\left(U^{-4} T\right)^{\frac{13}{28}}\left(U^{-12} K^{2} T\right)^{\frac{1}{84}} \\
& =T^{\frac{1}{2}} M H^{\frac{2}{3}} K^{\frac{1}{42}} \ll x^{1-\varepsilon_{1}} . \\
& \left(\text { e) } F>2 V^{-4} W^{-2} M^{2} H, F \leq 2 W^{-14} H^{7}, 2 U^{-4} K^{2} .\right. \text { Then } \\
& U^{2} V^{2} W^{2} F \\
& \ll U^{2} V^{2} W^{2} \min \left\{V^{-4} W^{-2} T, V^{-12} W^{-6} M^{2} H T, W^{-14} H^{7}, U^{-4} K^{2}\right\} \\
& \leq U^{2} V^{2} W^{2}\left(V^{-4} W^{-2} T\right)^{\frac{11}{28}}\left(V^{-12} W^{-6} M^{2} H T\right)^{\frac{1}{28}}\left(W^{-14} H^{7}\right)^{\frac{1}{14}}\left(U^{-4} K^{2}\right)^{\frac{1}{2}} \\
& =T^{\frac{3}{7}} M^{\frac{1}{14}} H^{\frac{15}{28}} K \ll x^{1-\varepsilon_{1}} . \\
& \text { (f) } F>2 V^{-4} W^{-2} M^{2} H, F \leq 2 W^{-14} H^{7}, F>2 U^{-4} K^{2} . \text { Then } \\
& U^{2} V^{2} W^{2} F \\
& \ll U^{2} V^{2} W^{2} \min \left\{V^{-4} W^{-2} T, V^{-12} W^{-6} M^{2} H T, W^{-14} H^{7},\right. \\
& \left.\quad U^{-4} T, U^{-12} K^{2} T\right\} \\
& \leq U^{2} V^{2} W^{2}\left(V^{-4} W^{-2} T\right)^{\frac{11}{28}}\left(V^{-12} W^{-6} M^{2} H T\right)^{\frac{1}{28}}\left(W^{-14} H^{7}\right)^{\frac{1}{14}}\left(U^{-4} T\right)^{\frac{1}{2}} \\
& =T^{13} M^{\frac{1}{14}} H^{\frac{15}{28}} \ll x^{1-\varepsilon_{1}} .
\end{aligned}
$$


(g) $F>2 V^{-4} W^{-2} M^{2} H, 2 W^{-14} H^{7}, F \leq 2 U^{-4} K^{2}$. Then

$$
\begin{aligned}
& U^{2} V^{2} W^{2} F \ll U^{2} V^{2} W^{2} \min \left\{V^{-4} W^{-2} T, V^{-12} W^{-6} M^{2} H T, W^{-14} T,\right. \\
&\left.W^{-42} H^{7} T, U^{-4} K^{2}\right\} \\
& \leq U^{2} V^{2} W^{2}\left(V^{-4} W^{-2} T\right)^{\frac{13}{28}}\left(V^{-12} W^{-6} M^{2} H T\right)^{\frac{1}{84}} \\
& \times\left(W^{-42} H^{7} T\right)^{\frac{1}{42}}\left(U^{-4} K^{2}\right)^{\frac{1}{2}} \\
&= T^{\frac{1}{2}} M^{\frac{1}{42}} H^{\frac{5}{28}} K \ll x^{1-\varepsilon_{1}} .
\end{aligned}
$$

(h) $F>2 V^{-4} W^{-2} M^{2} H, 2 W^{-14} H^{7}, 2 U^{-4} K^{2}$. Then

$$
\begin{aligned}
U^{2} V^{2} W^{2} F & \ll U^{2} V^{2} W^{2} \min \left\{V^{-4} W^{-2}, V^{-12} W^{-6} M^{2} H, W^{-14}, W^{-42} H^{7},\right. \\
& \left.U^{-4}, U^{-12} K^{2}\right\} T \\
& \leq U^{2} V^{2} W^{2}\left(V^{-4} W^{-2}\right)^{\frac{1}{2}}\left(W^{-14}\right)^{\frac{1}{14}}\left(U^{-4}\right)^{\frac{11}{28}}\left(U^{-12} K^{2}\right)^{\frac{1}{28}} T \\
& =T K^{\frac{1}{14}} \ll x^{1-\varepsilon_{1}},
\end{aligned}
$$

since $X^{\frac{3}{10}} \ll M H$ (the latter follows from $X^{\frac{57}{65}} \ll M^{2} H$ ).

In every region, our conditions are satisfied, so the proof of Lemma 10 is complete.

Lemma 11. Under the assumption of Lemma 5, suppose that $M$ and $H$ lie in one of the following regions:

(i) $\quad X^{\frac{1843}{5280}} \ll M \ll X^{\frac{37}{100}}, \quad M^{-\frac{70}{59}} X^{\frac{171}{295}} \ll H \ll M^{-\frac{58}{49}} X^{\frac{57}{98}}$;

(ii) $\quad X^{\frac{37}{100}} \ll M \ll X^{\frac{5541}{13940}}, \quad M^{-\frac{82}{69}} X^{\frac{133}{230}} \ll H \ll M^{-\frac{58}{49}} X^{\frac{57}{98}}$;

(iii) $\quad X^{\frac{5541}{13940}} \ll M \ll X^{\frac{3963}{9860}}, \quad X^{\frac{9}{85}} \ll H \ll M^{-\frac{58}{49}} X^{\frac{57}{98}}$;

(iv) $\quad X^{\frac{1063}{2640}} \ll M \ll X^{\frac{19}{45}}, \quad M^{-\frac{29}{19}} X^{\frac{59}{76}} \ll H \ll M^{-\frac{35}{23}} X^{\frac{179}{230}}$;

(v) $\quad X^{\frac{19}{45}} \ll M \ll X^{\frac{4331}{9860}}, \quad M^{-\frac{29}{19}} X^{\frac{59}{76}} \ll H \ll M^{-\frac{41}{27}} X^{\frac{421}{540}}$.

Then (7) holds for $T_{0} \leq T \leq X$.

Pr o of. Putting together regions in Lemmas 9 and 10, we can get Lemma 11.

LEMMA 12. Under the assumption of Lemma 5, suppose that $M$ and $H$ lie in one of the following regions:

(i) $\quad X^{\frac{4331}{9860}} \ll M \ll X^{\frac{2691}{5950}}, \quad M^{-\frac{35}{12}} X^{\frac{57}{40}} \ll H \ll M^{-\frac{29}{10}} X^{\frac{57}{40}}$;

(ii) $\quad X^{\frac{2691}{5950}} \ll M \ll X^{\frac{897}{1972}}, \quad X^{\frac{9}{85}} \ll H \ll M^{-\frac{29}{10}} X^{\frac{57}{40}}$;

(iii) $\quad X^{\frac{233}{480}} \ll M \ll X^{\frac{49}{100}}, \quad M^{-\frac{58}{9}} X^{\frac{59}{18}} \ll H \ll M^{-\frac{70}{11}} X^{\frac{179}{55}}$;

(iv) $\quad X^{\frac{49}{100}} \ll M \ll X^{\frac{2944}{5950}}, \quad X^{\frac{9}{85}} \ll H \ll M^{-\frac{70}{11}} X^{\frac{179}{55}}$.

Then (7) holds for $T_{0} \leq T \leq X$. 
Proof. First we show that (8) holds for $T=1 / \eta=2 X^{\frac{19}{20}-\varepsilon}$, providing that $M$ and $H$ satisfy the following conditions:

$$
\begin{gathered}
M^{2} H \ll X^{\frac{25}{22}}, \quad M^{70} H^{11} \ll X^{\frac{179}{5}}, \quad X^{\frac{19}{44}} \ll M, \\
M H^{6} \ll X^{\frac{31}{20}}, \quad X^{\frac{171}{10}} \ll M^{35} H^{12} .
\end{gathered}
$$

We apply the mean value estimate and Halász method to $M^{2}(s), H^{6}(s)$ and $K^{2}(s) H(s)$ to get

$$
I \ll U^{2} V^{2} W^{2} x^{-1} F \log ^{c} x,
$$

where

$$
\begin{aligned}
F=\min \{ & V^{-4}\left(M^{2}+T\right), V^{-4} M^{2}+V^{-12} M^{2} T, W^{-12}\left(H^{6}+T\right), W^{-12} H^{6} \\
& +W^{-36} H^{6} T, U^{-4} W^{-2}\left(K^{2} H+T\right), U^{-4} W^{-2} K^{2} H \\
& \left.+U^{-12} W^{-6} K^{2} H T\right\} .
\end{aligned}
$$

Consider the following cases:

(a) $F \leq 2 V^{-4} M^{2}, 2 W^{-12} H^{6}, 2 U^{-4} W^{-2} K^{2} H$. Then

$$
\begin{aligned}
U^{2} V^{2} W^{2} F & \ll U^{2} V^{2} W^{2} \min \left\{V^{-4} M^{2}, W^{-12} H^{6}, U^{-4} W^{-2} K^{2} H\right\} \\
& \leq U^{2} V^{2} W^{2}\left(V^{-4} M^{2}\right)^{\frac{1}{2}}\left(U^{-4} W^{-2} K^{2} H\right)^{\frac{1}{2}} \\
& =W H^{\frac{1}{2}} M K \ll x \log ^{-11 B} x .
\end{aligned}
$$

(b) $F \leq 2 V^{-4} M^{2}, 2 W^{-12} H^{6}, F>2 U^{-4} W^{-2} K^{2} H$. Then

$$
\begin{aligned}
& U^{2} V^{2} W^{2} F \\
& \ll U^{2} V^{2} W^{2} \min \left\{V^{-4} M^{2}, W^{-12} H^{6}, U^{-4} W^{-2} T, U^{-12} W^{-6} K^{2} H T\right\} \\
& \leq U^{2} V^{2} W^{2}\left(V^{-4} M^{2}\right)^{\frac{1}{2}}\left(W^{-12} H^{6}\right)^{\frac{1}{12}}\left(U^{-4} W^{-2} T\right)^{\frac{3}{8}}\left(U^{-12} W^{-6} K^{2} H T\right)^{\frac{1}{24}} \\
& =T^{\frac{5}{12}} M H^{\frac{13}{24}} K^{\frac{1}{12}} \ll x^{1-\varepsilon_{1}} \text {. } \\
& \text { (c) } F \leq 2 V^{-4} M^{2}, F>2 W^{-12} H^{6}, F \leq 2 U^{-4} W^{-2} K^{2} H \text {. Then } \\
& U^{2} V^{2} W^{2} F \ll U^{2} V^{2} W^{2} \min \left\{V^{-4} M^{2}, W^{-12} T, W^{-36} H^{6} T, U^{-4} W^{-2} K^{2} H\right\} \\
& \leq U^{2} V^{2} W^{2}\left(V^{-4} M^{2}\right)^{\frac{1}{2}}\left(U^{-4} W^{-2} K^{2} H\right)^{\frac{1}{2}} \\
& =W H^{\frac{1}{2}} M K \ll x \log ^{-11 B} x \text {. } \\
& \text { (d) } F \leq 2 V^{-4} M^{2}, F>2 W^{-12} H^{6}, 2 U^{-4} W^{-2} K^{2} H \text {. Then } \\
& U^{2} V^{2} W^{2} F \ll U^{2} V^{2} W^{2} \min \left\{V^{-4} M^{2}, W^{-12} T, W^{-36} H^{6} T,\right. \\
& \left.U^{-4} W^{-2} T, U^{-12} W^{-6} K^{2} H T\right\} \\
& \leq U^{2} V^{2} W^{2}\left(V^{-4} M^{2}\right)^{\frac{1}{2}}\left(W^{-36} H^{6} T\right)^{\frac{1}{36}}\left(U^{-4} W^{-2} T\right)^{\frac{11}{24}} \\
& \times\left(U^{-12} W^{-6} K^{2} H T\right)^{\frac{1}{72}} \\
& =T^{\frac{1}{2}} M H^{\frac{13}{72}} K^{\frac{1}{36}} \ll x^{1-\varepsilon_{1}} \text {. }
\end{aligned}
$$


(e) $F>2 V^{-4} M^{2}, F \leq 2 W^{-12} H^{6}, 2 U^{-4} W^{-2} K^{2} H$. Then

$$
\begin{aligned}
U^{2} V^{2} & W^{2} F \\
& \ll U^{2} V^{2} W^{2} \min \left\{V^{-4} T, V^{-12} M^{2} T, W^{-12} H^{6}, U^{-4} W^{-2} K^{2} H\right\} \\
& \leq U^{2} V^{2} W^{2}\left(V^{-4} T\right)^{\frac{3}{8}}\left(V^{-12} M^{2} T\right)^{\frac{1}{24}}\left(W^{-12} H^{6}\right)^{\frac{1}{12}}\left(U^{-4} W^{-2} K^{2} H\right)^{\frac{1}{2}} \\
& =T^{\frac{5}{12}} M^{\frac{1}{12}} H K \ll x^{1-\varepsilon_{1}} .
\end{aligned}
$$

(f) $F>2 V^{-4} M^{2}, F \leq 2 W^{-12} H^{6}, F>2 U^{-4} W^{-2} K^{2} H$. Then

$$
\begin{aligned}
U^{2} V^{2} W^{2} F \ll & U^{2} V^{2} W^{2} \min \left\{V^{-4} T, V^{-12} M^{2} T, W^{-12} H^{6}, U^{-4} W^{-2} T,\right. \\
& \left.U^{-12} W^{-6} K^{2} H T\right\} \\
\leq & U^{2} V^{2} W^{2}\left(V^{-4} T\right)^{\frac{3}{8}}\left(V^{-12} M^{2} T\right)^{\frac{1}{24}}\left(W^{-12} H^{6}\right)^{\frac{1}{12}}\left(U^{-4} W^{-2} T\right)^{\frac{1}{2}} \\
= & T^{\frac{11}{12}} M^{\frac{1}{12}} H^{\frac{1}{2}} \ll x^{1-\varepsilon_{1}} .
\end{aligned}
$$

(g) $F>2 V^{-4} M^{2}, 2 W^{-12} H^{6}, F \leq 2 U^{-4} W^{-2} K^{2} H$. Then

$$
\begin{aligned}
& U^{2} V^{2} W^{2} F \\
& \ll U^{2} V^{2} W^{2} \min \left\{V^{-4} T, V^{-12} M^{2} T, W^{-12} T, W^{-36} H^{6} T, U^{-4} W^{-2} K^{2} H\right\} \\
& \leq U^{2} V^{2} W^{2}\left(V^{-4} T\right)^{\frac{11}{24}}\left(V^{-12} M^{2} T\right)^{\frac{1}{72}}\left(W^{-36} H^{6} T\right)^{\frac{1}{36}}\left(U^{-4} W^{-2} K^{2} H\right)^{\frac{1}{2}} \\
& =T^{\frac{1}{2}} M^{\frac{1}{36}} H^{\frac{2}{3}} K \ll x^{1-\varepsilon_{1}} .
\end{aligned}
$$

(h) $F>2 V^{-4} M^{2}, 2 W^{-12} H^{6}, 2 U^{-4} W^{-2} K^{2} H$. Then

$$
\begin{aligned}
U^{2} V^{2} W^{2} F & \ll U^{2} V^{2} W^{2} \min \left\{V^{-4}, V^{-12} M^{2}, W^{-12}, W^{-36} H^{6}, U^{-4} W^{-2},\right. \\
& \left.U^{-12} W^{-6} K^{2} H\right\} T \\
& \leq U^{2} V^{2} W^{2}\left(V^{-4}\right)^{\frac{3}{8}}\left(V^{-12} M^{2}\right)^{\frac{1}{24}}\left(W^{-12}\right)^{\frac{1}{12}}\left(U^{-4} W^{-2}\right)^{\frac{1}{2}} T \\
& =T M^{\frac{1}{12}} \ll x^{1-\varepsilon_{1}},
\end{aligned}
$$

since $M \ll X^{\frac{3}{5}}$ (the latter follows from $M^{70} H^{11} \ll X^{\frac{179}{5}}$ ).

In every region, our conditions are satisfied. So the proof of Lemma 12 is complete.

\section{Mean value estimate (III)}

Lemma 13. Suppose that $P Q R K=X$ and that $P(s), Q(s), R(s)$ and $K(s)$ are Dirichlet polynomials. Define $G(s)=P(s) Q(s) R(s) K(s)$. Let $b=$ $1+1 / \log X$ and $T_{0}=\log ^{\frac{B}{\varepsilon}} X$. Assume further that for $T_{0} \leq|t| \leq 2 X$, $P(b+i t) Q(b+i t) \ll \log ^{-\frac{B}{\varepsilon}} x$ and $R(b+i t) \ll \log ^{-\frac{B}{\varepsilon}} x$. Moreover, assume that

$$
X^{\frac{9}{85}} \ll R \ll Q
$$


and that $P$ and $Q$ lie in one of the following regions:

(ii) $\quad X^{\frac{19}{80}} \ll P \ll X^{\frac{53}{220}}, \quad P^{-1} X^{\frac{133}{290}} \ll Q \ll P^{-1} X^{\frac{19}{40}}$;

(iii) $\quad X^{\frac{53}{220}} \ll P \ll X^{\frac{151}{580}}, \quad P^{-1} X^{\frac{133}{290}} \ll Q \ll P^{-1} X^{\frac{53}{110}}$;

(iv) $\quad X^{\frac{151}{580}} \ll P \ll X^{\frac{17}{55}}, \quad P^{-1} X^{\frac{897}{1972}} \ll Q \ll P^{-1} X^{\frac{53}{110}}$;

(v) $\quad X^{\frac{17}{55}} \ll P \ll X^{\frac{3441}{9860}}, \quad P^{-1} X^{\frac{897}{1972}} \ll Q \ll P^{-\frac{58}{67}} X^{\frac{59}{134}}$;

(vi) $\quad X^{\frac{3441}{9860}} \ll P \ll X^{\frac{37}{100}}, \quad X^{\frac{9}{85}} \ll Q \ll P^{-\frac{58}{67}} X^{\frac{59}{134}}$;

(vii) $\quad X^{\frac{37}{100}} \ll P \ll X^{\frac{653}{1700}}, \quad X^{\frac{9}{85}} \ll Q \ll P^{-1} X^{\frac{49}{100}}$.

Then (7) holds for $T_{0} \leq T \leq X$.

Proof. Let $m=p q, h=r$.

(a) On applying Lemma 8 with region (iv), we see that (7) holds under the conditions

$$
P^{-1} X^{\frac{897}{1972}} \ll Q \ll P^{-1} X^{\frac{133}{290}}, \quad X^{\frac{9}{85}} \ll R \ll Q \ll(P Q)^{-\frac{1}{5}} X^{\frac{29}{100}},
$$

which can be written as

$$
P^{-1} X^{\frac{897}{1972}} \ll Q \ll P^{-1} X^{\frac{133}{290}}, \quad X^{\frac{9}{85}} \ll Q \ll P^{-\frac{1}{6}} X^{\frac{29}{120}}, \quad X^{\frac{9}{85}} \ll R \ll Q .
$$

In the regions:

$$
\begin{array}{ll}
X^{\frac{151}{580}} \ll P \ll X^{\frac{3441}{9860}}, & P^{-1} X^{\frac{897}{1972}} \ll Q \ll P^{-1} X^{\frac{133}{290}} ; \\
X^{\frac{3441}{9860}} \ll P \ll X^{\frac{1739}{4930}}, & X^{\frac{9}{85}} \ll Q \ll P^{-1} X^{\frac{133}{290}},
\end{array}
$$

the above conditions on $P$ and $Q$ are satisfied.

(b) On applying Lemma 8 with region (vi), we see that (7) holds under the conditions

$$
P^{-1} X^{\frac{133}{290}} \ll Q \ll P^{-1} X^{\frac{19}{40}}, \quad X^{\frac{9}{85}} \ll R \ll Q \ll(P Q)^{\frac{1}{7}} X^{\frac{13}{70}},
$$

which can be written as

$$
P^{-1} X^{\frac{133}{290}} \ll Q \ll P^{-1} X^{\frac{19}{40}}, \quad X^{\frac{9}{85}} \ll Q \ll P^{\frac{1}{6}} X^{\frac{13}{60}}, \quad X^{\frac{9}{85}} \ll R \ll Q .
$$

In the regions:

$$
\begin{aligned}
& X^{\frac{133}{580}} \ll P \ll X^{\frac{19}{80}}, \quad P^{-1} X^{\frac{133}{290}} \ll Q \ll P ; \\
& X^{\frac{19}{80}} \ll P \ll X^{\frac{1739}{4930}}, \quad P^{-1} X^{\frac{133}{290}} \ll Q \ll P^{-1} X^{\frac{19}{40}} ; \\
& X^{\frac{1739}{4930}} \ll P \ll X^{\frac{251}{680}}, \quad X^{\frac{9}{85}} \ll Q \ll P^{-1} X^{\frac{19}{40}},
\end{aligned}
$$

the above conditions on $P$ and $Q$ are satisfied.

(c) On applying Lemma 8 with region (vii), we see that (7) holds under the conditions

$$
P^{-1} X^{\frac{19}{40}} \ll Q \ll P^{-1} X^{\frac{53}{110}}, \quad X^{\frac{9}{85}} \ll R \ll Q \ll(P Q)^{-1} X^{\frac{51}{70}},
$$


which can be written as

$$
P^{-1} X^{\frac{19}{40}} \ll Q \ll P^{-1} X^{\frac{53}{110}}, \quad X^{\frac{9}{85}} \ll Q \ll P^{-\frac{1}{2}} X^{\frac{51}{140}}, \quad X^{\frac{9}{85}} \ll R \ll Q .
$$

In the regions:

$$
\begin{array}{ll}
X^{\frac{53}{220}} \ll P \ll X^{\frac{251}{680}}, & P^{-1} X^{\frac{19}{40}} \ll Q \ll P^{-1} X^{\frac{53}{110}} ; \\
X^{\frac{251}{680}} \ll P \ll X^{\frac{703}{1870}}, & X^{\frac{9}{85}} \ll Q \ll P^{-1} X^{\frac{53}{110}},
\end{array}
$$

the above conditions on $P$ and $Q$ are satisfied.

(d) On applying Lemma 8 with regions (viii) and (x), we see that (7) holds under the conditions

$$
P^{-1} X^{\frac{53}{110}} \ll Q \ll P^{-1} X^{\frac{49}{100}}, \quad X^{\frac{9}{85}} \ll R \ll Q \ll(P Q)^{-\frac{58}{9}} X^{\frac{59}{18}},
$$

which can be written as

$$
P^{-1} X^{\frac{53}{110}} \ll Q \ll P^{-1} X^{\frac{49}{100}}, \quad X^{\frac{9}{85}} \ll Q \ll P^{-\frac{58}{67}} X^{\frac{59}{134}}, \quad X^{\frac{9}{85}} \ll R \ll Q .
$$

In the regions:

$$
\begin{aligned}
& X^{\frac{17}{55}} \ll P \ll X^{\frac{37}{100}}, \quad P^{-1} X^{\frac{53}{110}} \ll Q \ll P^{-\frac{58}{67}} X^{\frac{59}{134}} ; \\
& X^{\frac{37}{100}} \ll P \ll X^{\frac{703}{1870}}, \quad P^{-1} X^{\frac{53}{110}} \ll Q \ll P^{-1} X^{\frac{49}{100}} ; \\
& X^{\frac{703}{1870}} \ll P \ll X^{\frac{653}{1700}}, \quad X^{\frac{9}{85}} \ll Q \ll P^{-1} X^{\frac{49}{100}},
\end{aligned}
$$

the above conditions on $P$ and $Q$ are satisfied.

Putting together the above regions, we get Lemma 13.

Lemma 14. Suppose that $P Q R L=X$ and that $P(s), Q(s)$ and $R(s)$ are Dirichlet polynomials,

$$
L(s)=\sum_{l \sim L} \frac{1}{l^{s}},
$$

and $F(s)=P(s) Q(s) R(s) L(s)$. Let $b=1+1 / \log X$ and $T_{1}=\sqrt{L}$. Assume further that for $T_{1} \leq|t| \leq 2 X, P(b+i t) Q(b+i t) \ll \log ^{-\frac{B}{\varepsilon}} x$ and $R(b+i t) \ll$ $\log ^{-\frac{B}{\varepsilon}} x$. Moreover, assume that

$$
X^{\frac{9}{85}} \ll R \ll Q
$$

and that $P$ and $Q$ lie in one of the following regions:

$$
\begin{aligned}
& X^{\frac{9}{85}} \ll P \ll X^{\frac{21}{100}}, \quad X^{\frac{9}{85}} \ll Q \ll P ; \\
& X^{\frac{21}{100}} \ll P \ll X^{\frac{13}{60}}, \quad X^{\frac{9}{85}} \ll Q \ll P^{-\frac{2}{3}} X^{\frac{7}{20}} ; \\
& X^{\frac{13}{60}} \ll P \ll X^{\frac{12613}{49300}}, \quad X^{\frac{9}{85}} \ll Q \ll P^{-\frac{1}{6}} X^{\frac{29}{120}} ; \\
& X^{\frac{12613}{49300}} \ll P \ll X^{\frac{3441}{9860}}, \quad X^{\frac{9}{85}} \ll Q \ll P^{-1} X^{\frac{897}{1972}} \text {. }
\end{aligned}
$$


Then for $T_{1} \leq T \leq X$, we have

$$
\min ^{2}\left(\eta, \frac{1}{T}\right) \int_{T}^{2 T}|F(b+i t)|^{2} d t \ll \eta^{2} \log ^{-10 B} x .
$$

Proof. Let $m=p q$ and $n=r$. An application of Lemma 3 yields that (9) holds under one of the following conditions:

$$
\begin{array}{lll}
\text { (a) } & M \ll X^{\frac{9}{32}}, & N \ll M^{\frac{2}{3}} X^{\frac{1}{10}} ; \\
\text { (b) } & X^{\frac{9}{32}} \ll M \ll X^{\frac{53}{160}}, & N \ll X^{\frac{23}{80}} ; \\
\text { (c) } & X^{\frac{53}{160}} \ll M \ll X^{\frac{13}{32}}, & N \ll M^{-\frac{2}{3}} X^{\frac{61}{120}} ; \\
\text { (d) } & X^{\frac{13}{32}} \ll M \ll X^{\frac{19}{45}}, & N \ll M^{-2} X^{\frac{21}{20}} ; \\
\text { (e) } & X^{\frac{19}{45}} \ll M \ll X^{\frac{897}{1972}}, & N \ll M^{-2} X^{\frac{21}{20}}
\end{array}
$$$$
\text { (d) } \quad X^{\frac{13}{32}} \ll M \ll X^{\frac{19}{45}}, \quad N \ll M^{-2} X^{\frac{21}{20}} \text {; }
$$

Using the same discussion as in Lemma 8 with regions (i) and (ii), we deduce that (9) holds under the condition

$$
X^{\frac{19}{45}} \ll M \ll X^{\frac{897}{1972}}, \quad M^{-2} X^{\frac{21}{20}} \ll N \ll M^{-\frac{1}{5}} X^{\frac{29}{100}} .
$$

In the regions:

$$
\begin{array}{ll}
X^{\frac{9}{85}} \ll P \ll X^{\frac{9}{64}}, & X^{\frac{9}{85}} \ll Q \ll P ; \\
X^{\frac{9}{64}} \ll P \ll X^{\frac{477}{2720}}, & X^{\frac{9}{85}} \ll Q \ll P^{-1} X^{\frac{9}{32}},
\end{array}
$$

condition (a) is satisfied.

In the regions:

$$
\begin{array}{ll}
X^{\frac{9}{64}} \ll P \ll X^{\frac{53}{320}}, & P^{-1} X^{\frac{9}{32}} \ll Q \ll P ; \\
X^{\frac{53}{320}} \ll P \ll X^{\frac{477}{2720}}, & P^{-1} X^{\frac{9}{32}} \ll Q \ll P^{-1} X^{\frac{53}{160}} ; \\
X^{\frac{477}{2720}} \ll P \ll X^{\frac{613}{2720}}, & X^{\frac{9}{85}} \ll Q \ll P^{-1} X^{\frac{53}{160}},
\end{array}
$$

condition (b) is satisfied.

In the regions:

$$
\begin{array}{ll}
X^{\frac{53}{320}} \ll P \ll X^{\frac{13}{64}}, & P^{-1} X^{\frac{53}{160}} \ll Q \ll P ; \\
X^{\frac{13}{64}} \ll P \ll X^{\frac{613}{2720}}, & P^{-1} X^{\frac{53}{160}} \ll Q \ll P^{-1} X^{\frac{13}{32}} ; \\
X^{\frac{613}{2720}} \ll P \ll X^{\frac{817}{2720}}, & X^{\frac{9}{85}} \ll Q \ll P^{-1} X^{\frac{13}{32}},
\end{array}
$$

condition (c) is satisfied. 
In the regions:

$$
\begin{aligned}
& X^{\frac{13}{64}} \ll P \ll X^{\frac{21}{100}}, \quad P^{-1} X^{\frac{13}{32}} \ll Q \ll P ; \\
& X^{\frac{21}{100}} \ll P \ll X^{\frac{13}{60}}, \quad P^{-1} X^{\frac{13}{32}} \ll Q \ll P^{-\frac{2}{3}} X^{\frac{7}{20}} ; \\
& X^{\frac{13}{60}} \ll P \ll X^{\frac{817}{2720}}, \quad P^{-1} X^{\frac{13}{32}} \ll Q \ll P^{-1} X^{\frac{19}{45}} ; \\
& X^{\frac{817}{2720}} \ll P \ll X^{\frac{242}{765}}, \quad X^{\frac{9}{85}} \ll Q \ll P^{-1} X^{\frac{19}{45}},
\end{aligned}
$$

condition (d) is satisfied.

In the regions:

$$
\begin{aligned}
& X^{\frac{13}{60}} \ll P \ll X^{\frac{12613}{49300}}, \quad P^{-1} X^{\frac{19}{45}} \ll Q \ll P^{-\frac{1}{6}} X^{\frac{29}{120}} ; \\
& X^{\frac{12613}{49300}} \ll P \ll X^{\frac{242}{765}}, \quad P^{-1} X^{\frac{19}{45}} \ll Q \ll P^{-1} X^{\frac{897}{1972}} ; \\
& X^{\frac{242}{765}} \ll P \ll X^{\frac{3441}{9860}}, \quad X^{\frac{9}{85}} \ll Q \ll P^{-1} X^{\frac{897}{1972}},
\end{aligned}
$$

conditions (e) and (f) are satisfied.

Putting together the above regions, we get Lemma 14.

LEMma 15. Under the assumption of Lemma 14, assume also that

$$
X^{\frac{9}{85}} \ll R \ll Q
$$

and that $P$ and $Q$ lie in one of the following regions:

$$
\begin{array}{ll}
X^{\frac{297}{800}} \ll P \ll X^{\frac{653}{1700}}, & P^{-1} X^{\frac{49}{100}} \ll Q \ll X^{\frac{19}{160}} \\
X^{\frac{653}{1700}} \ll P \ll X^{\frac{5073}{1320}}, & X^{\frac{9}{85}} \ll Q \ll X^{\frac{19}{160}} ; \\
X^{\frac{5073}{13120}} \ll P \ll X^{\frac{5541}{13940}}, & X^{\frac{9}{85}} \ll Q \ll P^{-\frac{82}{69}} X^{\frac{133}{230}} .
\end{array}
$$

Then (9) holds for $T_{1} \leq T \leq X$.

Proof. Let

$$
m=p q \quad \text { and } \quad n=r .
$$

An application of Lemma 4 yields that (9) holds under the conditions

$$
Q \ll P^{-1} X^{\frac{21}{40}}, \quad X^{\frac{9}{85}} \ll R \ll Q \ll X^{\frac{19}{160}} .
$$

In the regions:

$$
\begin{array}{ll}
X^{\frac{297}{800}} \ll P \ll X^{\frac{653}{1700}}, & P^{-1} X^{\frac{49}{100}} \ll Q \ll X^{\frac{19}{160}} ; \\
X^{\frac{653}{1700}} \ll P \ll X^{\frac{5073}{13120}}, & X^{\frac{9}{85}} \ll Q \ll X^{\frac{19}{160}} ; \\
X^{\frac{5073}{13120}} \ll P \ll X^{\frac{5541}{13940}}, & X^{\frac{9}{85}} \ll Q \ll P^{-\frac{82}{69}} X^{\frac{133}{230}},
\end{array}
$$

the above conditions on $P$ and $Q$ are satisfied.

So the proof of Lemma 15 is complete. 


\section{The remainder term in the sieve method}

Lemma 16. Suppose that $M \ll X^{\frac{21}{40}}, H \ll X^{\frac{19}{160}}$ and that $a(m)=O(1)$, $b(h)=O(1)$. Then for real numbers $x \in(X, 2 X)$, except for a set the measure of which is $O\left(X \log ^{-B} X\right)$, we have

$$
\Sigma=\sum_{\substack{m \sim M \\ h \sim H}} a(m) b(h)\left(\sum_{x<m h l \leq x+\eta x} 1-\frac{\eta x}{m h}\right)=O\left(\eta x \log ^{-B} x\right) .
$$

Proof. If $M H \leq X^{\frac{1}{20}}$, the conclusion is obvious. In the following we suppose

$$
M H>X^{\frac{1}{20}} .
$$

Let $b=1+1 / \log X$ and $M H L=X$. Suppose that $M(s)$ and $H(s)$ are Dirichlet polynomials, $L(s)=\sum_{l \sim L} 1 / l^{s}$ and $F(s)=M(s) H(s) L(s)$. Perron's formula yields

$$
\sum_{\substack{x<m h l \leq x+\eta x \\ m \sim M, h \sim H}} a(m) b(h)=\frac{1}{2 \pi i} \int_{b-i X}^{b+i X} F(s) \frac{(1+\eta)^{s}-1}{s} x^{s} d s+O\left(x^{\varepsilon}\right) .
$$

If $s=b+i t$ and $|t| \leq c_{1} L$, by Theorem 1 on page 442 of [18], we have

$$
\sum_{c_{1} L<l \leq c_{2} L} \frac{1}{l^{s}}=\frac{\left(c_{2} L\right)^{1-s}-\left(c_{1} L\right)^{1-s}}{1-s}+O\left(\frac{1}{L}\right) .
$$

Moreover,

$$
\frac{(1+\eta)^{s}-1}{s} x^{s}=\eta x^{s}+O\left(|s| \eta^{2} x\right), \quad \frac{(1+\eta)^{s}-1}{s} x^{s} \ll \eta x .
$$

Let $T_{1}=\sqrt{L}$. Then

$$
\begin{aligned}
& \frac{1}{2 \pi i} \int_{b-i T_{1}}^{b+i T_{1}} F(s) \frac{(1+\eta)^{s}-1}{s} x^{s} d s \\
& \quad=\frac{\eta}{2 \pi i} \int_{b-i T_{1}}^{b+i T_{1}} M(s) H(s) \frac{\left(c_{2} L\right)^{1-s}-\left(c_{1} L\right)^{1-s}}{1-s} x^{s} d s+O\left(S_{1}\right)+O\left(S_{2}\right),
\end{aligned}
$$

where

$$
\begin{aligned}
& S_{1}=\frac{\eta x}{L} \int_{-T_{1}}^{T_{1}}|M(b+i t) H(b+i t)| d t, \\
& S_{2}=\eta^{2} x \int_{-T_{1}}^{T_{1}}|M(b+i t) H(b+i t)| d t .
\end{aligned}
$$


A trivial estimate yields

$$
S_{1} \ll \frac{\eta x}{\sqrt{L}} \ll \eta x^{1-\varepsilon} \quad \text { and } \quad S_{2} \ll \eta^{2} x \sqrt{L} \ll \eta x^{1-\varepsilon} .
$$

By Perron's formula again,

$$
\begin{aligned}
\frac{\eta}{2 \pi i} \int_{b-i T_{1}}^{b+i T_{1}} M(s) H(s) & \frac{\left(c_{2} L\right)^{1-s}-\left(c_{1} L\right)^{1-s}}{1-s} x^{s} d s \\
& =\eta x \sum_{\substack{m \sim M \\
h \sim H}} \frac{a(m) b(h)}{m h}+O\left(\frac{\eta x^{1+\varepsilon}}{T_{1}}\right)+O\left(\frac{\eta x^{1+\varepsilon}}{M H}\right) \\
& =\eta x \sum_{\substack{m \sim M \\
h \sim H}} \frac{a(m) b(h)}{m h}+O\left(\eta x^{1-\varepsilon}\right) .
\end{aligned}
$$

Now we have

$$
\text { (11) } \begin{aligned}
\Sigma & =\sum_{\substack{x<m h l \leq x+\eta x \\
m \sim M, h \sim H}} a(m) b(h)-\eta x \sum_{\substack{m \sim M \\
h \sim H}} \frac{a(m) b(h)}{m h} \\
& =\frac{1}{2 \pi i} \int_{b-i X}^{b-i T_{1}} F(s) \varrho(s) x^{s} d s+\frac{1}{2 \pi i} \int_{b+i T_{1}}^{b+i X} F(s) \varrho(s) x^{s} d s+O\left(\eta x^{1-\varepsilon}\right),
\end{aligned}
$$

where

$$
\varrho(s)=\frac{(1+\eta)^{s}-1}{s} .
$$

If $s=b+i t$ and $|\operatorname{Im}(s)| \sim T$, then $\varrho(s) \ll \min (\eta, 1 / T)$. We proceed to estimate

$$
\Psi=\left.\left.\int_{X}^{2 X} d x\right|_{b+i T_{1}} ^{b+i X} F(s) \varrho(s) x^{s} d s\right|^{2}
$$

We have

$$
\left.\left.\Psi \ll \log ^{2} x \max _{T_{1} \leq T \leq X} \int_{X}^{2 X} d x\right|_{b+i T} ^{b+2 i T} F(s) \varrho(s) x^{s} d s\right|^{2} .
$$

Here

$$
\int_{X}^{2 X} d x\left|\int_{b+i T}^{b+2 i T} F(s) \varrho(s) x^{s} d s\right|^{2}
$$




$$
\begin{aligned}
& =\int_{X}^{2 X} d x \int_{b+i T}^{b+2 i T} d s_{1} \int_{b+i T}^{b+2 i T} F\left(s_{1}\right) \overline{F\left(s_{2}\right)} \varrho\left(s_{1}\right) \overline{\varrho\left(s_{2}\right)} x^{s_{1}+\bar{s}_{2}} \overline{d s_{2}} \\
& \ll \min ^{2}\left(\eta, \frac{1}{T}\right) \int_{b+i T}^{b+2 i T}\left|d s_{1}\right| \int_{b+i T}^{b+2 i T}\left|F\left(s_{1}\right) F\left(s_{2}\right)\right|\left|\int_{X}^{2 X} x^{s_{1}+\bar{s}_{2}} d x\right|\left|d s_{2}\right| \\
& \ll x^{3} \min ^{2}\left(\eta, \frac{1}{T}\right) \int_{b+i T}^{b+2 i T}\left|d s_{1}\right| \int_{b+i T}^{b+2 i T} \frac{\left|F\left(s_{1}\right)\right|^{2}+\left|F\left(s_{2}\right)\right|^{2}}{\left|1+s_{1}+\bar{s}_{2}\right|}\left|d s_{2}\right| \\
& \ll x^{3} \log x \min ^{2}\left(\eta, \frac{1}{T}\right) \int_{T}^{2 T}|F(b+i t)|^{2} d t .
\end{aligned}
$$

By Lemma 4,

$$
\Psi \ll x^{3} \log ^{3} x \max _{T_{1} \leq T \leq X} \min ^{2}\left(\eta, \frac{1}{T}\right) \int_{T}^{2 T}|F(b+i t)|^{2} d t \ll \eta^{2} x^{3-\varepsilon_{1}} .
$$

Hence, the measure of the set of $x$ satisfying

$$
\left|\int_{b+i T_{1}}^{b+i X} F(s) \varrho(s) x^{s} d s\right| \geq \eta x \log ^{-B} x
$$

is $O\left(X \log ^{-B} X\right)$.

In the same way, we can deal with the integral

$$
\int_{b-i X}^{b-i T_{1}} F(s) \varrho(s) x^{s} d s \text {. }
$$

So the proof of Lemma 16 is complete.

Lemma 17. Suppose that $a(p), b(q), c(r)=O(1)$ and that

$$
X^{\frac{9}{85}} \ll R \ll Q .
$$

Moreover, assume that $P$ and $Q$ lie in one of the following regions:

$$
X^{\frac{9}{85}} \ll P \ll X^{\frac{21}{100}}, \quad X^{\frac{9}{85}} \ll Q \ll P
$$

(iii) $\quad X^{\frac{13}{60}} \ll P \ll X^{\frac{12613}{49300}}, \quad X^{\frac{9}{85}} \ll Q \ll P^{-\frac{1}{6}} X^{\frac{29}{120}}$;

(iv) $\quad X^{\frac{12613}{49300}} \ll P \ll X^{\frac{3441}{9860}}, \quad X^{\frac{9}{85}} \ll Q \ll P^{-1} X^{\frac{897}{1972}}$;

(v) $\quad X^{\frac{297}{800}} \ll P \ll X^{\frac{653}{1700}}, \quad P^{-1} X^{\frac{49}{100}} \ll Q \ll X^{\frac{19}{160}}$;

(vi) $\quad X^{\frac{653}{1700}} \ll P \ll X^{\frac{5073}{13120}}, \quad X^{\frac{9}{85}} \ll Q \ll X^{\frac{19}{160}}$;

(vii) $\quad X^{\frac{5073}{13120}} \ll P \ll X^{\frac{5541}{13940}}, \quad X^{\frac{9}{85}} \ll Q \ll P^{-\frac{82}{69}} X^{\frac{133}{230}}$. 
Let $b=1+1 / \log X, P Q R L=X$ and $T_{1}=\sqrt{L}$. Assume further that for $T_{1} \leq|t| \leq 2 X$

$$
P(b+i t) Q(b+i t) \ll \log ^{-\frac{B}{\varepsilon}} x \quad \text { and } \quad R(b+i t) \ll \log ^{-\frac{B}{\varepsilon}} x .
$$

Then for real numbers $x \in(X, 2 X)$, except for a set the measure of which is $O\left(X \log ^{-B} X\right)$, we have

$$
\sum_{p \sim P} \sum_{q \sim Q} \sum_{r \sim R} a(p) b(q) c(r)\left(\sum_{x<p q r l \leq x+\eta x} 1-\frac{\eta x}{p q r}\right)=O\left(\eta x \log ^{-B} x\right) .
$$

Pro of. Using Lemmas 14, 15 and the discussion in Lemma 16, we get the assertion.

\section{Asymptotic formula}

Lemma 18. Suppose that $X^{\frac{76}{85}} \ll M \ll X^{1-\delta}$ and that $0 \leq a(m)=O(1)$. If $m$ has a prime factor $<X^{\delta}$, then $a(m)=0$. Then for real numbers $x \in(X, 2 X)$, except for a set the measure of which is $O\left(X \log ^{-B} X\right)$, we have

$$
\begin{aligned}
\Sigma & =\sum_{\substack{x<m p \leq x+\eta x \\
m \sim M}} a(m) \\
& =\eta\left(1+O\left(\frac{1}{\log x}\right)\right) \sum_{m \sim M} a(m) \sum_{\frac{x}{m}<p \leq \frac{2 x}{m}} 1+O\left(\eta x \log ^{-B} x\right) .
\end{aligned}
$$

Proof. Let $b=1+1 / \log X$ and $M H=X$. Suppose that $M(s)$ is a Dirichlet polynomial, $H(s)=\sum_{h \sim H} \Lambda(h) / h^{s}$ and $G(s)=M(s) H(s)$. Perron's formula yields

$$
\Sigma_{1}=\sum_{\substack{x<m h \leq x+\eta x \\ m \sim M}} a(m) \Lambda(h)=\frac{1}{2 \pi i} \int_{b-i X}^{b+i X} G(s) \frac{(1+\eta)^{s}-1}{s} x^{s} d s+O\left(x^{\varepsilon}\right) .
$$

Let $T_{0}=\log ^{\frac{B}{\varepsilon}} X$. By (1) and the discussion in Lemma 16,

$$
\begin{aligned}
& \frac{1}{2 \pi i} \int_{b-i T_{0}}^{b+i T_{0}} G(s) \frac{(1+\eta)^{s}-1}{s} x^{s} d s \\
& \quad=\frac{\eta}{2 \pi i} \int_{b-i T_{0}}^{b+i T_{0}} M(s) \frac{\left(c_{2} H\right)^{1-s}-\left(c_{1} H\right)^{1-s}}{1-s} x^{s} d s+O\left(S_{1}\right)+O\left(S_{2}\right),
\end{aligned}
$$

where

$$
S_{1}=\eta x \log ^{-\frac{2 B}{\varepsilon}} x \int_{-T_{0}}^{T_{0}}|M(b+i t)| d t, \quad S_{2}=\eta^{2} x \int_{-T_{0}}^{T_{0}}|M(b+i t)| d t .
$$


A trivial estimate yields

$$
S_{1} \ll \eta x \log ^{-2 B} x \quad \text { and } \quad S_{2} \ll \eta x \log ^{-2 B} x .
$$

By Perron's formula again,

$$
\begin{aligned}
\frac{\eta}{2 \pi i} \int_{b-i T_{0}}^{b+i T_{0}} M(s) \frac{\left(c_{2} H\right)^{1-s}-\left(c_{1} H\right)^{1-s}}{1-s} x^{s} d s \\
=\eta x \sum_{m \sim M} \frac{a(m)}{m}+O\left(\frac{\eta x \log ^{2} x}{T_{0}}\right)+O\left(\frac{\eta x \log ^{2} x}{M}\right) \\
=\eta x \sum_{m \sim M} \frac{a(m)}{m}+O\left(\eta x \log ^{-2 B} x\right) .
\end{aligned}
$$

Hence,

$$
\begin{aligned}
\Sigma_{1}= & \eta x \sum_{m \sim M} \frac{a(m)}{m}+O\left(\eta x \log ^{-2 B} x\right) \\
& +\frac{1}{2 \pi i} \int_{b-i X}^{b-i T_{0}} G(s) \varrho(s) x^{s} d s+\frac{1}{2 \pi i} \int_{b+i T_{0}}^{b+i X} G(s) \varrho(s) x^{s} d s,
\end{aligned}
$$

where

$$
\varrho(s)=\frac{(1+\eta)^{s}-1}{s} .
$$

By Lemma 1 and the discussion in Lemma 16, we have

$$
\frac{1}{2 \pi i} \int_{b+i T_{0}}^{b+i X} G(s) \varrho(s) x^{s} d s=O\left(\eta x \log ^{-2 B} x\right)
$$

and

$$
\frac{1}{2 \pi i} \int_{b-i X}^{b-i T_{0}} G(s) \varrho(s) x^{s} d s=O\left(\eta x \log ^{-2 B} x\right)
$$

for real numbers $x \in(X, 2 X)$, except for a set the measure of which is $O\left(X \log ^{-B} X\right)$. So,

$$
\begin{aligned}
\Sigma_{1} & =\eta x \sum_{m \sim M} \frac{a(m)}{m}+O\left(\eta x \log ^{-2 B} x\right), \\
\Sigma & =\eta x\left(1+O\left(\frac{1}{\log x}\right)\right) \sum_{m \sim M} \frac{a(m)}{m \log (x / m)}+O\left(\eta x \log ^{-B} x\right) \\
& =\eta\left(1+O\left(\frac{1}{\log x}\right)\right) \sum_{m \sim M} a(m) \sum_{\frac{x}{m}<p \leq \frac{2 x}{m}} 1+O\left(\eta x \log ^{-B} x\right) .
\end{aligned}
$$

The proof of Lemma 18 is complete. 
Lemma 19. Suppose that $X^{\frac{9}{85}} \ll H K \ll X^{\frac{76}{85}}, M H K=X$ and that $0 \leq b(h)=O(1), 0 \leq g(k)=O(1)$. If $h$ has a prime factor $<X^{\delta}$, then $b(h)=0$, and similarly for $g(k)$. Suppose that $H(s)$ and $K(s)$ are Dirichlet polynomials, $M(s)=\sum_{m \sim M_{B}} \Lambda(m) / m^{s}$ and $G(s)=M(s) H(s) K(s)$. Let $b=1+1 / \log X$ and $T_{0}=\log ^{\frac{B}{\varepsilon}} X$.

If (7) holds for $T_{0} \leq T \leq X$, then

$$
\begin{aligned}
& \sum_{\substack{x<h k p \leq x+\eta x \\
h \sim H, k \sim K}} b(h) g(k) \\
= & \eta\left(1+O\left(\frac{1}{\log x}\right)\right) \sum_{h \sim H, k \sim K} b(h) g(k) \sum_{\frac{x}{h k}<p \leq \frac{2 x}{h k}} 1+O\left(\eta x \log ^{-B} x\right) .
\end{aligned}
$$

7. Buchstab's function. We define $w(u)$ as the continuous solution of the equations

$$
\begin{cases}w(u)=1 / u, & 1 \leq u \leq 2 \\ (u w(u))^{\prime}=w(u-1), & u>2\end{cases}
$$

$w(u)$ is called Buchstab's function and plays an important role in finding asymptotic formulas in the sieve method. In particular,

$$
\begin{gathered}
w(u)=\frac{1+\log (u-1)}{u}, \quad 2 \leq u \leq 3, \\
w(u)=\frac{1+\log (u-1)}{u}+\frac{1}{u} \int_{2}^{u-1} \frac{\log (t-1)}{t} d t, \quad 3 \leq u \leq 4, \\
w(u)=\frac{1+\log (u-1)}{u}+\frac{1}{u} \int_{2}^{u-1} \frac{\log (t-1)}{t} d t \\
+\frac{1}{u} \int_{3}^{u-1} \frac{d t}{t} \int_{2}^{t-1} \frac{\log (s-1)}{s} d s, \quad 4 \leq u \leq 5 .
\end{gathered}
$$

LEMMA 20. We have the following bounds:

(i) $w(u) \geq 0.5607$ for $u \geq 2.47$,

(ii) $w(u) \leq 0.5644$ for $u \geq 3$,

(iii) $0.5612 \leq w(u) \leq 0.5617$ for $u \geq 4$.

Proof. It is easy to see that $0.5 \leq w(u) \leq 1$ for $1 \leq u \leq 2$. Then we employ induction.

Suppose that $0.5 \leq w(u) \leq 1$ for $1 \leq k \leq u \leq k+1$. If $k+1 \leq u \leq k+2$, 
then (12) yields

$$
u w(u)=(k+1) w(k+1)+\int_{k}^{u-1} w(t) d t .
$$

Hence, $0.5 \leq w(u) \leq 1$ for $k+1 \leq u \leq k+2$. By induction, we obtain $0.5 \leq w(u) \leq 1$ for $u \geq 1$.

If $u>2,(12)$ yields

$$
w^{\prime}(u)=\frac{w(u-1)-w(u)}{u} .
$$

If $2 \leq u \leq 3$, by calculation, we have

$$
\max _{0 \leq k \leq 10^{4}} w\left(2+10^{-4} k\right) \leq 0.56716 .
$$

From (14) and $0.5 \leq w(u) \leq 1$ for $u \geq 1$, it follows that $\left|w^{\prime}(u)\right| \leq \frac{1}{4}$ if $u>2$. Using Lagrange's mean value theorem, we have $w(u) \leq 0.5672$ for $2 \leq u \leq 3$. By induction, we obtain $0.5 \leq w(u) \leq 0.5672$ for $u \geq 2$.

If $3 \leq u \leq 4$, by calculation, we have

$$
\begin{gathered}
\max _{0 \leq k \leq 10^{4}} w\left(3+10^{-4} k\right) \leq 0.56439, \\
\min _{0 \leq k \leq 10^{4}} w\left(3+10^{-4} k\right) \geq 0.56081 .
\end{gathered}
$$

From (14) and $0.5 \leq w(u) \leq 0.5672$ for $u \geq 2$, it follows that $\left|w^{\prime}(u)\right| \leq$ 0.0224 if $u>3$. The above discussion implies that $0.5607 \leq w(u) \leq 0.5644$ for $u \geq 3$. By the same discussion we can also get $0.5607 \leq w(u)$ for $u \geq 2.47$.

If $4 \leq u \leq 5$, by calculation, we have

$$
\max _{0 \leq k \leq 10^{4}} w\left(4+10^{-4} k\right) \leq 0.5616, \quad \min _{0 \leq k \leq 10^{4}} w\left(4+10^{-4} k\right) \geq 0.5613 .
$$

The above discussion and the fact that $\left|w^{\prime}(u)\right| \leq 0.0224$ for $u>3$ imply that $0.5612 \leq w(u) \leq 0.5617$ for $u \geq 4$.

Gathering together the above discussion, we get Lemma 20 .

Lemma 21. Suppose that $\mathcal{E}=\{n: t<n \leq 2 t\}$ and $z \leq t$. Let

$$
P(z)=\prod_{p<z} p \text {. }
$$

Then for sufficiently large $t$ and $z$, we have

$$
S(\mathcal{E}, z)=\sum_{\substack{t<n \leq 2 t \\(n, P(z))=1}} 1=\left(w\left(\frac{\log t}{\log z}\right)+O(\varepsilon)\right) \frac{t}{\log z} .
$$

Proof. See Lemma 5 of [10]. If $(2 t)^{\frac{1}{2}}<z \leq t$, it is the prime number theorem. 
8. Sieve method. We proceed to show that

$$
\pi(x+\eta x)-\pi(x) \geq 0.011 \eta x \log ^{-1} x
$$

for real numbers $x \in(X, 2 X)$, except for a set the measure of which is $O\left(X \log ^{-B} X\right)$.

Let

$$
\begin{gathered}
\mathcal{A}=\{n: x<n \leq x+\eta x\}, \\
P(z)=\prod_{p<z} p, \quad S(\mathcal{A}, z)=\sum_{\substack{a \in \mathcal{A} \\
(a, P(z))=1}} 1 .
\end{gathered}
$$

Then

$$
\pi(x+\eta x)-\pi(x)=S\left(\mathcal{A},(2 X)^{\frac{1}{2}}\right) .
$$

Buchstab's identity yields

$$
\begin{aligned}
S\left(\mathcal{A},(2 X)^{\frac{1}{2}}\right)= & S\left(\mathcal{A}, X^{\frac{9}{85}}\right)-\sum_{X^{\frac{9}{85}}<p \leq(2 X)^{\frac{1}{2}}} S\left(\mathcal{A}_{p}, p\right) \\
= & S\left(\mathcal{A}, X^{\frac{9}{85}}\right)-\sum_{X^{\frac{9}{85}}<p \leq(2 X)^{\frac{1}{2}}} S\left(\mathcal{A}_{p}, X^{\frac{9}{85}}\right) \\
& +\sum_{X^{\frac{9}{85}}<p \leq(2 X)^{\frac{1}{2}}} \sum_{X^{\frac{9}{85}}<q<\min \left(p,(2 X / p)^{\frac{1}{2}}\right)} S\left(\mathcal{A}_{p q}, q\right) .
\end{aligned}
$$

The following lemmas always concern real numbers $x \in(X, 2 X)$, except for a set the measure of which is $O\left(X \log ^{-B} X\right)$.

Let

$$
\mathcal{B}=\{n: x<n \leq 2 x\}
$$

LEMMA 22.

$$
S\left(\mathcal{A}, X^{\frac{9}{85}}\right) \geq 5.300221 \eta x \log ^{-1} x .
$$

Proof. We have

$$
S\left(\mathcal{A}, X^{\frac{9}{85}}\right)=S\left(\mathcal{A}, X^{\delta}\right)-\sum_{X^{\delta}<p \leq X} S_{\frac{9}{85}} S\left(\mathcal{A}_{p}, p\right) .
$$

Let

$$
\widetilde{r}(\mathcal{A}, d)=\left|\mathcal{A}_{d}\right|-\frac{\eta x}{d}, \quad W(z)=\prod_{p<z}\left(1-\frac{1}{p}\right)=(1+O(\varepsilon)) \frac{e^{-\gamma}}{\log z},
$$

where $\gamma$ is Euler's constant. 
Let $z=X^{\delta}$ and $D=X^{\frac{21}{40}}$. Applying Iwaniec's sieve method (see Theorem 1 of [8]), we have

$$
S\left(\mathcal{A}, X^{\delta}\right) \geq \frac{\eta x}{\log z} f\left(\frac{\log D}{\log z}\right)-O\left(\varepsilon \eta x \log ^{-1} x\right)-R^{-},
$$

where

$$
R^{-}=\sum_{m \leq X \frac{21}{40}} a(m) \widetilde{r}(\mathcal{A}, m) .
$$

Lemma 16 yields $R^{-}=O\left(\eta x \log ^{-5} x\right)$. By Theorem 8 on page 181 of [17], we have

$$
f\left(\frac{\log D}{\log z}\right)=e^{-\gamma}+O\left(\varepsilon^{2}\right)
$$

where $\gamma$ is Euler's constant. Thus,

$$
S\left(\mathcal{A}, X^{\delta}\right) \geq \frac{e^{-\gamma}}{\delta} \eta x \log ^{-1} x+O\left(\varepsilon \eta x \log ^{-1} x\right) .
$$

In the same way,

$$
\begin{aligned}
S\left(\mathcal{A}, X^{\delta}\right) & \leq \frac{\eta x}{\log z} F\left(\frac{\log D}{\log z}\right)+O\left(\varepsilon \eta x \log ^{-1} x\right)+\sum_{m \leq X} b(m) \widetilde{r}(\mathcal{A}, m) \\
& \leq \frac{e^{-\gamma}}{\delta} \eta x \log ^{-1} x+O\left(\varepsilon \eta x \log ^{-1} x\right) .
\end{aligned}
$$

So, we have the asymptotic formula

$$
S\left(\mathcal{A}, X^{\delta}\right)=\frac{e^{-\gamma}}{\delta} \eta x \log ^{-1} x+O\left(\varepsilon \eta x \log ^{-1} x\right) .
$$

Now,

$$
\sum_{X^{\delta}<p \leq X^{\frac{9}{85}}} S\left(\mathcal{A}_{p}, p\right)=\sum_{x<p q \leq x+\eta x} 1
$$

where $X^{\delta}<p \leq X^{\frac{9}{85}}$ and the least prime factor of $q$ is greater than $p$.

Using Lemmas 18 and 21 and the prime number theorem, we have

$$
\begin{aligned}
\sum_{X^{\delta}<p \leq X^{\frac{9}{85}}} S\left(\mathcal{A}_{p}, p\right) \\
=\eta \sum_{X^{\delta}<p \leq X^{\frac{9}{85}}} S\left(\mathcal{B}_{p}, p\right)+O\left(\varepsilon \eta x \log ^{-1} x\right) \\
=\eta x \sum_{X^{\delta}<p \leq X^{\frac{9}{85}}} \frac{1}{p \log p} w\left(\frac{\log (x / p)}{\log p}\right)+O\left(\delta \eta x \log ^{-1} x\right)
\end{aligned}
$$




$$
\begin{aligned}
& =\eta x \log ^{-1} x \int_{\delta}^{\frac{9}{85}} \frac{1}{t^{2}} w\left(\frac{1-t}{t}\right) d t+O\left(\delta \eta x \log ^{-1} x\right) \\
& =\eta x \log ^{-1} x \int_{\frac{85}{9}}^{\frac{1}{\delta}} w(u-1) d u+O\left(\delta \eta x \log ^{-1} x\right) \\
& =\eta x \log ^{-1} x\left\{\frac{1}{\delta} w\left(\frac{1}{\delta}\right)-\frac{85}{9} w\left(\frac{85}{9}\right)\right\}+O\left(\delta \eta x \log ^{-1} x\right) \\
& =\frac{e^{-\gamma}}{\delta} \eta x \log ^{-1} x-\frac{85}{9} w\left(\frac{85}{9}\right) \eta x \log ^{-1} x+O\left(\delta \eta x \log ^{-1} x\right),
\end{aligned}
$$

since $w(1 / \delta)=e^{-\gamma}+O\left(\varepsilon^{2}\right)$ (see Lemma 12 on page 179 of [17]). Hence,

$$
S\left(\mathcal{A}, X^{\frac{9}{85}}\right)=\frac{85}{9} w\left(\frac{85}{9}\right) \eta x \log ^{-1} x+O\left(\delta \eta x \log ^{-1} x\right) .
$$

By Lemma 20, we get $S\left(\mathcal{A}, X^{\frac{9}{85}}\right) \geq \frac{85}{9} \cdot 0.5612 \eta x \log ^{-1} x+O\left(\delta \eta x \log ^{-1} x\right) \geq 5.300221 \eta x \log ^{-1} x$.

So the proof of Lemma 22 is complete.

LEMMA 23.

$$
\sum_{X^{\frac{9}{85}<p \leq(2 X)^{\frac{1}{2}}}} S\left(\mathcal{A}_{p}, X^{\frac{9}{85}}\right) \leq 8.234757 \eta x \log ^{-1} x .
$$

Proof. Buchstab's identity yields

$$
\begin{aligned}
& \sum_{X^{\frac{9}{85}<p \leq(2 X)^{\frac{1}{2}}}} S\left(\mathcal{A}_{p}, X^{\frac{9}{85}}\right) \\
& \quad=\sum_{X^{\frac{9}{85}}<p \leq(2 X)^{\frac{1}{2}}} S\left(\mathcal{A}_{p}, X^{\delta}\right)-\sum_{X^{\frac{9}{85}<p \leq(2 X)^{\frac{1}{2}}}} \sum_{X^{\delta}<q<X^{\frac{9}{85}}} S\left(\mathcal{A}_{p q}, q\right) .
\end{aligned}
$$

Using Lemma 16, in the same way as in Lemma 22, we have

$$
\begin{aligned}
\sum_{X^{\frac{9}{85}<p \leq(2 X)^{\frac{1}{2}}}} S\left(\mathcal{A}_{p}, X^{\delta}\right) \\
=\sum_{X^{\frac{9}{85}<p \leq(2 X)^{\frac{1}{2}}}} \frac{e^{-\gamma}}{\delta} \cdot \frac{1}{p} \cdot \eta x \log ^{-1} x+O\left(\varepsilon \eta x \log ^{-1} x\right) \\
=\frac{e^{-\gamma}}{\delta} \cdot \eta x \log ^{-1} x \int_{\frac{9}{85}}^{\frac{1}{2}} \frac{d t}{t}+O\left(\varepsilon \eta x \log ^{-1} x\right) .
\end{aligned}
$$


Using Lemmas 18 and 21, in the same way as in Lemma 22, we have

$$
\begin{aligned}
& \sum_{X \frac{9}{85}<p \leq(2 X)^{\frac{1}{2}}} \sum_{X^{\delta}<q<X \frac{9}{85}} S\left(\mathcal{A}_{p q}, q\right) \\
& =\eta \sum_{X^{\frac{9}{85}}<p \leq(2 X)^{\frac{1}{2}}} \sum_{X^{\delta}<q<X} S\left(\mathcal{B}_{p q}, q\right)+O\left(\varepsilon \eta x \log ^{-1} x\right) \\
& =\eta x \sum_{X^{\frac{9}{85}}<p \leq(2 X)^{\frac{1}{2}}} \sum_{X^{\delta}<q<X^{\frac{9}{85}}} \frac{1}{p q \log q} w\left(\frac{\log (x /(p q))}{\log q}\right) \\
& +O\left(\delta \eta x \log ^{-1} x\right) \\
& =\eta x \log ^{-1} x \int_{\frac{9}{85}}^{\frac{1}{2}} \frac{d t}{t} \int_{\delta}^{\frac{9}{85}} \frac{1}{u^{2}} w\left(\frac{1-t-u}{u}\right) d u+O\left(\delta \eta x \log ^{-1} x\right) \\
& =\eta x \log ^{-1} x \int_{\frac{9}{85}}^{\frac{1}{2}} \frac{d t}{t(1-t)} \int_{\frac{85}{9}(1-t)}^{\frac{1}{\delta}(1-t)} w(r-1) d r+O\left(\delta \eta x \log ^{-1} x\right) \\
& =\frac{1}{\delta} \cdot \eta x \log ^{-1} x \int_{\frac{9}{85}}^{\frac{1}{2}} \frac{1}{t} w\left(\frac{1}{\delta}(1-t)\right) d t \\
& -\frac{85}{9} \cdot \eta x \log ^{-1} x \int_{\frac{9}{85}}^{\frac{1}{2}} \frac{1}{t} w\left(\frac{85}{9}(1-t)\right) d t+O\left(\delta \eta x \log ^{-1} x\right) \\
& =\frac{e^{-\gamma}}{\delta} \cdot \eta x \log ^{-1} x \int_{\frac{9}{85}}^{\frac{1}{2}} \frac{d t}{t}-\frac{85}{9} \cdot \eta x \log ^{-1} x \int_{\frac{9}{85}}^{\frac{1}{2}} \frac{1}{t} w\left(\frac{85}{9}(1-t)\right) d t \\
& +O\left(\delta \eta x \log ^{-1} x\right) .
\end{aligned}
$$

Gathering together the above discussion and applying Lemma 20, we have

$$
\begin{aligned}
\sum_{X^{\frac{9}{85}<p \leq(2 X)^{\frac{1}{2}}}} S\left(\mathcal{A}_{p}, X^{\frac{9}{85}}\right) \\
=\frac{85}{9} \cdot \eta x \log ^{-1} x \int_{\frac{9}{85}}^{\frac{1}{2}} \frac{1}{t} w\left(\frac{85}{9}(1-t)\right) d t+O\left(\delta \eta x \log ^{-1} x\right)
\end{aligned}
$$




$$
\begin{aligned}
& \leq 0.5617 \cdot \frac{85}{9} \cdot \log \left(\frac{85}{18}\right) \eta x \log ^{-1} x+O\left(\delta \eta x \log ^{-1} x\right) \\
& \leq 8.234757 \eta x \log ^{-1} x .
\end{aligned}
$$

So the proof of Lemma 23 is complete.

We now set

$$
\begin{aligned}
\Omega & \sum_{X \frac{9}{85}<p \leq(2 X)^{\frac{1}{2}}} \sum_{X^{\frac{9}{85}}<q<\min \left(p,\left(\frac{2 X}{p}\right)^{\frac{1}{2}}\right)} S\left(\mathcal{A}_{p q}, q\right) \\
& \geq \sum_{i=1}^{94} \sum_{(p, q) \in D_{i}} S\left(\mathcal{A}_{p q}, q\right)=\sum_{i=1}^{94} \Omega_{i},
\end{aligned}
$$

where

$$
\begin{aligned}
& D_{1}=\left\{(p, q): X^{\frac{9}{85}}<p \leq X^{\frac{21}{100}}, X^{\frac{9}{85}}<q<p\right\}, \\
& D_{2}=\left\{(p, q): X^{\frac{21}{100}}<p \leq X^{\frac{13}{60}}, X^{\frac{9}{85}}<q<p^{-\frac{2}{3}} X^{\frac{7}{20}}\right\} \text {, } \\
& D_{3}=\left\{(p, q): X^{\frac{13}{60}}<p \leq X^{\frac{133}{580}}, X^{\frac{9}{85}}<q<p^{-\frac{1}{6}} X^{\frac{29}{120}}\right\} \text {, } \\
& D_{4}=\left\{(p, q): X^{\frac{133}{580}}<p \leq X^{\frac{19}{80}}, X^{\frac{9}{85}}<q<p^{-\frac{1}{6}} X^{\frac{29}{120}}\right\} \text {, } \\
& D_{5}=\left\{(p, q): X^{\frac{133}{580}}<p \leq X^{\frac{19}{80}}, p^{-1} X^{\frac{133}{290}}<q<p\right\} \text {, } \\
& D_{6}=\left\{(p, q): X^{\frac{19}{80}}<p \leq X^{\frac{53}{220}}, X^{\frac{9}{85}}<q<p^{-\frac{1}{6}} X^{\frac{29}{120}}\right\} \text {, } \\
& D_{7}=\left\{(p, q): X^{\frac{19}{80}}<p \leq X^{\frac{53}{220}}, p^{-1} X^{\frac{133}{290}}<q<p^{-1} X^{\frac{19}{40}}\right\} \text {, } \\
& D_{8}=\left\{(p, q): X^{\frac{53}{220}}<p \leq X^{\frac{12613}{49300}}, X^{\frac{9}{85}}<q<p^{-\frac{1}{6}} X^{\frac{29}{120}}\right\} \text {, } \\
& D_{9}=\left\{(p, q): X^{\frac{53}{220}}<p \leq X^{\frac{12613}{49300}}, p^{-1} X^{\frac{133}{290}}<q<p^{-1} X^{\frac{53}{110}}\right\} \text {, } \\
& D_{10}=\left\{(p, q): X^{\frac{12613}{49300}}<p \leq X^{\frac{151}{580}}, X^{\frac{9}{85}}<q<p^{-1} X^{\frac{897}{1972}}\right\} \text {, } \\
& D_{11}=\left\{(p, q): X^{\frac{12613}{49300}}<p \leq X^{\frac{151}{580}}, p^{-1} X^{\frac{133}{290}}<q<p^{-1} X^{\frac{53}{110}}\right\} \text {, } \\
& D_{12}=\left\{(p, q): X^{\frac{151}{580}}<p \leq X^{\frac{19}{70}}, X^{\frac{9}{85}}<q<p^{-1} X^{\frac{897}{1972}}\right\} \text {, } \\
& D_{13}=\left\{(p, q): X^{\frac{151}{580}}<p \leq X^{\frac{19}{70}}, p^{-1} X^{\frac{897}{1972}}<q<p^{-1} X^{\frac{53}{110}}\right\} \text {, } \\
& D_{14}=\left\{(p, q): X^{\frac{19}{70}}<p \leq X^{\frac{3}{10}}, X^{\frac{9}{85}}<q<p^{-1} X^{\frac{897}{1972}}\right\} \text {, } \\
& D_{15}=\left\{(p, q): X^{\frac{19}{70}}<p \leq X^{\frac{3}{10}}, p^{-1} X^{\frac{897}{1972}}<q<p^{-1} X^{\frac{53}{110}}\right\}, \\
& D_{16}=\left\{(p, q): X^{\frac{19}{70}}<p \leq X^{\frac{3}{10}}, p^{-\frac{6}{5}} X^{\frac{57}{100}}<q<p^{-\frac{1}{8}} X^{\frac{23}{80}}\right\} \text {, } \\
& D_{17}=\left\{(p, q): X^{\frac{3}{10}}<p \leq X^{\frac{17}{55}}, X^{\frac{9}{85}}<q<p^{-1} X^{\frac{897}{1972}}\right\} \text {, }
\end{aligned}
$$




$$
\begin{aligned}
& D_{18}=\left\{(p, q): X^{\frac{3}{10}}<p \leq X^{\frac{17}{55}}, p^{-1} X^{\frac{897}{1972}}<q<p^{-1} X^{\frac{53}{110}}\right\}, \\
& D_{19}=\left\{(p, q): X^{\frac{3}{10}}<p \leq X^{\frac{17}{55}}, p^{-\frac{12}{11}} X^{\frac{57}{110}}<q<p^{-\frac{1}{8}} X^{\frac{23}{80}}\right\} \text {, } \\
& D_{20}=\left\{(p, q): X^{\frac{17}{55}}<p \leq X^{\frac{19}{60}}, X^{\frac{9}{85}}<q<p^{-1} X^{\frac{897}{1972}}\right\} \text {, } \\
& D_{21}=\left\{(p, q): X^{\frac{17}{55}}<p \leq X^{\frac{19}{60}}, p^{-1} X^{\frac{897}{1972}}<q<p^{-\frac{58}{67}} X^{\frac{59}{134}}\right\} \text {, } \\
& D_{22}=\left\{(p, q): X^{\frac{17}{55}}<p \leq X^{\frac{19}{60}}, p^{-\frac{12}{11}} X^{\frac{57}{110}}<q<p^{-\frac{1}{8}} X^{\frac{23}{80}}\right\}, \\
& D_{23}=\left\{(p, q): X^{\frac{19}{60}}<p \leq X^{\frac{247}{770}}, X^{\frac{9}{85}}<q<p^{-1} X^{\frac{897}{1972}}\right\}, \\
& D_{24}=\left\{(p, q): X^{\frac{19}{60}}<p \leq X^{\frac{247}{770}}, p^{-1} X^{\frac{897}{1972}}<q<p^{-\frac{58}{67}} X^{\frac{59}{134}}\right\} \text {, } \\
& D_{25}=\left\{(p, q): X^{\frac{19}{60}}<p \leq X^{\frac{247}{770}}, X^{\frac{19}{110}}<q<p^{-\frac{1}{8}} X^{\frac{23}{80}}\right\} \text {, } \\
& D_{26}=\left\{(p, q): X^{\frac{247}{770}}<p \leq X^{\frac{359}{1100}}, X^{\frac{9}{85}}<q<p^{-1} X^{\frac{897}{1972}}\right\} \text {, } \\
& D_{27}=\left\{(p, q): X^{\frac{247}{770}}<p \leq X^{\frac{359}{1100}}, p^{-1} X^{\frac{897}{1972}}<q<p^{-\frac{58}{67}} X^{\frac{59}{134}}\right\} \text {, } \\
& D_{28}=\left\{(p, q): X^{\frac{247}{770}}<p \leq X^{\frac{359}{1100}}, X^{\frac{19}{110}}<q<p^{-1} X^{\frac{25}{44}}\right\}, \\
& D_{29}=\left\{(p, q): X^{\frac{359}{1100}}<p \leq X^{\frac{481}{1450}}, X^{\frac{9}{85}}<q<p^{-1} X^{\frac{897}{1972}}\right\}, \\
& D_{30}=\left\{(p, q): X^{\frac{359}{1100}}<p \leq X^{\frac{481}{1450}}, p^{-1} X^{\frac{897}{1972}}<q<p^{-\frac{58}{67}} X^{\frac{59}{134}}\right\} \text {, } \\
& D_{31}=\left\{(p, q): X^{\frac{359}{1100}}<p \leq X^{\frac{481}{1450}}, X^{\frac{19}{110}}<q<p^{-6} X^{\frac{11}{5}}\right\}, \\
& D_{32}=\left\{(p, q): X^{\frac{481}{1450}}<p \leq X^{\frac{443}{1305}}, X^{\frac{9}{85}}<q<p^{-1} X^{\frac{897}{1972}}\right\}, \\
& D_{33}=\left\{(p, q): X^{\frac{481}{1450}}<p \leq X^{\frac{443}{1305}}, p^{-1} X^{\frac{897}{1972}}<q<p^{-\frac{58}{67}} X^{\frac{59}{134}}\right\} \text {, } \\
& D_{34}=\left\{(p, q): X^{\frac{481}{1450}}<p \leq X^{\frac{443}{1305}}, X^{\frac{19}{110}}<q<p^{-1} X^{\frac{157}{290}}\right\}, \\
& D_{35}=\left\{(p, q): X^{\frac{443}{1305}}<p \leq X^{\frac{19}{55}}, X^{\frac{9}{85}}<q<p^{-1} X^{\frac{897}{1972}}\right\}, \\
& D_{36}=\left\{(p, q): X^{\frac{443}{1305}}<p \leq X^{\frac{19}{55}}, p^{-1} X^{\frac{897}{1972}}<q<p^{-\frac{58}{67}} X^{\frac{59}{134}}\right\} \text {, } \\
& D_{37}=\left\{(p, q): X^{\frac{443}{1305}}<p \leq X^{\frac{19}{55}}, X^{\frac{19}{110}}<q<p^{-\frac{2}{11}} X^{\frac{29}{110}}\right\} \text {, } \\
& D_{38}=\left\{(p, q): X^{\frac{19}{55}}<p \leq X^{\frac{3441}{9860}}, X^{\frac{9}{85}}<q<p^{-1} X^{\frac{897}{1972}}\right\} \text {, } \\
& D_{39}=\left\{(p, q): X^{\frac{19}{55}}<p \leq X^{\frac{3441}{9860}}, p^{-1} X^{\frac{897}{1972}}<q<p^{-\frac{58}{67}} X^{\frac{59}{134}}\right\} \text {, } \\
& D_{40}=\left\{(p, q): X^{\frac{19}{55}}<p \leq X^{\frac{3441}{9860}}, p^{-\frac{58}{49}} X^{\frac{57}{98}}<q<p^{-\frac{2}{11}} X^{\frac{29}{110}}\right\} \text {, } \\
& D_{41}=\left\{(p, q): X^{\frac{3441}{9860}}<p \leq X^{\frac{1843}{5280}}, X^{\frac{9}{85}}<q<p^{-\frac{58}{67}} X^{\frac{59}{134}}\right\} \text {, } \\
& D_{42}=\left\{(p, q): X^{\frac{3441}{9860}}<p \leq X^{\frac{1843}{5280}}, p^{-\frac{58}{49}} X^{\frac{57}{98}}<q<p^{-\frac{2}{11}} X^{\frac{29}{110}}\right\} \text {, }
\end{aligned}
$$




$$
\begin{aligned}
& D_{43}=\left\{(p, q): X^{\frac{1843}{5280}}<p \leq X^{\frac{37}{100}}, X^{\frac{9}{85}}<q<p^{-\frac{58}{67}} X^{\frac{59}{134}}\right\}, \\
& D_{44}=\left\{(p, q): X^{\frac{1843}{5800}}<p \leq X^{\frac{37}{100}}, p^{-\frac{70}{59}} X^{\frac{171}{295}}<q<p^{-\frac{58}{49}} X^{\frac{57}{98}}\right\} \text {, } \\
& D_{45}=\left\{(p, q): X^{\frac{1843}{5280}}<p \leq X^{\frac{37}{100}}, p^{-\frac{58}{49}} X^{\frac{57}{98}}<q<p^{-\frac{2}{11}} X^{\frac{29}{110}}\right\} \text {, } \\
& D_{46}=\left\{(p, q): X^{\frac{37}{100}}<p \leq X^{\frac{297}{800}}, X^{\frac{9}{85}}<q<p^{-1} X^{\frac{49}{100}}\right\}, \\
& D_{47}=\left\{(p, q): X^{\frac{37}{100}}<p \leq X^{\frac{297}{800}}, p^{-\frac{82}{69}} X^{\frac{133}{230}}<q<p^{-\frac{58}{49}} X^{\frac{57}{98}}\right\} \text {, } \\
& D_{48}=\left\{(p, q): X^{\frac{37}{100}}<p \leq X^{\frac{297}{800}}, p^{-\frac{58}{49}} X^{\frac{57}{98}}<q<p^{-\frac{2}{11}} X^{\frac{29}{110}}\right\} \text {, } \\
& D_{49}=\left\{(p, q): X^{\frac{297}{800}}<p \leq X^{\frac{2143}{5620}}, X^{\frac{9}{85}}<q<p^{-1} X^{\frac{49}{100}}\right\} \text {, } \\
& D_{50}=\left\{(p, q): X^{\frac{297}{800}}<p \leq X^{\frac{2143}{5620}}, p^{-1} X^{\frac{49}{100}}<q<X^{\frac{19}{160}}\right\}, \\
& D_{51}=\left\{(p, q): X^{\frac{297}{800}}<p \leq X^{\frac{2143}{5620}}, p^{-\frac{82}{69}} X^{\frac{133}{230}}<q<p^{-\frac{58}{49}} X^{\frac{57}{98}}\right\} \text {, } \\
& D_{52}=\left\{(p, q): X^{\frac{297}{800}}<p \leq X^{\frac{2143}{5620}}, p^{-\frac{58}{49}} X^{\frac{57}{98}}<q<p^{-\frac{2}{11}} X^{\frac{29}{110}}\right\} \text {, } \\
& D_{53}=\left\{(p, q): X^{\frac{2143}{5620}}<p \leq X^{\frac{653}{1700}}, X^{\frac{9}{85}}<q<p^{-1} X^{\frac{49}{100}}\right\}, \\
& D_{54}=\left\{(p, q): X^{\frac{2143}{5620}}<p \leq X^{\frac{653}{1700}}, p^{-1} X^{\frac{49}{100}}<q<X^{\frac{19}{160}}\right\}, \\
& D_{55}=\left\{(p, q): X^{\frac{2143}{5620}}<p \leq X^{\frac{653}{1700}}, p^{-\frac{82}{69}} X^{\frac{133}{230}}<q<p^{-\frac{58}{49}} X^{\frac{57}{98}}\right\} \text {, } \\
& D_{56}=\left\{(p, q): X^{\frac{2143}{5620}}<p \leq X^{\frac{653}{1700}}, p^{-\frac{58}{49}} X^{\frac{57}{98}}<q<p^{-\frac{29}{19}} X^{\frac{59}{76}}\right\} \text {, } \\
& D_{57}=\left\{(p, q): X^{\frac{653}{1700}}<p \leq X^{\frac{5073}{13120}}, X^{\frac{9}{85}}<q<X^{\frac{19}{160}}\right\}, \\
& D_{58}=\left\{(p, q): X^{\frac{653}{1700}}<p \leq X^{\frac{5073}{13120}}, p^{-\frac{82}{69}} X^{\frac{133}{230}}<q<p^{-\frac{58}{49}} X^{\frac{57}{98}}\right\}, \\
& D_{59}=\left\{(p, q): X^{\frac{653}{1700}}<p \leq X^{\frac{5073}{13120}}, p^{-\frac{58}{49}} X^{\frac{57}{98}}<q<p^{-\frac{29}{19}} X^{\frac{59}{76}}\right\}, \\
& D_{60}=\left\{(p, q): X^{\frac{5073}{13120}}<p \leq X^{\frac{5541}{13940}}, X^{\frac{9}{85}}<q<p^{-\frac{82}{69}} X^{\frac{133}{230}}\right\}, \\
& D_{61}=\left\{(p, q): X^{\frac{5073}{13120}}<p \leq X^{\frac{5541}{13940}}, p^{-\frac{82}{69}} X^{\frac{133}{230}}<q<p^{-\frac{58}{49}} X^{\frac{57}{98}}\right\} \text {, } \\
& D_{62}=\left\{(p, q): X^{\frac{5073}{13120}}<p \leq X^{\frac{5541}{13940}}, p^{-\frac{58}{49}} X^{\frac{57}{98}}<q<p^{-\frac{29}{19}} X^{\frac{59}{76}}\right\}, \\
& D_{63}=\left\{(p, q): X^{\frac{5541}{13940}}<p \leq X^{\frac{3963}{9860}}, X^{\frac{9}{85}}<q<p^{-\frac{58}{49}} X^{\frac{57}{98}}\right\} \text {, } \\
& D_{64}=\left\{(p, q): X^{\frac{5541}{13940}}<p \leq X^{\frac{3963}{9860}}, p^{-\frac{58}{49}} X^{\frac{57}{98}}<q<p^{-\frac{29}{19}} X^{\frac{59}{76}}\right\} \text {, } \\
& D_{65}=\left\{(p, q): X^{\frac{3963}{9860}}<p \leq X^{\frac{1063}{2640}}, X^{\frac{9}{85}}<q<p^{-\frac{29}{19}} X^{\frac{59}{76}}\right\} \text {, } \\
& D_{66}=\left\{(p, q): X^{\frac{1063}{2640}}<p \leq X^{\frac{19}{45}}, X^{\frac{9}{85}}<q<p^{-\frac{29}{19}} X^{\frac{59}{76}}\right\} \text {, } \\
& D_{67}=\left\{(p, q): X^{\frac{1063}{2640}}<p \leq X^{\frac{19}{45}}, p^{-\frac{29}{19}} X^{\frac{59}{76}}<q<p^{-\frac{35}{23}} X^{\frac{179}{230}}\right\} \text {, }
\end{aligned}
$$




$$
\begin{aligned}
& D_{68}=\left\{(p, q): X^{\frac{19}{45}}<p \leq X^{\frac{19}{44}}, X^{\frac{9}{85}}<q<p^{-\frac{29}{19}} X^{\frac{59}{76}}\right\}, \\
& D_{69}=\left\{(p, q): X^{\frac{19}{45}}<p \leq X^{\frac{19}{44}}, p^{-\frac{29}{19}} X^{\frac{59}{76}}<q<p^{-\frac{41}{27}} X^{\frac{421}{540}}\right\} \text {, } \\
& D_{70}=\left\{(p, q): X^{\frac{19}{45}}<p \leq X^{\frac{19}{44}}, p^{-\frac{29}{10}} X^{\frac{57}{40}}<q<p^{-\frac{1}{5}} X^{\frac{29}{100}}\right\}, \\
& D_{71}=\left\{(p, q): X^{\frac{19}{44}}<p \leq X^{\frac{4331}{9860}}, X^{\frac{9}{85}}<q<p^{-\frac{29}{19}} X^{\frac{59}{76}}\right\} \text {, } \\
& D_{72}=\left\{(p, q): X^{\frac{19}{44}}<p \leq X^{\frac{4331}{9860}}, p^{-\frac{29}{19}} X^{\frac{59}{76}}<q<p^{-\frac{41}{27}} X^{\frac{421}{540}}\right\} \text {, } \\
& D_{73}=\left\{(p, q): X^{\frac{19}{44}}<p \leq X^{\frac{4331}{9860}}, p^{-\frac{29}{10}} X^{\frac{57}{40}}<q<p^{-\frac{1}{5}} X^{\frac{29}{100}}\right\} \text {, } \\
& D_{74}=\left\{(p, q): X^{\frac{19}{44}}<p \leq X^{\frac{4331}{9860}}, p^{-\frac{6}{5}} X^{\frac{19}{25}}<q<p^{\frac{1}{7}} X^{\frac{13}{70}}\right\} \text {, } \\
& D_{75}=\left\{(p, q): X^{\frac{4331}{9860}}<p \leq X^{\frac{2691}{5950}}, p^{-\frac{35}{12}} X^{\frac{57}{40}}<q<p^{-\frac{29}{10}} X^{\frac{57}{40}}\right\} \text {, } \\
& D_{76}=\left\{(p, q): X^{\frac{4331}{9860}}<p \leq X^{\frac{2691}{5950}}, p^{-\frac{29}{10}} X^{\frac{57}{40}}<q<p^{-\frac{1}{5}} X^{\frac{29}{100}}\right\} \text {, } \\
& D_{77}=\left\{(p, q): X^{\frac{4331}{9860}}<p \leq X^{\frac{2691}{5950}}, p^{-\frac{6}{5}} X^{\frac{19}{25}}<q<p^{\frac{1}{7}} X^{\frac{13}{70}}\right\} \text {, } \\
& D_{78}=\left\{(p, q): X^{\frac{2691}{5950}}<p \leq X^{\frac{897}{1972}}, X^{\frac{9}{85}}<q<p^{-\frac{29}{10}} X^{\frac{57}{40}}\right\} \text {, } \\
& D_{79}=\left\{(p, q): X^{\frac{2691}{5950}}<p \leq X^{\frac{897}{1972}}, p^{-\frac{29}{10}} X^{\frac{57}{40}}<q<p^{-\frac{1}{5}} X^{\frac{29}{100}}\right\} \text {, } \\
& D_{80}=\left\{(p, q): X^{\frac{2691}{5950}}<p \leq X^{\frac{897}{1972}}, p^{-\frac{6}{5}} X^{\frac{19}{25}}<q<p^{\frac{1}{7}} X^{\frac{13}{70}}\right\}, \\
& D_{81}=\left\{(p, q): X^{\frac{897}{1972}}<p \leq X^{\frac{133}{290}}, X^{\frac{9}{85}}<q<p^{-\frac{1}{5}} X^{\frac{29}{100}}\right\} \text {, } \\
& D_{82}=\left\{(p, q): X^{\frac{897}{1972}}<p \leq X^{\frac{133}{290}}, p^{-\frac{6}{5}} X^{\frac{19}{25}}<q<p^{\frac{1}{7}} X^{\frac{13}{70}}\right\}, \\
& D_{83}=\left\{(p, q): X^{\frac{133}{290}}<p \leq X^{\frac{19}{40}}, X^{\frac{9}{85}}<q<p^{\frac{1}{7}} X^{\frac{13}{70}}\right\} \text {, } \\
& D_{84}=\left\{(p, q): X^{\frac{19}{40}}<p \leq X^{\frac{53}{110}}, X^{\frac{9}{85}}<q<p^{-1} X^{\frac{51}{70}}\right\} \text {, } \\
& D_{85}=\left\{(p, q): X^{\frac{53}{110}}<p \leq X^{\frac{339}{700}}, X^{\frac{9}{85}}<q<p^{-\frac{58}{9}} X^{\frac{59}{18}}\right\} \text {, } \\
& D_{86}=\left\{(p, q): X^{\frac{53}{110}}<p \leq X^{\frac{339}{700}}, X^{\frac{19}{110}}<q<p^{-1} X^{\frac{51}{70}}\right\} \text {, } \\
& D_{87}=\left\{(p, q): X^{\frac{339}{700}}<p \leq X^{\frac{233}{480}}, X^{\frac{9}{85}}<q<p^{-\frac{58}{9}} X^{\frac{59}{18}}\right\} \text {, } \\
& D_{88}=\left\{(p, q): X^{\frac{339}{700}}<p \leq X^{\frac{233}{480}}, X^{\frac{19}{110}}<q<p^{-6} X^{\frac{63}{20}}\right\}, \\
& D_{89}=\left\{(p, q): X^{\frac{233}{480}}<p \leq X^{\frac{49}{100}}, X^{\frac{9}{85}}<q<p^{-\frac{58}{9}} X^{\frac{59}{18}}\right\} \text {, } \\
& D_{90}=\left\{(p, q): X^{\frac{233}{480}}<p \leq X^{\frac{49}{100}}, p^{-\frac{58}{9}} X^{\frac{59}{18}}<q<p^{-\frac{70}{11}} X^{\frac{179}{55}}\right\} \text {, } \\
& D_{91}=\left\{(p, q): X^{\frac{233}{480}}<p \leq X^{\frac{49}{100}}, X^{\frac{19}{110}}<q<p^{-6} X^{\frac{63}{20}}\right\} \text {, } \\
& D_{92}=\left\{(p, q): X^{\frac{49}{100}}<p \leq X^{\frac{2944}{5950}}, X^{\frac{9}{85}}<q<p^{-\frac{70}{11}} X^{\frac{179}{55}}\right\} \text {, }
\end{aligned}
$$




$$
\begin{aligned}
& D_{93}=\left\{(p, q): X^{\frac{49}{100}}<p \leq X^{\frac{2944}{5950}}, X^{\frac{19}{110}}<q<p^{-1} X^{\frac{7}{10}}\right\}, \\
& D_{94}=\left\{(p, q): X^{\frac{2944}{5950}}<p \leq X^{\frac{1}{2}}, X^{\frac{19}{110}}<q<p^{-1} X^{\frac{7}{10}}\right\} .
\end{aligned}
$$

\section{Bounds of the sieve functions}

LEMMA 24.

$$
\begin{aligned}
\Omega_{16} & +\Omega_{19}+\Omega_{22}+\Omega_{25}+\Omega_{28}+\Omega_{31}+\Omega_{34}+\Omega_{37}+\Omega_{40}+\Omega_{42}+\Omega_{45} \\
& +\Omega_{48}+\Omega_{52}+\Omega_{56}+\Omega_{59}+\Omega_{62}+\Omega_{64}+\Omega_{65}+\Omega_{66}+\Omega_{68}+\Omega_{71} \\
\geq & 0.394443 \eta x \log ^{-1} x .
\end{aligned}
$$

Proof. We have

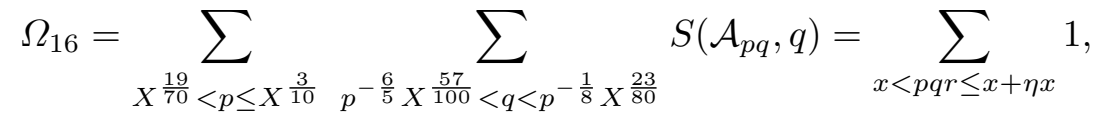

where $X^{\frac{19}{70}}<p \leq X^{\frac{3}{10}}, p^{-\frac{6}{5}} X^{\frac{57}{100}}<q<p^{-\frac{1}{8}} X^{\frac{23}{80}}$ and the least prime factor of $r$ is greater than $q$.

Let $h=q$ and $k=r$. By Lemma 6 with region (i), (7) holds. Then Lemmas 19 and 21 yield

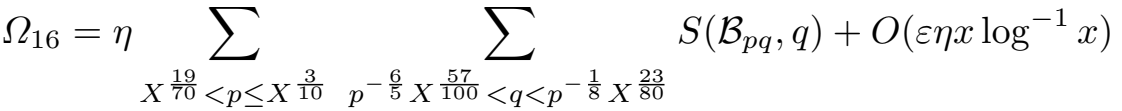

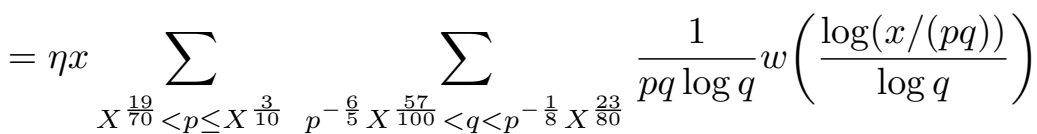

$$
\begin{aligned}
& +O\left(\varepsilon \eta x \log ^{-1} x\right) \\
& =\eta x \log ^{-1} x \int_{\frac{19}{70}}^{\frac{3}{10}} \frac{d t}{t} \int_{\frac{57}{100}-\frac{6}{5} t}^{\frac{23}{80}-\frac{t}{8}} \frac{1}{u^{2}} w\left(\frac{1-t-u}{u}\right) d u+O\left(\varepsilon \eta x \log ^{-1} x\right) \\
& =\eta x \log ^{-1} x\left(\int_{\frac{19}{70}}^{\frac{71}{260}} \frac{d t}{t} \int_{\frac{57}{100}-\frac{6}{5} t}^{\frac{23}{80}-\frac{t}{8}} \frac{d u}{u(1-t-u)}\right. \\
& +\int_{\frac{71}{260}}^{\frac{3}{10}} \frac{d t}{t} \int_{\frac{57}{100}-\frac{6}{5} t}^{\frac{1}{3}(1-t)} \frac{1}{u(1-t-u)}\left(1+\log \left(\frac{1-t}{u}-2\right)\right) d u \\
& \left.+\int_{\frac{71}{260}}^{\frac{3}{10}} \frac{d t}{t} \int_{\frac{1}{3}(1-t)}^{\frac{23}{80}-\frac{t}{8}} \frac{d u}{u(1-t-u)}+O(\varepsilon)\right) \\
& \geq(0.000516+0.010796+0.011351) \eta x \log ^{-1} x \\
& =0.022663 \eta x \log ^{-1} x \text {. }
\end{aligned}
$$


Using the above discussion and Lemma 20, we have

$$
\begin{aligned}
& \Omega_{19}+\Omega_{22}+\Omega_{25}+\Omega_{28}+\Omega_{31}+\Omega_{34}+\Omega_{37}+\Omega_{40}+\Omega_{42}+\Omega_{45} \\
& +\Omega_{48}+\Omega_{52}+\Omega_{56}+\Omega_{59}+\Omega_{62}+\Omega_{64}+\Omega_{65}+\Omega_{66}+\Omega_{68}+\Omega_{71} \\
& =\eta x \log ^{-1} x\left(\int_{\frac{3}{10}}^{\frac{19}{60}} \frac{d t}{t} \int_{\frac{57}{110}-\frac{12}{11} t}^{\frac{23}{80}-\frac{t}{8}} \frac{1}{u^{2}} w\left(\frac{1-t-u}{u}\right) d u\right. \\
& +\int_{\frac{19}{60}}^{\frac{247}{770}} \frac{d t}{t} \int_{\frac{19}{110}}^{\frac{23}{80}-\frac{t}{8}} \frac{1}{u^{2}} w\left(\frac{1-t-u}{u}\right) d u+\int_{\frac{247}{770}}^{\frac{359}{1100}} \frac{d t}{t} \int_{\frac{19}{110}}^{\frac{25}{44}-t} \frac{1}{u^{2}} w\left(\frac{1-t-u}{u}\right) d u \\
& +\int_{\frac{359}{1100}}^{\frac{481}{1450}} \frac{d t}{t} \int_{\frac{19}{110}}^{\frac{11}{5}-6 t} \frac{1}{u^{2}} w\left(\frac{1-t-u}{u}\right) d u \\
& +\int_{\frac{481}{1450}}^{\frac{443}{1305}} \frac{d t}{t} \int_{\frac{19}{110}}^{\frac{157}{290}-t} \frac{1}{u^{2}} w\left(\frac{1-t-u}{u}\right) d u \\
& +\int_{\frac{443}{1305}}^{\frac{19}{55}} \frac{d t}{t} \int_{\frac{19}{110}}^{\frac{29}{110}-\frac{2}{11} t} \frac{1}{u^{2}} w\left(\frac{1-t-u}{u}\right) d u \\
& +\int_{\frac{19}{55}}^{\frac{2143}{5620}} \frac{d t}{t} \int_{\frac{57}{98}-\frac{58}{49} t}^{\frac{29}{110}-\frac{2}{11} t} \frac{1}{u^{2}} w\left(\frac{1-t-u}{u}\right) d u \\
& +\int_{\frac{2143}{5620}}^{\frac{3963}{9860}} \frac{d t}{t} \int_{\frac{57}{98}-\frac{58}{49} t}^{\frac{59}{76}-\frac{29}{19} t} \frac{1}{u^{2}} w\left(\frac{1-t-u}{u}\right) d u \\
& \left.+\int_{\frac{3963}{9860}}^{\frac{4331}{9860}} \frac{d t}{t} \int_{\frac{9}{85}}^{\frac{59}{76}-\frac{29}{19} t} \frac{1}{u^{2}} w\left(\frac{1-t-u}{u}\right) d u+O(\varepsilon)\right) \\
& \geq \eta x \log ^{-1} x\left(\int_{\frac{3}{10}}^{\frac{19}{60}} \frac{d t}{t} \int_{\frac{57}{110}-\frac{12}{11} t}^{\frac{1}{3}(1-t)} \frac{1}{u(1-t-u)}\left(1+\log \left(\frac{1-t}{u}-2\right)\right) d u\right. \\
& +\int_{\frac{3}{10}}^{\frac{19}{60}} \frac{d t}{t} \int_{\frac{1}{3}(1-t)}^{\frac{23}{80}-\frac{t}{8}} \frac{d u}{u(1-t-u)}
\end{aligned}
$$




$$
\begin{aligned}
& +\int_{\frac{19}{60}}^{\frac{247}{770}} \frac{d t}{t} \int_{\frac{19}{110}}^{\frac{1}{3}(1-t)} \frac{1}{u(1-t-u)}\left(1+\log \left(\frac{1-t}{u}-2\right)\right) d u \\
& +\int_{\frac{19}{60}}^{\frac{247}{770}} \frac{d t}{t} \int_{\frac{1}{3}(1-t)}^{\frac{23}{80}-\frac{t}{8}} \frac{d u}{u(1-t-u)} \\
& +\int_{\frac{247}{770}}^{\frac{359}{1100}} \frac{d t}{t} \int_{\frac{19}{110}}^{\frac{1}{3}(1-t)} \frac{1}{u(1-t-u)}\left(1+\log \left(\frac{1-t}{u}-2\right)\right) d u \\
& +\int_{\frac{247}{770}}^{\frac{359}{1100}} \frac{d t}{t} \int_{\frac{1}{3}(1-t)}^{\frac{25}{44}-t} \frac{d u}{u(1-t-u)} \\
& +\int_{\frac{359}{1100}}^{\frac{28}{85}} \frac{d t}{t} \int_{\frac{19}{110}}^{\frac{1}{3}(1-t)} \frac{1}{u(1-t-u)}\left(1+\log \left(\frac{1-t}{u}-2\right)\right) d u \\
& +\int_{\frac{359}{1100}}^{\frac{28}{85}} \frac{d t}{t} \int_{\frac{1}{3}(1-t)}^{\frac{11}{5}-6 t} \frac{d u}{u(1-t-u)} \\
& +\int_{\frac{28}{85}}^{\frac{481}{1450}} \frac{d t}{t} \int_{\frac{19}{110}}^{\frac{11}{5}-6 t} \frac{1}{u(1-t-u)}\left(1+\log \left(\frac{1-t}{u}-2\right)\right) d u \\
& +\int_{\frac{481}{1450}}^{\frac{443}{1305}} \frac{d t}{t} \int_{\frac{19}{110}}^{\frac{157}{290}-t} \frac{1}{u(1-t-u)}\left(1+\log \left(\frac{1-t}{u}-2\right)\right) d u \\
& +\int_{\frac{443}{1305}}^{\frac{19}{55}} \frac{d t}{t} \int_{\frac{19}{110}}^{\frac{29}{110}-\frac{2}{11} t} \frac{1}{u(1-t-u)}\left(1+\log \left(\frac{1-t}{u}-2\right)\right) d u \\
& +\int_{\frac{19}{55}}^{\frac{65}{183}} \frac{d t}{t} \int_{\frac{57}{98}-\frac{58}{49} t}^{\frac{29}{110}-\frac{2}{11} t} \frac{1}{u(1-t-u)}\left(1+\log \left(\frac{1-t}{u}-2\right)\right) d u \\
& +0.5607 \int_{\frac{65}{183}}^{\frac{2143}{5620}} \frac{d t}{t} \int_{\frac{57}{98}-\frac{58}{49} t}^{\frac{1}{4}(1-t)} \frac{d u}{u^{2}}
\end{aligned}
$$




$$
\begin{aligned}
& +\int_{\frac{65}{183}}^{\frac{2143}{5620}} \frac{d t}{t} \int_{\frac{1}{4}(1-t)}^{\frac{29}{110}-\frac{2}{11} t} \frac{1}{u(1-t-u)}\left(1+\log \left(\frac{1-t}{u}-2\right)\right) d u \\
& +0.5607 \int_{\frac{2143}{5620}}^{\frac{3963}{9860}} \frac{d t}{t} \int_{\frac{57}{98}-\frac{58}{49} t}^{\frac{1}{4}(1-t)} \frac{d u}{u^{2}} \\
& +\int_{\frac{2143}{5620}}^{\frac{3963}{9860}} \frac{d t}{t} \int_{\frac{1}{4}(1-t)}^{\frac{59}{76}-\frac{29}{19} t} \frac{1}{u(1-t-u)}\left(1+\log \left(\frac{1-t}{u}-2\right)\right) d u \\
& +0.5607 \int_{\frac{3963}{9860}}^{\frac{40}{97}} \frac{d t}{t} \int_{\frac{9}{85}}^{\frac{1}{4}(1-t)} \frac{d u}{u^{2}} \\
& +\int_{\frac{3963}{9860}}^{\frac{40}{97}} \frac{d t}{t} \int_{\frac{1}{4}(1-t)}^{\frac{59}{76}-\frac{29}{19} t} \frac{1}{u(1-t-u)}\left(1+\log \left(\frac{1-t}{u}-2\right)\right) d u \\
& \left.+0.5607 \int_{\frac{40}{97}}^{\frac{4331}{9860}} \frac{d t}{t} \int_{\frac{9}{85}}^{\frac{59}{76}-\frac{29}{19} t} \frac{d u}{u^{2}}+O(\varepsilon)\right) \\
& \geq(0.034609+0.009183+0.009870+0.002518 \\
& +0.012892+0.003183+0.006792+0.000789 \\
& +0.004547+0.012016+0.008083+0.015437 \\
& +0.021797+0.049656+0.056577+0.028631 \\
& +0.038798+0.004151+0.052251) \eta x \log ^{-1} x \\
& =0.371780 \eta x \log ^{-1} x \text {. }
\end{aligned}
$$

The proof of Lemma 24 is complete.

LEMMA 25.

$$
\begin{aligned}
\Phi= & \Omega_{70}+\Omega_{73}+\Omega_{74}+\Omega_{76}+\Omega_{77}+\Omega_{79}+\Omega_{80}+\Omega_{81}+\Omega_{82}+\Omega_{83} \\
& +\Omega_{84}+\Omega_{85}+\Omega_{86}+\Omega_{87}+\Omega_{88}+\Omega_{89}+\Omega_{91}+\Omega_{93}+\Omega_{94} \\
\geq & 0.321616 \eta x \log ^{-1} x .
\end{aligned}
$$

Proof. On applying Lemmas 8, 19, 20 and 21, in the same way as in Lemma 24, we have 


$$
\begin{aligned}
& \Phi=\eta x \log ^{-1} x\left(\int_{\frac{19}{45}}^{\frac{19}{44}} \frac{d t}{t} \int_{\frac{57}{40}-\frac{29}{10} t}^{\frac{29}{100}-\frac{t}{5}} \frac{1}{u^{2}} w\left(\frac{1-t-u}{u}\right) d u\right. \\
& +\int_{\frac{19}{44}}^{\frac{897}{1972}} \frac{d t}{t} \int_{\frac{57}{40}-\frac{29}{10} t}^{\frac{29}{100}-\frac{t}{5}} \frac{1}{u^{2}} w\left(\frac{1-t-u}{u}\right) d u+\int_{\frac{19}{44}}^{\frac{897}{1972}} \frac{d t}{t} \int_{\frac{19}{25}-\frac{6}{5} t}^{\frac{13}{70}+\frac{t}{7}} \frac{1}{u^{2}} w\left(\frac{1-t-u}{u}\right) d u \\
& +\int_{\frac{897}{1972}}^{\frac{133}{290}} \frac{d t}{t} \int_{\frac{9}{85}}^{\frac{29}{100}} \frac{1}{u^{2}} w\left(\frac{1-t-u}{u}\right) d u+\int_{\frac{897}{1972}}^{\frac{133}{290}} \frac{d t}{t} \int_{\frac{19}{25}-\frac{6}{5} t}^{\frac{13}{70}+\frac{t}{7}} \frac{1}{u^{2}} w\left(\frac{1-t-u}{u}\right) d u \\
& +\int_{\frac{133}{290}}^{\frac{19}{40}} \frac{d t}{t} \int_{\frac{9}{85}}^{\frac{13}{70}+\frac{t}{7}} \frac{1}{u^{2}} w\left(\frac{1-t-u}{u}\right) d u+\int_{\frac{19}{40}}^{\frac{53}{110}} \frac{d t}{t} \int_{\frac{9}{85}}^{\frac{51}{70}-t} \frac{1}{u^{2}} w\left(\frac{1-t-u}{u}\right) d u \\
& +\int_{\frac{53}{110}}^{\frac{339}{700}} \frac{d t}{t} \int_{\frac{9}{85}}^{\frac{59}{18}-\frac{58}{9} t} \frac{1}{u^{2}} w\left(\frac{1-t-u}{u}\right) d u+\int_{\frac{53}{110}}^{\frac{339}{700}} \frac{d t}{t} \int_{\frac{19}{110}}^{\frac{51}{70}-t} \frac{1}{u^{2}} w\left(\frac{1-t-u}{u}\right) d u \\
& +\int_{\frac{339}{700}}^{\frac{49}{100}} \frac{d t}{t} \int_{\frac{9}{85}}^{\frac{59}{18}-\frac{58}{9} t} \frac{1}{u^{2}} w\left(\frac{1-t-u}{u}\right) d u+\int_{\frac{339}{700}}^{\frac{49}{100}} \frac{d t}{t} \int_{\frac{19}{110}}^{\frac{63}{20}-6 t} \frac{1}{u^{2}} w\left(\frac{1-t-u}{u}\right) d u \\
& \left.+\int_{\frac{49}{100}}^{\frac{1}{2}} \frac{d t}{t} \int_{\frac{19}{110}}^{\frac{7}{10}-t} \frac{1}{u^{2}} w\left(\frac{1-t-u}{u}\right) d u+O(\varepsilon)\right) \\
& \geq \eta x \log ^{-1} x\left(\int_{\frac{19}{45}}^{\frac{131}{308}} \frac{d t}{t} \int_{\frac{57}{40}-\frac{29}{10} t}^{\frac{29}{100}-\frac{t}{5}} \frac{d u}{u(1-t-u)}\right. \\
& +\int_{\frac{131}{308}}^{\frac{19}{44}} \frac{d t}{t} \int_{\frac{57}{40}-\frac{29}{10} t}^{\frac{1}{3}(1-t)} \frac{1}{u(1-t-u)}\left(1+\log \left(\frac{1-t}{u}-2\right)\right) d u \\
& +\int_{\frac{131}{308}}^{\frac{19}{44}} \frac{d t}{t} \int_{\frac{1}{3}(1-t)}^{\frac{29}{100}-\frac{t}{5}} \frac{d u}{u(1-t-u)} \\
& +\int_{\frac{19}{44}}^{\frac{47}{106}} \frac{d t}{t} \int_{\frac{57}{40}-\frac{29}{10} t}^{\frac{1}{3}(1-t)} \frac{1}{u(1-t-u)}\left(1+\log \left(\frac{1-t}{u}-2\right)\right) d u \\
& +\int_{\frac{19}{44}}^{\frac{47}{106}} \frac{d t}{t} \int_{\frac{1}{3}(1-t)}^{\frac{29}{100}-\frac{t}{5}} \frac{d u}{u(1-t-u)}+0.5607 \int_{\frac{47}{106}}^{\frac{897}{1972}} \frac{d t}{t} \int_{\frac{57}{40}-\frac{29}{10} t}^{\frac{1}{4}(1-t)} \frac{d u}{u^{2}}
\end{aligned}
$$




$$
\begin{aligned}
& +\int_{\frac{47}{106}}^{\frac{897}{1972}} \frac{d t}{t} \int_{\frac{1}{4}(1-t)}^{\frac{1}{3}(1-t)} \frac{1}{u(1-t-u)}\left(1+\log \left(\frac{1-t}{u}-2\right)\right) d u \\
& +\int_{\frac{47}{106}}^{\frac{897}{1972}} \frac{d t}{t} \int_{\frac{1}{3}(1-t)}^{\frac{29}{100}-\frac{t}{5}} \frac{d u}{u(1-t-u)}+\int_{\frac{19}{44}}^{\frac{897}{1972}} \frac{d t}{t} \int_{\frac{19}{25}-\frac{6}{5} t}^{\frac{13}{70}+\frac{t}{7}} \frac{d u}{u(1-t-u)} \\
& +0.5607 \int_{\frac{897}{1972}}^{\frac{133}{290}} \frac{d t}{t} \int_{\frac{9}{85}}^{\frac{1}{4}(1-t)} \frac{d u}{u^{2}} \\
& +\int_{\frac{897}{1972}}^{\frac{133}{290}} \frac{d t}{t} \int_{\frac{1}{4}(1-t)}^{\frac{1}{3}(1-t)} \frac{1}{u(1-t-u)}\left(1+\log \left(\frac{1-t}{u}-2\right)\right) d u \\
& +\int_{\frac{897}{1972}}^{\frac{133}{290}} \frac{d t}{t} \int_{\frac{1}{3}(1-t)}^{\frac{29}{100}-\frac{t}{5}} \frac{d u}{u(1-t-u)}+\int_{\frac{897}{1972}}^{\frac{133}{290}} \frac{d t}{t} \int_{\frac{19}{25}-\frac{6}{5} t}^{\frac{13}{70}+\frac{t}{7}} \frac{d u}{u(1-t-u)} \\
& +0.5607 \int_{\frac{133}{290}}^{\frac{19}{40}} \frac{d t}{t} \int_{\frac{9}{85}}^{\frac{1}{4}(1-t)} \frac{d u}{u^{2}} \\
& +\int_{\frac{133}{290}}^{\frac{19}{40}} \frac{d t}{t} \int_{\frac{1}{4}(1-t)}^{\frac{1}{3}(1-t)} \frac{1}{u(1-t-u)}\left(1+\log \left(\frac{1-t}{u}-2\right)\right) d u \\
& +\int_{\frac{133}{290}}^{\frac{19}{40}} \frac{d t}{t} \int_{\frac{1}{3}(1-t)}^{\frac{13}{70}+\frac{t}{7}} \frac{d u}{u(1-t-u)}+0.5607 \int_{\frac{19}{40}}^{\frac{53}{110}} \frac{d t}{t} \int_{\frac{9}{85}}^{\frac{1}{4}(1-t)} \frac{d u}{u^{2}} \\
& +\int_{\frac{19}{40}}^{\frac{53}{110}} \frac{d t}{t} \int_{\frac{1}{4}(1-t)}^{\frac{1}{3}(1-t)} \frac{1}{u(1-t-u)}\left(1+\log \left(\frac{1-t}{u}-2\right)\right) d u \\
& +\int_{\frac{19}{40}}^{\frac{53}{110}} \frac{d t}{t} \int_{\frac{1}{3}(1-t)}^{\frac{51}{70}-t} \frac{d u}{u(1-t-u)}+0.5607 \int_{\frac{53}{110}}^{\frac{339}{700}} \frac{d t}{t} \int_{\frac{9}{85}}^{\frac{1}{4}(1-t)} \frac{d u}{u^{2}} \\
& +\int_{\frac{53}{110}}^{\frac{339}{700}} \frac{d t}{t} \int_{\frac{1}{4}(1-t)}^{\frac{59}{18}-\frac{58}{9} t} \frac{1}{u(1-t-u)}\left(1+\log \left(\frac{1-t}{u}-2\right)\right) d u \\
& +\int_{\frac{53}{110}}^{\frac{339}{700}} \frac{d t}{t} \int_{\frac{19}{110}}^{\frac{51}{70}-t} \frac{d u}{u(1-t-u)}+0.5607 \int_{\frac{339}{700}}^{\frac{109}{223}} \frac{d t}{t} \int_{\frac{9}{85}}^{\frac{1}{4}(1-t)} \frac{d u}{u^{2}}
\end{aligned}
$$




$$
\begin{aligned}
& +\int_{\frac{339}{700}}^{\frac{109}{223}} \frac{d t}{t} \int_{\frac{1}{4}(1-t)}^{\frac{59}{18}-\frac{58}{9} t} \frac{1}{u(1-t-u)}\left(1+\log \left(\frac{1-t}{u}-2\right)\right) d u \\
& +0.5607 \int_{\frac{109}{223}}^{\frac{49}{100}} \frac{d t}{t} \int_{\frac{9}{85}}^{\frac{59}{18}-\frac{58}{9} t} \frac{d u}{u^{2}}+\int_{\frac{339}{700}}^{\frac{49}{100}} \frac{d t}{t} \int_{\frac{19}{110}}^{\frac{63}{20}-6 t} \frac{d u}{u(1-t-u)} \\
& \left.+\int_{\frac{49}{100}}^{\frac{1}{2}} \frac{d t}{t} \int_{\frac{19}{110}}^{\frac{7}{10}-t} \frac{d u}{u(1-t-u)}+O(\varepsilon)\right) \\
& \geq(0.000892+0.001922+0.002834+0.015864 \\
& +0.005547+0.013560+0.025626+0.006139 \\
& +0.014504+0.009587+0.008357+0.002151 \\
& +0.004454+0.038219+0.036373+0.038613 \\
& +0.014188+0.015100+0.016708+0.004887 \\
& +0.004744+0.005821+0.008584+0.003871 \\
& +0.001900+0.010238+0.010933) \eta x \log ^{-1} x \\
& =0.321616 \eta x \log ^{-1} x \text {. }
\end{aligned}
$$

The proof of Lemma 25 is complete.

Lemma 26.

$$
\begin{aligned}
\Omega_{5}+\Omega_{7}+\Omega_{9}+ & \Omega_{11}+\Omega_{13}+\Omega_{15}+\Omega_{18}+\Omega_{21}+\Omega_{24}+\Omega_{27}+\Omega_{30} \\
& +\Omega_{33}+\Omega_{36}+\Omega_{39}+\Omega_{41}+\Omega_{43}+\Omega_{46}+\Omega_{49}+\Omega_{53} \\
\geq & 0.277632 \eta x \log ^{-1} x .
\end{aligned}
$$

Proof. We have

(25)

$$
\begin{aligned}
\Omega_{5}= & \sum_{X^{\frac{133}{580}}<p \leq X^{\frac{19}{80}}} \sum_{p^{-1} X^{\frac{133}{290}}<q<p} S\left(\mathcal{A}_{p q}, q\right) \\
= & \sum_{X^{\frac{133}{580}}<p \leq X^{\frac{19}{80}}} \sum_{p^{-1} X^{\frac{133}{290}}<q<p} S\left(\mathcal{A}_{p q}, X^{\delta}\right) \\
& -\sum_{X^{\frac{113}{580}<p \leq X^{\frac{19}{80}}}} \sum_{p^{-1} X^{\frac{133}{290}}<q<p} \sum_{X^{\delta}<r<X^{\frac{9}{85}}} S\left(\mathcal{A}_{p q r}, r\right) \\
& -\sum_{X^{\frac{133}{580}<p \leq X^{\frac{19}{80}}}} \sum_{p^{-1} X^{\frac{133}{290}}<q<p} S\left(\mathcal{A}_{p q r}, r\right) \\
= & \Phi_{1}-\Phi_{2}-\Phi_{3} .
\end{aligned}
$$

Let $z=X^{\delta}$ and $D=D(p, q)=X^{\frac{21}{40}} /(p q)$. Applying Iwaniec's sieve 
method, we have

$$
\begin{aligned}
\Phi_{1} \leq & \frac{\eta x}{\log z} \sum_{X^{\frac{133}{580}<p \leq X^{\frac{19}{80}}}} \sum_{p^{-1} X^{\frac{133}{290}<q<p}} \frac{1}{p q} F\left(\frac{\log D}{\log z}\right) \\
& +\sum_{X^{\frac{133}{580}<p \leq X^{\frac{19}{80}}}} \sum_{p^{-1} X_{X^{\frac{133}{290}}<q<p}} \sum_{r<X^{\frac{21}{40}} /(p q)} a(r) \widetilde{r}(\mathcal{A}, p q r) \\
& +O\left(\varepsilon \eta x \log ^{-1} x\right) .
\end{aligned}
$$

Let $m=p q r$. Then Lemma 16 yields

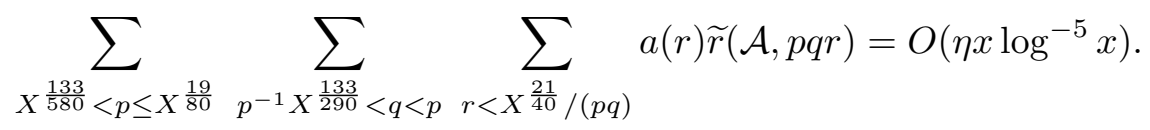

Hence,

$$
\begin{aligned}
& \Phi_{1} \leq \frac{e^{-\gamma}}{\delta} \eta x \log ^{-1} x \sum_{X^{\frac{133}{580}<p \leq X} X^{\frac{19}{80}}} \sum_{p^{-1} X^{\frac{133}{290}}<q<p} \frac{1}{p q}+O\left(\delta \eta x \log ^{-1} x\right) \\
& =\eta \sum_{X^{\frac{133}{580}<p \leq X} X^{\frac{19}{80}}} \sum_{p^{-1} X^{\frac{133}{290}}<q<p} S\left(\mathcal{B}_{p q}, X^{\delta}\right)+O\left(\delta \eta x \log ^{-1} x\right) .
\end{aligned}
$$

In the same way, we can get the lower bound

$$
\Phi_{1} \geq \eta \sum_{X^{\frac{133}{580}}<p \leq X^{\frac{19}{80}}} \sum_{p^{-1} X^{\frac{133}{290}}<q<p} S\left(\mathcal{B}_{p q}, X^{\delta}\right)+O\left(\delta \eta x \log ^{-1} x\right) .
$$

Now we have the asymptotic formula

$$
\Phi_{1}=\eta \sum_{X^{\frac{133}{580}}<p \leq X^{\frac{19}{80}}} \sum_{p^{-1} X^{\frac{133}{290}}<q<p} S\left(\mathcal{B}_{p q}, X^{\delta}\right)+O\left(\delta \eta x \log ^{-1} x\right) .
$$

Using Lemma 18, we have

$$
\begin{aligned}
\Phi_{2}= & \sum_{\substack{X^{\frac{133}{580}}<p \leq X^{\frac{19}{80}} \\
p^{-1}}} \sum_{X^{\frac{133}{290}}<q<p} \sum_{X^{\delta}<r<X^{\frac{9}{85}}} S\left(\mathcal{B}_{p q r}, r\right) \\
& +O\left(\delta \eta x \log ^{-1} x\right) .
\end{aligned}
$$

By Lemma 13 with the region (i), (7) holds. Then Lemma 19 with a small modification yields

$$
\begin{aligned}
\Phi_{3}= & \sum_{\substack{X^{\frac{133}{580}}<p \leq X^{\frac{19}{80}} \\
p^{-1} X^{\frac{133}{290}<q<p}}} \sum_{X^{\frac{9}{85}<r<\min \left(q,\left(\frac{2 X}{p q}\right)^{\frac{1}{2}}\right)}} S\left(\mathcal{B}_{p q r}, r\right) \\
& +O\left(\delta \eta x \log ^{-1} x\right) .
\end{aligned}
$$


Gathering together (25)-(28), we have

$$
\begin{aligned}
\Omega_{5}= & \sum_{X^{\frac{133}{580}}<p \leq X} \sum_{X^{\frac{19}{80}}} S\left(\mathcal{B}_{p q}, q\right)+O\left(\delta \eta x \log ^{-1} x\right) \\
= & \eta x \log ^{-1} x \int_{\frac{133}{580}}^{\frac{1933}{80}} \frac{d t}{t} \int_{\frac{133}{290}-t}^{t} \frac{1}{u^{2}} w\left(\frac{1-t-u}{u}\right) d u+O\left(\delta \eta x \log ^{-1} x\right) \\
= & \eta x \log ^{-1} x \int_{\frac{133}{580}}^{\frac{19}{t}} \frac{d t}{t} \int_{\frac{133}{290}-t}^{t} \frac{1}{u(1-t-u)}\left(1+\log \left(\frac{1-t}{u}-2\right)\right) d u \\
& +O\left(\delta \eta x \log ^{-1} x\right) \\
\geq & 0.003001 \eta x \log ^{-1} x .
\end{aligned}
$$

In the same way, it can be shown that

$$
\begin{aligned}
& \Omega_{7}+\Omega_{9}+\Omega_{11}+\Omega_{13}+\Omega_{15}+\Omega_{18}+\Omega_{21}+\Omega_{24}+\Omega_{27}+\Omega_{30} \\
& +\Omega_{33}+\Omega_{36}+\Omega_{39}+\Omega_{41}+\Omega_{43}+\Omega_{46}+\Omega_{49}+\Omega_{53} \\
& =\eta x \log ^{-1} x\left(\int_{\frac{19}{80}}^{\frac{53}{220}} \frac{d t}{t} \int_{\frac{133}{290}-t}^{\frac{19}{40}-t} \frac{1}{u^{2}} w\left(\frac{1-t-u}{u}\right) d u\right. \\
& +\int_{\frac{53}{220}}^{\frac{151}{580}} \frac{d t}{t} \int_{\frac{133}{290}-t}^{\frac{53}{110}-t} \frac{1}{u^{2}} w\left(\frac{1-t-u}{u}\right) d u+\int_{\frac{151}{580}}^{\frac{17}{55}} \frac{d t}{t} \int_{\frac{897}{1972}-t}^{\frac{53}{110}-t} \frac{1}{u^{2}} w\left(\frac{1-t-u}{u}\right) d u \\
& +\int_{\frac{17}{55}}^{\frac{3441}{9860}} \frac{d t}{t} \int_{\frac{897}{1972}-t}^{\frac{59}{134}-\frac{58}{67} t} \frac{1}{u^{2}} w\left(\frac{1-t-u}{u}\right) d u+\int_{\frac{3441}{9860}}^{\frac{37}{100}} \frac{d t}{t} \int_{\frac{9}{85}}^{\frac{59}{134}-\frac{58}{67} t} \frac{1}{u^{2}} w\left(\frac{1-t-u}{u}\right) d u \\
& \left.+\int_{\frac{37}{100}}^{\frac{653}{1700}} \frac{d t}{t} \int_{\frac{9}{85}}^{\frac{49}{100}-t} \frac{1}{u^{2}} w\left(\frac{1-t-u}{u}\right) d u+O(\varepsilon)\right) \\
& \geq \eta x \log ^{-1} x\left(\int_{\frac{19}{80}}^{\frac{53}{220}} \frac{d t}{t} \int_{\frac{133}{290}-t}^{\frac{19}{40}-t} \frac{1}{u(1-t-u)}\left(1+\log \left(\frac{1-t}{u}-2\right)\right) d u\right. \\
& +\int_{\frac{53}{220}}^{\frac{151}{580}} \frac{d t}{t} \int_{\frac{133}{290}-t}^{\frac{53}{110}-t} \frac{1}{u(1-t-u)}\left(1+\log \left(\frac{1-t}{u}-2\right)\right) d u
\end{aligned}
$$




$$
\begin{aligned}
& +\int_{\frac{151}{580}}^{\frac{404}{1479}} \frac{d t}{t} \int_{\frac{897}{1972}-t}^{\frac{53}{110}-t} \frac{1}{u(1-t-u)}\left(1+\log \left(\frac{1-t}{u}-2\right)\right) d u \\
& +0.5607 \int_{\frac{404}{1479}}^{\frac{17}{55}} \frac{d t}{t} \int_{\frac{897}{1972}-t}^{\frac{1}{4}(1-t)} \frac{d u}{u^{2}} \\
& +\int_{\frac{404}{1479}}^{\frac{17}{55}} \frac{d t}{t} \int_{\frac{1}{4}(1-t)}^{\frac{53}{110}-t} \frac{1}{u(1-t-u)}\left(1+\log \left(\frac{1-t}{u}-2\right)\right) d u \\
& +0.5607 \int_{\frac{17}{55}}^{\frac{3441}{9860}} \frac{d t}{t} \int_{\frac{897}{1972}-t}^{\frac{59}{134}-\frac{58}{67} t} \frac{d u}{u^{2}}+0.5607 \int_{\frac{3441}{9860}}^{\frac{37}{100}} \frac{d t}{t} \int_{\frac{9}{85}}^{\frac{59}{134}-\frac{58}{67} t} \frac{d u}{u^{2}} \\
& \left.+0.5607 \int_{\frac{37}{100}}^{\frac{653}{1700}} \frac{d t}{t} \int_{\frac{9}{85}}^{\frac{49}{100}-t} \frac{d u}{u^{2}}+O(\varepsilon)\right) \\
& \geq(0.002490+0.020848+0.018080+0.033046 \\
& +0.027398+0.105118+0.055429+0.012222) \eta x \log ^{-1} x \\
& =0.274631 \eta x \log ^{-1} x \text {. }
\end{aligned}
$$

The proof of Lemma 26 is complete.

LEMma 27.

$$
\begin{aligned}
\Phi & =\Omega_{44}+\Omega_{47}+\Omega_{51}+\Omega_{55}+\Omega_{58}+\Omega_{61}+\Omega_{63}+\Omega_{67}+\Omega_{69}+\Omega_{72} \\
& \geq 0.033767 \eta x \log ^{-1} x .
\end{aligned}
$$

Proof. On applying Lemmas 11, 19, 20 and 21, in the same way as in Lemma 24, we have

$$
\begin{aligned}
\Phi= & \eta x \log ^{-1} x\left(\int_{\frac{1843}{5280}}^{\frac{37}{100}} \frac{d t}{t} \int_{\frac{171}{295}-\frac{70}{59} t}^{\frac{57}{98}-\frac{58}{49} t} \frac{1}{u^{2}} w\left(\frac{1-t-u}{u}\right) d u\right. \\
& +\int_{\frac{37}{100}}^{\frac{5541}{13940}} \frac{d t}{t} \int_{\frac{133}{230}-\frac{82}{69} t}^{\frac{57}{49}-\frac{58}{49} t} \frac{1}{u^{2}} w\left(\frac{1-t-u}{u}\right) d u \\
& +\int_{\frac{5541}{13940}}^{\frac{3963}{9860}} \frac{d t}{t} \int_{\frac{9}{85}}^{\frac{57}{98}-\frac{58}{49} t} \frac{1}{u^{2}} w\left(\frac{1-t-u}{u}\right) d u
\end{aligned}
$$




$$
\begin{aligned}
& +\int_{\frac{1063}{2640}}^{\frac{19}{45}} \frac{d t}{t} \int_{\frac{59}{76}-\frac{29}{19} t}^{\frac{179}{230}-\frac{35}{23} t} \frac{1}{u^{2}} w\left(\frac{1-t-u}{u}\right) d u \\
& \left.+\int_{\frac{19}{45}}^{\frac{4331}{9860}} \frac{d t}{t} \int_{\frac{59}{76}-\frac{29}{19} t}^{\frac{421}{540}-\frac{41}{27} t} \frac{1}{u^{2}} w\left(\frac{1-t-u}{u}\right) d u+O(\varepsilon)\right) \\
& \geq \eta x \log ^{-1} x\left(\int_{\frac{1843}{5280}}^{\frac{389}{1105}} \frac{d t}{t} \int_{\frac{171}{295}-\frac{70}{59} t}^{\frac{57}{98}-\frac{58}{49} t} \frac{1}{u(1-t-u)}\left(1+\log \left(\frac{1-t}{u}-2\right)\right) d u\right. \\
& +0.5607 \int_{\frac{389}{105}}^{\frac{65}{183}} \frac{d t}{t} \int_{\frac{171}{295}-\frac{70}{59} t}^{\frac{1}{4}(1-t)} \frac{d u}{u^{2}} \\
& +\int_{\frac{389}{105}}^{\frac{65}{183}} \frac{d t}{t} \int_{\frac{1}{4}(1-t)}^{\frac{57}{98}-\frac{58}{49} t} \frac{1}{u(1-t-u)}\left(1+\log \left(\frac{1-t}{u}-2\right)\right) d u \\
& +0.5607 \int_{\frac{65}{183}}^{\frac{37}{100}} \frac{d t}{t} \int_{\frac{171}{295}-\frac{70}{59} t}^{\frac{57}{98}-\frac{58}{49} t} \frac{d u}{u^{2}}+0.5607 \int_{\frac{37}{100}}^{\frac{5541}{13940}} \frac{d t}{t} \int_{\frac{133}{230}-\frac{82}{69} t}^{\frac{57}{98}-\frac{58}{49} t} \frac{d u}{u^{2}} \\
& +0.5607 \int_{\frac{5541}{13940}}^{\frac{3963}{9860}} \frac{d t}{t} \int_{\frac{9}{85}}^{\frac{57}{98}-\frac{58}{49} t} \frac{d u}{u^{2}} \\
& +\int_{\frac{1063}{2640}}^{\frac{40}{97}} \frac{d t}{t} \int_{\frac{59}{76}-\frac{29}{19} t}^{\frac{179}{230}-\frac{35}{23} t} \frac{1}{u(1-t-u)}\left(1+\log \left(\frac{1-t}{u}-2\right)\right) d u \\
& +0.5607 \int_{\frac{40}{97}}^{\frac{27}{65}} \frac{d t}{t} \int_{\frac{59}{76}-\frac{29}{19} t}^{\frac{1}{4}(1-t)} \frac{d u}{u^{2}} \\
& +\int_{\frac{40}{97}}^{\frac{27}{65}} \frac{d t}{t} \int_{\frac{1}{4}(1-t)}^{\frac{179}{230}-\frac{35}{23} t} \frac{1}{u(1-t-u)}\left(1+\log \left(\frac{1-t}{u}-2\right)\right) d u \\
& +0.5607 \int_{\frac{27}{65}}^{\frac{19}{45}} \frac{d t}{t} \int_{\frac{59}{76}-\frac{29}{19} t}^{\frac{179}{230}-\frac{35}{23} t} \frac{d u}{u^{2}} \\
& \left.+0.5607 \int_{\frac{19}{45}}^{\frac{4331}{9860}} \frac{d t}{t} \int_{\frac{59}{76}-\frac{29}{19} t}^{\frac{421}{540}-\frac{41}{27} t} \frac{d u}{u^{2}}+O(\varepsilon)\right)
\end{aligned}
$$




$$
\begin{aligned}
\geq & (0.000517+0.000285+0.000279+0.002997 \\
& +0.013589+0.001413+0.002113+0.000371 \\
& +0.000359+0.001830+0.010014) \eta x \log ^{-1} x \\
= & 0.033767 \eta x \log ^{-1} x .
\end{aligned}
$$

The proof of Lemma 27 is complete.

LEMMA 28.

$$
\Phi=\Omega_{75}+\Omega_{78}+\Omega_{90}+\Omega_{92} \geq 0.019054 \eta x \log ^{-1} x .
$$

Proof. On applying Lemmas 12 and 19-21, in the same way as in Lemma 24, we have

$$
\begin{aligned}
& \Phi=\eta x \log ^{-1} x\left(\int_{\frac{4331}{9860}}^{\frac{2691}{5950}} \frac{d t}{t} \int_{\frac{57}{40}-\frac{35}{12} t}^{\frac{57}{40}-\frac{29}{10} t} \frac{1}{u^{2}} w\left(\frac{1-t-u}{u}\right) d u\right. \\
& +\int_{\frac{2691}{5950}}^{\frac{897}{1972}} \frac{d t}{t} \int_{\frac{9}{85}}^{\frac{57}{40}-\frac{29}{10} t} \frac{1}{u^{2}} w\left(\frac{1-t-u}{u}\right) d u \\
& +\int_{\frac{233}{480}}^{\frac{49}{100}} \frac{d t}{t} \int_{\frac{59}{18}-\frac{58}{9} t}^{\frac{179}{55}-\frac{70}{11} t} \frac{1}{u^{2}} w\left(\frac{1-t-u}{u}\right) d u \\
& \left.+\int_{\frac{49}{100}}^{\frac{2944}{5950}} \frac{d t}{t} \int_{\frac{9}{85}}^{\frac{179}{55}-\frac{70}{11} t} \frac{1}{u^{2}} w\left(\frac{1-t-u}{u}\right) d u+O(\varepsilon)\right) \\
& \geq \eta x \log ^{-1} x\left(\int_{\frac{4331}{9860}}^{\frac{141}{320}} \frac{d t}{t} \int_{\frac{57}{40}-\frac{35}{12} t}^{\frac{57}{40}-\frac{29}{10} t} \frac{1}{u(1-t-u)}\left(1+\log \left(\frac{1-t}{u}-2\right)\right) d u\right. \\
& +0.5607 \int_{\frac{141}{320}}^{\frac{47}{106}} \frac{d t}{t} \int_{\frac{57}{40}-\frac{35}{12} t}^{\frac{1}{4}(1-t)} \frac{d u}{u^{2}} \\
& +\int_{\frac{141}{320}}^{\frac{47}{106}} \frac{d t}{t} \int_{\frac{1}{4}(1-t)}^{\frac{57}{40}-\frac{29}{10} t} \frac{1}{u(1-t-u)}\left(1+\log \left(\frac{1-t}{u}-2\right)\right) d u \\
& +0.5607 \int_{\frac{47}{106}}^{\frac{2691}{5950}} \frac{d t}{t} \int_{\frac{57}{40}-\frac{35}{12} t}^{\frac{57}{40}-\frac{29}{10} t} \frac{d u}{u^{2}}+0.5607 \int_{\frac{2691}{5950}}^{\frac{897}{1972}} \frac{d t}{t} \int_{\frac{9}{85}}^{\frac{57}{40}-\frac{29}{10} t} \frac{d u}{u^{2}}
\end{aligned}
$$




$$
\begin{aligned}
& +\int_{\frac{233}{480}}^{\frac{109}{223}} \frac{d t}{t} \int_{\frac{59}{18}-\frac{58}{9} t}^{\frac{179}{55}-\frac{70}{11} t} \frac{1}{u(1-t-u)}\left(1+\log \left(\frac{1-t}{u}-2\right)\right) d u \\
& +0.5607 \int_{\frac{109}{223}}^{\frac{49}{100}} \frac{d t}{t} \int_{\frac{59}{18}-\frac{58}{9} t}^{\frac{1}{4}(1-t)} \frac{d u}{u^{2}} \\
& +\int_{\frac{109}{223}}^{\frac{49}{100}} \frac{d t}{t} \int_{\frac{1}{4}(1-t)}^{\frac{179}{55}-\frac{70}{11} t} \frac{1}{u(1-t-u)}\left(1+\log \left(\frac{1-t}{u}-2\right)\right) d u \\
& +0.5607 \int_{\frac{49}{100}}^{\frac{661}{1345}} \frac{d t}{t} \int_{\frac{9}{85}}^{\frac{1}{4}(1-t)} \frac{d u}{u^{2}} \\
& +\int_{\frac{49}{100}}^{\frac{661}{1345}} \frac{d t}{t} \int_{\frac{1}{4}(1-t)}^{\frac{179}{55}} \frac{1}{u(1-t-u)}\left(1+\log \left(\frac{1-t}{u}-2\right)\right) d u
\end{aligned}
$$

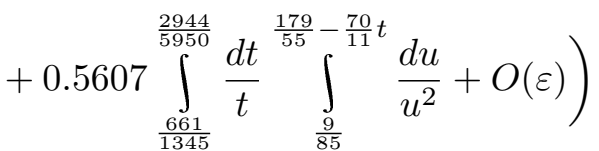

$$
\begin{aligned}
& \geq(0.000612+0.000691+0.000645+0.005586 \\
& +0.001031+0.002927+0.000331+0.000980 \\
& +0.002633+0.000435+0.003183) \eta x \log ^{-1} x \\
& =0.019054 \eta x \log ^{-1} x \text {. }
\end{aligned}
$$

The proof of Lemma 28 is complete.

LEMMA 29.

$$
\begin{aligned}
\Phi= & \Omega_{1}+\Omega_{2}+\Omega_{3}+\Omega_{4}+\Omega_{6}+\Omega_{8}+\Omega_{10}+\Omega_{12}+\Omega_{14}+\Omega_{17}+\Omega_{20} \\
& +\Omega_{23}+\Omega_{26}+\Omega_{29}+\Omega_{32}+\Omega_{35}+\Omega_{38}+\Omega_{50}+\Omega_{54}+\Omega_{57}+\Omega_{60} \\
\geq & 1.902585 \eta x \log ^{-1} x .
\end{aligned}
$$

Pr o of. The main idea in this lemma is adopted from [20]. We have

$$
\begin{aligned}
(29) \Omega_{1} \geq & \sum_{X^{\frac{9}{85}}<p \leq X^{\frac{21}{100}}-10^{-8}} \sum_{X^{\frac{9}{85}<q<p}} S\left(\mathcal{A}_{p q}, q\right) \\
= & \sum_{X^{\frac{9}{85}}<p \leq X^{\frac{21}{100}}-10^{-8}} \sum_{X^{\frac{9}{85}<q<p}} S\left(\mathcal{A}_{p q}, X^{\delta}\right) \\
& -\sum_{X^{\frac{9}{85}}<p \leq X^{\frac{21}{100}}-10^{-8}} \sum_{X^{\frac{9}{85}<q<p}} \sum_{X^{\delta}<r<X^{\frac{9}{85}}} S\left(\mathcal{A}_{p q r}, r\right)
\end{aligned}
$$




$$
\begin{aligned}
& -\sum_{\substack{X^{\frac{9}{85}}<p \leq X \frac{21}{100}-10^{-8} \\
X^{\frac{9}{85}<q<p}}} \sum_{X^{\frac{9}{85}<r<\min \left(q,\left(\frac{2 X}{p q}\right)^{\frac{1}{2}}\right)}} S\left(\mathcal{A}_{p q r}, r\right) \\
= & \Phi_{1}-\Phi_{2}-\Phi_{3} .
\end{aligned}
$$

Let $z=X^{\delta}$ and $D(p, q)=X^{\frac{21}{40}} /(p q)$. Using Iwaniec's sieve method, in the same way as in Lemma 26 , we have

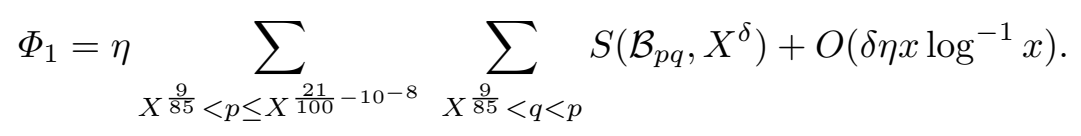

Lemma 18 yields

(31) $\Phi_{2}=\eta \sum_{X^{\frac{9}{85}}<p \leq X \frac{21}{100}-10^{-8}} \sum_{X^{\frac{9}{85}<q<p}} \sum_{X^{\delta}<r<X} S\left(\mathcal{B}_{p q r}, r\right)+O\left(\delta \eta x \log ^{-1} x\right)$.

Hence,

$$
\begin{aligned}
\Phi_{1}-\Phi_{2}= & \sum_{X^{\frac{9}{85}<p \leq X \frac{21}{100}-10^{-8}}} \sum_{X \frac{9}{85}<q<p} S\left(\mathcal{B}_{p q}, X^{\frac{9}{85}}\right)+O\left(\delta \eta x \log ^{-1} x\right) \\
= & \eta x \log ^{-1} x \cdot \frac{85}{9} \int_{\frac{9}{85}}^{\frac{21}{100}-10^{-8}} \frac{d t}{t} \int_{\frac{9}{85}}^{t} \frac{1}{u} w\left(\frac{85}{9}(1-t-u)\right) d u \\
& +O\left(\delta \eta x \log ^{-1} x\right) \\
\geq & \eta x \log ^{-1} x\left(\frac{85}{9} \int_{\frac{9}{85}}^{\frac{21}{100}} \frac{d t}{t} \int_{\frac{9}{85}}^{t} \frac{1}{u} w\left(\frac{85}{9}(1-t-u)\right) d u-10^{-6}\right) \\
\geq & \eta x \log ^{-1} x\left(0.5612 \cdot \frac{85}{9} \int_{\frac{9}{85}}^{\frac{21}{100}} \frac{d t}{t} \int_{\frac{9}{85}}^{t} \frac{d u}{u}-10^{-6}\right) \\
\geq & 1.242693 \eta x \log ^{-1} x .
\end{aligned}
$$

Note that if $q<(2 X / p)^{\frac{1}{3}}$, then $q<(2 X /(p q))^{\frac{1}{2}}$. We have

$$
\begin{aligned}
& \Phi_{3} \leq \sum_{X^{\frac{9}{85}<p \leq X \frac{21}{100}-10^{-8}}} \sum_{X^{\frac{9}{85}<q<p}} \sum_{X^{\frac{9}{85}<r<q}} S\left(\mathcal{A}_{p q r}, X^{\frac{9}{85}}\right)
\end{aligned}
$$

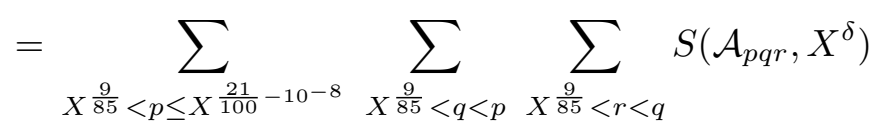

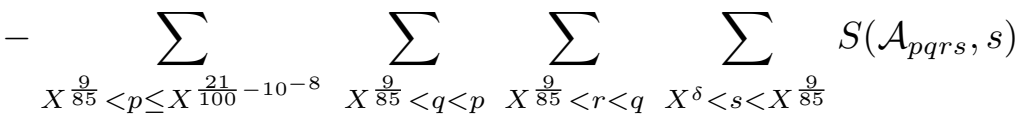

$$
\begin{aligned}
& =\Phi_{4}-\Phi_{5} \text {. }
\end{aligned}
$$


Let $z=X^{\delta}$ and $D(p, q, r)=X^{10^{-8}}$. Using Lemma 17 with region (i), in the same way as in Lemma 26, we have

$$
\begin{aligned}
\Phi_{4}= & \eta \sum_{X^{\frac{9}{85}}<p \leq X X^{\frac{21}{100}}-10^{-8}} \sum_{X^{\frac{9}{85}<q<p}} \sum_{X X^{\frac{9}{85}<r<q}} S\left(\mathcal{B}_{p q r}, X^{\delta}\right) \\
& +O\left(\delta \eta x \log ^{-1} x\right) .
\end{aligned}
$$

Lemma 18 yields

$$
\begin{aligned}
\Phi_{5}= & \sum_{\substack{X^{\frac{9}{85}}<p \leq X^{\frac{21}{100}}-10^{-8}\\
}} \sum_{X^{\frac{9}{85}<q<p}} \sum_{X^{\frac{9}{85}<r<q}} \sum_{X^{\delta}<s<X^{\frac{9}{85}}} S\left(\mathcal{B}_{\text {pqrs }}, s\right) \\
& +O\left(\delta \eta x \log ^{-1} x\right) .
\end{aligned}
$$

We therefore have

$$
\begin{aligned}
& \Phi_{3} \leq \eta \sum_{X^{\frac{9}{85}<p \leq X} X^{\frac{21}{100}-10^{-8}}} \sum_{X^{\frac{9}{85}<q<p}} \sum_{X^{\frac{9}{85}<r<q}} S\left(\mathcal{B}_{p q r}, X^{\frac{9}{85}}\right) \\
& +O\left(\delta \eta x \log ^{-1} x\right) \\
& \leq \eta x \log ^{-1} x \cdot \frac{85}{9} \int_{\frac{9}{85}}^{\frac{21}{100}} \frac{d t}{t} \int_{\frac{9}{85}}^{t} \frac{d u}{u} \int_{\frac{9}{85}}^{u} \frac{1}{v} w\left(\frac{85}{9}(1-t-u-v)\right) d v \\
& \leq \eta x \log ^{-1} x\left(0.5617 \cdot \frac{85}{9} \int_{\frac{9}{85}}^{\frac{49}{255}} \frac{d t}{t} \int_{\frac{9}{85}}^{t} \frac{d u}{u} \int_{\frac{9}{85}}^{u} \frac{d v}{v}\right. \\
& +0.5617 \cdot \frac{85}{9} \int_{\frac{49}{255}}^{\frac{21}{100}} \frac{d t}{t} \int_{\frac{9}{85}}^{\frac{1}{2}\left(\frac{49}{85}-t\right)} \frac{d u}{u} \int_{\frac{9}{85}}^{u} \frac{d v}{v} \\
& \left.+0.5644 \cdot \frac{85}{9} \int_{\frac{49}{255}}^{\frac{21}{100}} \frac{d t}{t} \int_{\frac{1}{2}\left(\frac{49}{85}-t\right)}^{t} \frac{d u}{u} \int_{\frac{9}{85}}^{u} \frac{d v}{v}\right) \\
& \leq(0.187169+0.077309+0.019528) \eta x \log ^{-1} x \\
& =0.284006 \eta x \log ^{-1} x \text {. }
\end{aligned}
$$

Hence,

$$
\Omega_{1} \geq 0.958687 \eta x \log ^{-1} x .
$$

In the same way, it can be shown that

$\Omega_{2}+\Omega_{3}+\Omega_{4}+\Omega_{6}+\Omega_{8}+\Omega_{10}+\Omega_{12}+\Omega_{14}+\Omega_{17}+\Omega_{20}+\Omega_{23}$ 


$$
+\Omega_{26}+\Omega_{29}+\Omega_{32}+\Omega_{35}+\Omega_{38}+\Omega_{50}+\Omega_{54}+\Omega_{57}+\Omega_{60}
$$

$\geq \eta x \log ^{-1} x\left(\frac{85}{9} \int_{\frac{21}{100}}^{\frac{13}{60}} \frac{d t}{t} \int_{\frac{9}{85}}^{\frac{7}{20}-\frac{2}{3} t} \frac{1}{u} w\left(\frac{85}{9}(1-t-u)\right) d u\right.$

$-\frac{85}{9} \int_{\frac{21}{100}}^{\frac{13}{60}} \frac{d t}{t} \int_{\frac{9}{85}}^{\frac{7}{20}-\frac{2}{3} t} \frac{d u}{u} \int_{\frac{9}{85}}^{u} \frac{1}{v} w\left(\frac{85}{9}(1-t-u-v)\right) d v$

$+\frac{85}{9} \int_{\frac{13}{60}}^{\frac{12613}{49300}} \frac{d t}{t} \int_{\frac{9}{85}}^{\frac{29}{120}-\frac{t}{6}} \frac{1}{u} w\left(\frac{85}{9}(1-t-u)\right) d u$

$-\frac{85}{9} \int_{\frac{13}{60}}^{\frac{12613}{49300}} \frac{d t}{t} \int_{\frac{9}{85}}^{\frac{29}{120}-\frac{t}{6}} \frac{d u}{u} \int_{\frac{9}{85}}^{u} \frac{1}{v} w\left(\frac{85}{9}(1-t-u-v)\right) d v$

$+\frac{85}{9} \int_{\frac{12613}{49300}}^{\frac{3441}{9860}} \frac{d t}{t} \int_{\frac{9}{85}}^{\frac{897}{1972}-t} \frac{1}{u} w\left(\frac{85}{9}(1-t-u)\right) d u$

$-\frac{85}{9} \int_{\frac{12613}{49300}}^{\frac{3441}{9860}} \frac{d t}{t} \int_{\frac{9}{85}}^{\frac{897}{1972}-t} \frac{d u}{u} \int_{\frac{9}{85}}^{u} \frac{1}{v} w\left(\frac{85}{9}(1-t-u-v)\right) d v$

$+\frac{85}{9} \int_{\frac{297}{800}}^{\frac{653}{1700}} \frac{d t}{t} \int_{\frac{49}{100}-t}^{\frac{19}{160}} \frac{1}{u} w\left(\frac{85}{9}(1-t-u)\right) d u$

$-\frac{85}{9} \int_{\frac{297}{800}}^{\frac{653}{1700}} \frac{d t}{t} \int_{\frac{49}{100}-t}^{\frac{19}{160}} \frac{d u}{u} \int_{\frac{9}{85}}^{u} \frac{1}{v} w\left(\frac{85}{9}(1-t-u-v)\right) d v$

$+\frac{85}{9} \int_{\frac{653}{1700}}^{\frac{5073}{13120}} \frac{d t}{t} \int_{\frac{9}{85}}^{\frac{19}{160}} \frac{1}{u} w\left(\frac{85}{9}(1-t-u)\right) d u$

$-\frac{85}{9} \int_{\frac{653}{1700}}^{\frac{5073}{13120}} \frac{d t}{t} \int_{\frac{9}{85}}^{\frac{19}{160}} \frac{d u}{u} \int_{\frac{9}{85}}^{u} \frac{1}{v} w\left(\frac{85}{9}(1-t-u-v)\right) d v$

$+\frac{85}{9} \int_{\frac{5073}{13120}}^{\frac{5541}{13940}} \frac{d t}{t} \int_{\frac{9}{85}}^{\frac{133}{230}-\frac{82}{69} t} \frac{1}{u} w\left(\frac{85}{9}(1-t-u)\right) d u$ 


$$
\begin{aligned}
& -\frac{85}{9} \int_{\frac{5073}{13120}}^{\frac{5541}{13940}} \frac{d t}{t} \int_{\frac{9}{85}}^{\frac{133}{230}-\frac{82}{69} t} \frac{d u}{u} \int_{\frac{9}{85}}^{u} \frac{1}{v} w\left(\frac{85}{9}(1-t-u-v)\right) d v \\
& -0.000100) \\
& \geq \eta x \log ^{-1} x\left(0.5612 \cdot \frac{85}{9} \int_{\frac{21}{100}}^{\frac{13}{60}} \frac{d t}{t} \int_{\frac{9}{85}}^{\frac{7}{20}-\frac{2}{3} t} \frac{d u}{u}\right. \\
& -0.5644 \cdot \frac{85}{9} \int_{\frac{21}{100}}^{\frac{13}{60}} \frac{d t}{t} \int_{\frac{9}{85}}^{\frac{7}{20}-\frac{2}{3} t} \frac{d u}{u} \int_{\frac{9}{85}}^{u} \frac{d v}{v} \\
& +0.5612 \cdot \frac{85}{9} \int_{\frac{13}{60}}^{\frac{12613}{49300}} \frac{d t}{t} \int_{\frac{9}{85}}^{\frac{29}{120}-\frac{t}{6}} \frac{d u}{u} \\
& -0.5617 \cdot \frac{85}{9} \int_{\frac{13}{60}}^{\frac{12613}{49300}} \frac{d t}{t} \int_{\frac{9}{85}}^{\frac{1}{2}\left(\frac{49}{85}-t\right)} \frac{d u}{u} \int_{\frac{9}{85}}^{u} \frac{d v}{v} \\
& -0.5644 \cdot \frac{85}{9} \int_{\frac{13}{60}}^{\frac{12613}{49300}} \frac{d t}{t} \int_{\frac{1}{2}\left(\frac{49}{85}-t\right)}^{\frac{29}{120}-\frac{t}{6}} \frac{d u}{u} \int_{\frac{9}{85}}^{u} \frac{d v}{v} \\
& +0.5612 \cdot \frac{85}{9} \int_{\frac{12613}{49300}}^{\frac{3441}{9860}} \frac{d t}{t} \int_{\frac{9}{85}}^{\frac{897}{1972}-t} \frac{d u}{u} \\
& -0.5617 \cdot \frac{85}{9} \int_{\frac{12613}{49300}}^{\frac{1643}{4930}} \frac{d t}{t} \int_{\frac{9}{85}}^{\frac{1}{2}\left(\frac{49}{85}-t\right)} \frac{d u}{u} \int_{\frac{9}{85}}^{u} \frac{d v}{v} \\
& -0.5644 \cdot \frac{85}{9} \int_{\frac{12613}{49300}}^{\frac{1643}{4930}} \frac{d t}{t} \int_{\frac{1}{2}\left(\frac{49}{85}-t\right)}^{\frac{897}{1972}-t} \frac{d u}{u} \int_{\frac{9}{85}}^{u} \frac{d v}{v} \\
& -0.5644 \cdot \frac{85}{9} \int_{\frac{1643}{4930}}^{\frac{3441}{9860}} \frac{d t}{t} \int_{\frac{9}{85}}^{\frac{897}{1972}-t} \frac{d u}{u} \int_{\frac{9}{85}}^{u} \frac{d v}{v} \\
& +0.5612 \cdot \frac{85}{9} \int_{\frac{297}{800}}^{\frac{653}{1700}} \frac{d t}{t} \int_{\frac{49}{100}-t}^{\frac{19}{160}} \frac{d u}{u}
\end{aligned}
$$




$$
\begin{aligned}
& -0.5644 \cdot \frac{85}{9} \int_{\frac{297}{800}}^{\frac{653}{1700}} \frac{d t}{t} \int_{\frac{49}{100}-t}^{\frac{19}{160}} \frac{d u}{u} \int_{\frac{9}{85}}^{u} \frac{d v}{v} \\
& +0.5612 \cdot \frac{85}{9} \int_{\frac{653}{1700}}^{\frac{5073}{13120}} \frac{d t}{t} \int_{\frac{9}{85}}^{\frac{19}{160}} \frac{d u}{u} \\
& -0.5644 \cdot \frac{85}{9} \int_{\frac{653}{1700}}^{\frac{5073}{13120}} \frac{d t}{t} \int_{\frac{9}{85}}^{\frac{19}{160}} \frac{d u}{u} \int_{\frac{9}{85}}^{u} \frac{d v}{v} \\
& +0.5612 \cdot \frac{85}{9} \int_{\frac{5073}{13120}}^{\frac{5541}{13940}} \frac{d t}{t} \int_{\frac{9}{85}}^{\frac{133}{230}-\frac{82}{69} t} \frac{d u}{u} \\
& -0.5644 \cdot \frac{85}{9} \int_{\frac{5073}{13120}}^{\frac{5541}{13940}} \frac{d t}{t} \int_{\frac{9}{85}}^{\frac{133}{230}-\frac{82}{69} t} \frac{d u}{u} \int_{\frac{9}{85}}^{u} \frac{d v}{v} \\
& -0.000100) \\
& \geq(0.111673-0.037863+0.570625-0.100014 \\
& -0.085420+0.599832-0.063002-0.072362 \\
& -0.000822+0.010098-0.000782+0.004011 \\
& -0.000232+0.008592-0.000335-0.000100) \eta x \log ^{-1} x \\
& =0.943899 \eta x \log ^{-1} x \text {. }
\end{aligned}
$$

Hence,

$$
\Phi \geq 1.902585 \eta x \log ^{-1} x .
$$

The proof of Lemma 29 is complete.

10. The proof of the Theorem. From Lemmas 24-29, it follows that

$$
\Omega \geq 2.949096 \eta x \log ^{-1} x \text {. }
$$

Then using Lemmas 22 and 23, we obtain

$$
\pi(x+\eta x)-\pi(x)=S\left(\mathcal{A},(2 X)^{\frac{1}{2}}\right) \geq 0.011 \eta x \log ^{-1} x .
$$

Now (15) holds and the proof of the Theorem is complete.

\section{References}

[1] H. Cramér, On the order of magnitude of the difference between consecutive prime numbers, Acta Arith. 2 (1937), 23-46. 
[2] J.-M. Deshouillers and H. Iwaniec, Power mean values of the Riemann zetafunction, Mathematika 29 (1982), 202-212.

[3] G. Harman, Primes in short intervals, Math. Z. 180 (1982), 335-348.

[4] - On the distribution of ap modulo one, J. London Math. Soc. (2) 27 (1983), 9-18.

[5] D. R. Heath-Brown, Finding primes by sieve methods, Proc. 1982 ICM, Warsaw, 1983, Vol. 1, PWN-Polish Sci. Publ., Warszawa, 1984, 487-492.

[6] D. R. Heath-Brown and H. Iwaniec, On the difference between consecutive primes, Invent. Math. 55 (1979), 49-69.

[7] M. N. Huxley, On the difference between consecutive primes, ibid. 15 (1972), $164-170$.

[8] H. Iwaniec, A new form of the error term in the linear sieve, Acta Arith. 37 (1980), 307-320.

[9] H. Iwaniec and J. Pintz, Primes in short intervals, Monatsh. Math. 98 (1984), $115-143$.

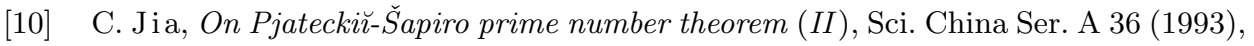
913-926.

[11] - Goldbach numbers in short interval, Sci. China Ser. A 24 (1994), 1233-1259 (in Chinese); I. Sci. China Ser. A 38 (1995), 385-406; II. Sci. China Ser. A 38 (1995), $513-523$.

[12] - On the difference between consecutive primes, Sci. China Ser. A 25 (1995), 785-804 (in Chinese); Sci. China Ser. A 38 (1995), 1163-1186.

[13] - On the exceptional set of Goldbach numbers in the short interval, Acta Arith., to appear.

[14] H. Li, On the Goldbach numbers in short interval, Sci. China Ser. A 38 (1995), 641-652.

[15] - Primes in short intervals, preprint.

[16] H. L. Montgomery, Topics in Multiplicative Number Theory, Lecture Notes in Math. 227, Springer, Berlin, 1971.

[17] Chengdong P an and Chengbiao P an, Goldbach Conjecture, Science Press, Beijing, 1981 (in Chinese).

[18] - - - The Basis of Analytic Number Theory, Science Press, Beijing, 1991 (in Chinese).

[19] A. Selberg, On the normal density of primes in short intervals, and the difference between consecutive primes, Arch. Math. Naturvid. 47 (1943), 87-105.

[20] N. Watt, Short intervals almost all containing primes, Acta Arith. 72 (1995), 131167.

[21] - Kloosterman sums and a mean value for Dirichlet polynomials, J. Number Theory, to appear.

Institute of Mathematics

Academia Sinica

Beijing, 100080, China 